\title{
Abstracts of the XXXI Annual Meeting of The Association of European Paediatric Cardiologists, Göteborg, 15-18 May 1996
}

I-01

The aorta after arterial switch operation

Hutter PA, de Bruyn D, Harinck E, Meijboom EJ

Department of Cardiology, Wilhelmina Children's Hospital, University of Utrecht, Utrecht, The Netherlands

Concerns have been voiced that the pulmonary valve serving as the neoaortic valve after arterial switch operation (ASO) is susceptible to dilatation and incompetence. Failure of the anastomosis to grow might result in stenosis. This study determines the incidence of aortic complications after ASO in a group of patients operated on since 1977. Ninetyseven patients still alive and in the Netherlands formed the study group. Median follow-up is 7.9 (range 0.4 to 17.4) yrs. Thirty-four patients had associated VSD, eight patients had aortic coarctation and one had interrupted aortic arch. A normal aortic root diameter was found in 77 patients; $75 / 77$ had no or only trivial aortic incompetence; $2 / 77$ had mild (grade II/IV) incompetence. An aortic root diameter above normal for body surface area was found in 20 patients. No or only trivial incompetence was seen in $17 / 20$ patients, two patients have mild incompetence and one patient developed moderate (grade II/VI) incompetence with clinical significance 1.5 yrs after ASO. Normal aortic flow velocities were found in 93/97 patients. Increased flow has been observed in 4/97. One patient was operated for subaortic stenosis which was underestimated preoperatively and one patient with transposition, VSD and interrupted aortic arch required reoperation one week after ASO for stenosis at the cannulation site. Two patients have aortic stenosis at the site of the anastomosis; one of these had patch enlargement of the anastomosis during reoperation for pulmonary stenosis, the other patient did not require reoperation to date. Recoarctation was excluded in 5/8 patients. Balloon angioplasty for recoarctation was necessary in two patients, including the one patient with interrupted aortic arch. One patients awaits reintervention. We conclude that after ASO, the neoaortic valve is larger than normal in $20 \%$ of patients and mild or moderate aortic regurgitation is seen in $5 \%$. A clear relationship between dilatation and insufficiency could not be demonstrated. Stenosis of the aortic anastomosis was seen in $2 \%$ of the patients.
I-02

Pulmonary function in children after palliative Mustard/Senning correction of transposition of the great arteries with ventricular septal defect and pulmonary hypertension

Sulc J, Hruda J, Zapletal A, Hucin B, Samanek M

Kardiocentrum, University Hospital Motol, Prague, Czech Republic

Atrial repair of TGA is known to be associated with several long-term problems, including pulmonary function (PFT) impairment: in $88 \%$ of patients operated on at a late age ( $4.4 \mathrm{yrs})$ and $74 \%$ of those operated earlier ( $0.8 \mathrm{yrs})$ for TGA with or without VSD, PFT was abnormal (Samanek 1989, Sulc 1995). The most prominent abnormality was stiff lung. Rare patients after palliative Senning/Mustard repair (i.e., VSD could not be closed because of severe $\mathrm{PH}$ ) continue to have $\mathrm{PH}$ postoperatively. Five patients were followed $7.7 \pm 2.6$ (mean \pm SD, median 6.2) yrs after palliative atrial repair. They were operated on at 2.6 2.3 (median 1.6) yrs. Staticlung volumes (by bodyplethysmography), airway patency indices flow-volume curve and bodyplethysmography) and lung elasticity (quasi-static pressure-volume curve) were measured. Mean values of lung recoil pressure at three levels of total lung capacity (TLC) were increased, i.e. $132 \%, 127 \%$ and $126 \%$ of predicted value. Mild increase of the ratio of residual volume to TLC ( $119 \%$ of predicted value) indicating hyperinflation was found. Central airway obstruction indicated by a reduced specific airway conductance (to $65 \%$ of predicted value) was found. Neither peripheral airway obstruction nor lung volume restriction was found. Nevertheless, at least one PFT abnormality was found in all our subjects. We conclude that while all our patients after palliative Senning/Mustard repair reveal PFT abnormalities, surprisingly, this impairment is not more severe than in those after conventional atrial procedure. 
I-03

The pulmonary arteries and the pulmonary vascular bed following total cavopulmonary connection

Buheitel $G$, Hofbeck $M$, Singer $H$, van dem Emde J

Departments of Pediatric Cardiology and Cardiac Surgery, University of Erlangen, Erlangen, Germany

The aim of this study was to assess the development of the pulmonary arteries (PA) in the medium-term follow-up after TCPC. For this purpose the angiographies of 25 patients were evaluated (mean interval $40.8 \pm 12.8$ months after TCPC). The patients were divided into two groups depending on their preoperative Qp:Qs relation. In the following Table the size of the pulmonary arteries was expressed as the number of standard deviations from normal values (z-score) related to the body surface area (BSA).

\begin{tabular}{|c|c|c|c|c|}
\hline \multirow[b]{3}{*}{ Months postop } & \multicolumn{2}{|c|}{ Qp:Qss 1} & \multicolumn{2}{|c|}{ Qp:Qs>1 } \\
\hline & Preop & Postop & Preop & Postop \\
\hline & & $42.8 \pm 14.3$ & & $39.0 \pm 11.4$ \\
\hline Left PA ( $\mathrm{z})$ & $-4.3 \pm 7.8$ & $-6.0 \pm 4.7$ & $9.2 \pm 13.4$ & $0.7 \pm 9.1$ \\
\hline Right PA (z) & $0.1 \pm 7.5$ & $-0.9 \pm 6.8$ & $8.2 \pm 10.9$ & $3.1 \pm 7.7$ \\
\hline $\mathrm{Rpl} \mathrm{U} \cdot \mathrm{m}^{2}$ & $1.8 \pm 0.8$ & $1.6 \pm 0.7$ & $1.2 \pm 0.5$ & $1.6 \pm 0.8$ \\
\hline
\end{tabular}

Significant differences were noted between the large preoperative and the smaller postoperative diameters of both PAs in the group with an increased pulmonary blood flow prior to the TCPC. The preoperative PA-size expressed as McGoon-ratio and Nakakta-index was also large in the Qp:Qs > 1 group with a significant decrease 39 months after TCPC. There were no correlations between the postoperative pulmonary artery size and the postoperative cardiac index, the pulmonary vascular resistance or the pulmonary artery pressure. In conclusion, in patients with Qp:Qs>1 prior to the TCPC, the size of the pulmonary arteries is large and decreases towards a normal diameter in the medium-term followup. In patients with Qp:Qs $\leq 1$ prior to surgery, the small pulmonary arteries remain at the same sub-normal size related to BSA. In both groups the pulmonary vascular resistance showed no significant change.

I-04

Pulmonary atresia and intact ventricular septum-a retrospective study of 23 children

Sunnegairdh J, Joelsson B-M, Berggren $H$, Südow G, Mellander $M$, Mellgren $G$

Pediatric Cardiology, Östra Sjukhuset, Göteborg, Sweden

In PA-IVS there is a number of associated abnormalities; even though these different abnormalities may be perfectly diagnosed, the decision of surgical treatment directed towards a uni- or biventricular heart may be impossible to take in the neonatal period. Since 1980, 23 children (11 girls, 12 boys) with PA-IVS were operated in our institution. The initial evaluation of the right ventricle was performed by angiography and echocardiography in 12 patients and by only echocardiography in 11 patients. In 22/23 patients the right ventricle was assessed to be smaller than normal and coronary abnormalities were found in 7 patients. Twenty-one patients had an initial aortopulmonary shunt operation combined with an outflow correction in 12 . In 8 cases an additional right ventricular outflow correction was performed later. In 1 child a pulmonary valvotomy was performed as the only initial surgical treatment, and one child with RV coronary fistulas survived because of a delayed closure of the duct with later shunt operations, $R V$-outflow correction and even a total cavopulmonary connection (TCPC). At present 7 children have a two-chamber and 8 have a one-chamber system (TCPC 6, bidirectional Glenn 2). The overall mortality is $26 \%$ and among the children with coronary abnormalities $50 \%$. Severe coronary abnormalities were diagnosed at autopsy in one child without previous angiographic evaluation. Four of the children with coronary abnormalities had a TCPC with three surviving the operation. One child died suddenly at home while all other were hospital deaths at time of surgery. The overall mortality and the proportion of uni- and biventricular systems in the survivors were in agreement with the results reported from other centers.
I-05

Surgical valvotomy of aortic valve stenosis-long-term outcome Gildein HP, Kleinert S, Pawade A, Karl T, Wilkinson JL Departments of Cardiology and Cardiac Surgery, Royal Children's Hospital, Melbourne, Australia

Early results of surgical valvotomy for aortic valve stenosis are excellent. However, in the long term, reintervention-which may involve aortic valve replacement-will be required in a considerable number of cases. We investigated the long-term outcome of patients who were older than 1 year at initial surgery and had a follow-up period of at least 6 years. From 1978 until 1987 surgical valvotomy of the aortic valve was performed in 56 consecutive patients at ages ranging from 1 to 17.5 years (mean 9.5-5.0 years). The aortic valve was bicuspid in 48 patients, tricuspid in 8 and thickened in 11 . There were no hospital deaths. Late death occurred in 2 patients, both of them unrelated to cardiac causes. Four patients were lost to follow-up. In 12 of the remaining 52 patients (23\%), reintervention (revalvotomy in 4, aortic valve replacement in 4 ( 2 homograft, $1 \mathrm{St}$. Jude valve, 1 bioprosthesis), Ross procedure in 1 , and balloon dilatation in 4) was performed $8 \pm 2.5$ years after the first procedure without mortality. The follow-up time for the patients without reintervention was $11 \pm 3$ years. None of them is currently on medication, and only 1 patient, who refuses further treatment, is symptomatic on exertion. Surgical valvotomy for aortic valve stenosis has produced excellent results with little morbidity and mortality. The rate of reintervention remains low during childhood and adolescence.

I-06

Postoperative results in isolated subaortic stenosis

Kaneva A, Tzonsarova M, Pilossoff V, Todorov A, Pavlova $M$

National Center for Cardiovascular Diseases, Sofia, Bulgaria

The aim of the study was to assess retrospectively the results of surgical treatment in patients with isolated subaortic stenosis (ISAS). Evaluation was made on the basis of clinical condition, NYHA functional class, ECG, ECHO CG-peak gradient (PG), in the left ventricular outflow tract (LVOT), aortic insufficiency (AI) and morphological characteristics of LVOT. Between January 1985 and July 1995, 110 pts with subaortic stenosis were operated on and 39 of them had ISAS (27 males, 12 females); mean age at operation $13 \pm 7.8$ yrs (range $4.5-45$ ); PG $88 \pm 28$ $\mathrm{mm} \mathrm{Hg}$ (range 40-155); trivial AI 88\%. Twenty-nine had fibrous, 9 with fibro-muscular and 1 with tunnel-type of ISAS. Operative procedures included incision of ISAS in 20 pts and blunt dissection in 19. Morrow myectomy was performed in $28 \mathrm{pts}$ and removal of the fibrotic extensions towards the aortic and/or mitral valve in 12 pts. Aortic valve prosthesis was implanted in $1 \mathrm{pt} \mathrm{having} \mathrm{infective} \mathrm{endocarditis.} \mathrm{Pts} \mathrm{were}$ followed $3.2 \pm 1.8$ yrs (range $0.6-9$ ). There were no operative or early deaths. Onept died 6 mo postoperatively due to prosthetic endocarditis. Thirty-two pts (88\%) showed favorable results: PG 0 to $20 \mathrm{~mm} \mathrm{Hg}, \mathrm{AI}$ none or trivial. The remnants of tissue removed were found by ECHO in 16 pts ( 13 after excision and 3 after blunt dissection). The postoperative results were unsatisfactory in 6 pts. In conclusion, 1) during the midterm follow-up, postoperative result in ISAS are favorable in $88 \%$ of cases; 2) unsatisfactory results are connected mainly with type of ISAS (tunnel), concomitant narrow ring, complications (infective endocarditis) and iatrogenical injury; 3) ECHO CG shows tissue remnants in LVOT that do not cause significant hemodynamic disturbances; and 4) patients operated from ISAS need close and regular control. 
I-07

Quality of life after bidirectional cavopulmonary anastomosis

Hruda J, Sulc J, Bartakova H, Hucin B, Kostelka M, Tlaskal T, Reich O, Skovranek J, Horvath P

Kardiocentrum, Untversity Hospital Motol, Prague, Czech Republic

As bidirectional cavopulmonary anastomosis (BCA) is viewed as a temporary palliation, its long-term effects are not yet defined. During $1987-95$, BCA was performed in 68 pts. Six $(8.8 \%)$ pts died in hospital, $24(35.3 \%)$ pts underwent further surgery ( 4 died) and 38 pts had no other surgery (1 death, overall mortality $16.2 \%$ ). Sixty-two pts after BCA, performed at the age $5.39 \pm 4.97(0.42-25.2) \mathrm{yrs}$, were followed for $2.55 \pm 1.77(0.12-7.84)$ yrs. Hemoglobin, $\mathrm{SO}_{2}$, echocardiography, radionuclide angiography and lung function testing were employed. Functionally, 18 were classified as NYHA I (29\%), 36 class II (58\%) and 8 class III (13\%). Forty-eight pts $(77.4 \%)$ received medication, 4 of them antiarrhythmics. At latest follow-up, 7 pts had no cyanosis (11.3\%), bur 27 had mild (43.5\%), 21 moderate (33.9\%) and 7 severe cyanosis (11.3\%). Hemoglobin level $(\mathrm{g} / \mathrm{l})$ decreased from $182.3 \pm 33.1$ to $135.3 \pm 17.5$ postoperatively ( $\mathrm{p}=0.0001$ ), but at the last follow-up rose again to $165 \pm 24.3$ ( $\mathrm{p}=0.0001$ ). $\mathrm{SO}_{2}$ however did not drop markedly: $69.8 \pm 10.3,80.1 \pm 10.2(p=0.0001)$ and $78.9 \pm 9.2 \%(p=0.499)$, respectively. The body weight increased from a $Z$-value of -1.79 (SD 1.17) by $0.62(p=0.007)$, the height from -1.13 (SD 1.5) by only $0.14(p=0.618)$. Echocardiography demonstrated minimal pressure gradient over BCA in 5 pts (8\%), ventricular systolic dysfunction in 5 pts $(8 \%)$ and significant $A V$ valve regurgitation in 11 pts (17.7\%). Twenty pts with BCA and competitive pulmonary blood fow (PBF) were studied by radionuclide angiography; cavocaval collaterals developed in $8(40 \%)$ pts. PBF was accomplished by BCA in $46.3 \%$ of pts, while $53.7 \%$ by other sources. Lung function testing was completed in 18 pts. Sixteen of them $(89 \%)$ revealed lung function abnormality, mostly hyperinflation $(56 \%)$ and restriction (50\%). BCA represents an effective short-term palliation, but quality of life deteriorates with time in majority of pts.

\section{II-01}

How to manage the infant with critical aortic stenosis when first line palliations fail?

Solymar L, Südow G, Berggren H, Eriksson BO, Holmgren D From the Department of Pediatrics and Thoracic Surgery, University of Göteborg, Göteborg, Sweden

Critical aortic stenosis in infancy is associated with high morbidity and mortality and requires urgent intervention for patient survival. If the size of the left ventricle is adequate for biventricular repair, aortic valvar dilation or valvotomy will, in the majority of cases, effectively reduce the left ventricular outflow stenosis. However, some patients remain in severe heart failure due to persistent left ventricular outflow obstruction and/or postoperative aortic insufficiency or develop other complications of the primary palliation, making further corrective surgery mandatory. Eight patients, all presenting within the first days of life and ductdependent for adequate systemic circulation were primarily palliated with transventricular dilation and/or valvotomy and/or balloon dilation as a single operation (4) or as repeated procedures using various combinations of these palliations (3). Three remained in severe heart failure, 3 suffered valve avulsion, and 1 had a persistent severe and increasing gradient. The aortic valve was replaced with the patient's own pulmonary valve (Ross-op) in 6 and with homograft in 1; the latter underwent Ross-op at the age of 1.5 yrs because of conduit dysfunction. Seven pts (88\%) survived surgery; 1 patient could not be weaned off bypass. He was found to have severe endocardial fibroelastosis at autopsy. One late death occured due to hypertrophic cardiomyopathy, and PHT one year postoperatively. The rest had steady clinical improvement and are doing well and off any medication. Apart from trivial regurgitation in three, the pulmonary autografts are performing well and seem to grow with the patients. In conclusion, the Ross operation offers successful solution in infants with critical aortic stenosis in whom other palliations fail.
Notes 


\section{II-02}

Incidence and management of pulmonary venous obstruction after repair of total anomalous pulmonary venous connection Stimper O, Wright JGC, Sethia B, de Giovanni JV, Silove ED, Brawn WJ The Children's Hospital, Birmingham, Untted Kingdom

Pulmonary venous obstruction (PVO) after repair of total anomalous pulmonary venous connection (TAPVC) is commonly regarded to be a lethal lesion. We present our experience with TAPVC repair, the incidence, the management and outcome of PVO over a 5-year period (1988-1993). Forty-seven consecutive patients with TAPVC underwent repair. Mean age was 3.7 months ( 1 day- 6.3 yrs). Twenty-three pts had supracardiac TAPVC (49\%), 9 cardiac (19\%), 9 infracardiac (19\%) and 6 mixed TAPVC (13\%). Venous return was obstructed in 22 $(47 \%)$. There were 3 early deaths $(6.4 \%)$, two of which were cardiac. Mean hospital stay was 8.2 days (range 5-28 d). At a mean follow-up $(\mathrm{F} / \mathrm{U})$ of 3.7 years $(1.4-6 \mathrm{yrs}) 39$ pts are alive and well and on no medication $(83 \%)$. None of these pts has clinical or ultrasound evidence of pulmonary hypertension or pulmonary venous obstruction. PVO developed in 5 patients $(10.6 \%$ ) at a mean $\mathrm{F} / \mathrm{U}$ of 12 weeks (range 1 day8 months). One further pt with PVO was referred from abroad. A total of 12 reoperations were performed, with one operative death. Surgical techniques included: (1) revision of anastomoses, (2) excision of stenosed venous segments and reanastomosis, (3) creation of an ASD. A total of twelve angioplasty procedures were performed at different intervals. Four stents were implanted with only 1 medium-term patency. There was one late non-cardiac death. Autopsy excluded PVO or pulmonary hypertensive changes. Four pts are alive $2-4.3$ years (mean $2.9 \mathrm{yrs}$ ) after initial reoperation. Only 1 patient has RV pressures of more than $60 \%$ of systemic pressures. PVO after TAPVC repair responds to an aggressive joint surgical and interventional approach. The results obtained are promising with a medium-term survival of $67 \%$ of patients who develop postoperative PVO and $89 \%$ of all pts presenting with TAPVC.

\section{II-03}

The multitrack angiography catheter-a new tool for complex catheterization in pediatric cardiology

Bonhoeffer P, Piéchaud JF, Stümper O, Bonnet D, Aggoun Y, Sidi D, Kachaner J

Hôpital Necker, Enfants-Malades, Paris, France

A new catheter system that facilitates angiography and pressure measurements during complex cardiaccatheterization procedures in paediatric cardiology was developed and evaluated in a series of 84 patients. Ages were 1 day-20 years and weights between 2.1 and $80 \mathrm{~kg}$. The MultiTrack Angio catheter system is a single lumen side-hole catheter with a short distal extension which contains a lumen for a standard guidewire. The catheter is introduced over a previously placed guidewire running through this distal extension. It can then be manipulated within the heart by sliding along the guidewire. The tip of the catheter is always stabilized by the guidewire, which allows for enhanced angiography and pressure recordings. In 31 patients diagnostic procedures were performed and in 53 patients interventional procedures. The decision to use the Multi-Track Angio catheter system were based on three criteria: unsatisfactory angiography obtained with conventional equipment, difficult catheter course requiring use of a flexible guidewire, and requirement for repeated angiography and pressure recordings during interventional procedures. High quality angiography could be performed in all cases without catheter recoil. Recordings of pull-back pressure traces were enhanced due to controlled catheter movements across stenotic lesions. In conclusion, the Multi-Track Angio catheter system allows for high quality angiography and pressure recordings during both diagnostic and interventional catheterization. The main advantage of the system is that both angiography and pressure recordings can be performed repeatedly from stable catheter positions using a previously placed guidewire. This largely decreases the need for guidewire manipulations or catheter exchanges. This reduces procedure time, potential risk of complications and facilitates complex interventions.
II-04

Intracardiac stents solve a baffling problem

Bu'Lock FA, Tometzki AJ, Kitchener DJ, Arnold R, Peart I, Walsh KT Department of Cardiology, Alder Hey Children's Hospital, Liverpool, UK

Systemic venous pathway obstruction following intraatrial baffle repair for transposition of the great arteries (TGA) may be associated with effort limitation and sudden death. Balloon angioplasty has had only limited success, with a significant restenosis rate. Of 154 long-term survivors of Mustard type repair of TGA, 33 have undergone cardiac catheterization since January 1993. In 18 of these, angiographic narrowings of superior, inferior or both limbs of the systemic venous pathways were treated by stent implantation. Ten had previously undergone balloon angioplasty for baffle stenosis, and $1 \mathrm{pt}$ had recently been successfully resuscitated from ventricular fibrillation. Twenty-four Johnson \& Johnson stents (22 P308s, 1 P188, 1 P5015) were deployed during 19 procedures, 16 in the inferior systemic baffle and 6 in the superior limb. In 2 pts, stents were deployed in the right iliac vein following malposition and intravascular retrieval (the only complications). Pressure gradients were reduced from mean 5 (range 3-11) to $1(0-2) \mathrm{mm} \mathrm{Hg}$ and $3(0-4)$ to $<1(0-3) \mathrm{mm} \mathrm{Hg}$ in the superior and inferior limbs respectively. However, severity of stenosis was most clearly assessed from the angiogram. Minimum parhway diameter was increased from 8.7 (3.5-14.1) to 13.7 (8.7-18.5) $\mathrm{mm}$ in the superior limb and from $9.6(4.5-16.4)$ to 15.7 (11.9-21.8) $\mathrm{mm}$ in the inferior limb following stenting. Most patients reported a subjective improvement in effort tolerance following the procedure. In 3 patients, right ventricular failure has progressed; 2 have died ( 1 post transplant) and 1 awaits transplant. Three pts had electrophysiological studies and radiofrequency ablation of atrial arhythmias at the time of stenting, 2 pts have required dual chamber pacemakers for sinus node dysfunction and 2 require maintenance amiodarone. Nine pts are taking ACE inhibitors. Stenting of systemic venous pathway obstruction following Mustard repair of TGA is safe and is probably more effective than balloon angioplasty alone.

II-05

Transcatheter occlusion of the atrial fenestration after the Fontan operation

Rosenthal E, Qureshi SA, Anderson D, Baker EJ, Tynan M

Department of Paediatric Cardiology, Guy's Hospital, London, United Kingdom

The fenestrated Fontan operation has reduced the incidence of low output states following the Fontan operation. In some centres the fenestration is closed in the early perioperative period while in others it is not closed at all. We have reviewed our experience with transcatheter closure of the atrial fenestration after a fenestrated Fontan operation. Transcatheter occlusion of a $4 \mathrm{~mm}$ atrial fenestration was attempted in 20 children aged 3.6 to 16.3 (median 9.5) years and 1 adult aged 47 years. The time from surgery to catheterisation ranged from 5-39 months (median 18 months). Catheterisation was performed under general anaesthesia and aided by transesophageal echocardiography in all. In 4 patients (pts) the fenestration was either occluded or so small that only a guidewire could cross it. The time to the catheterisation in these 4 children was 8-19 months (mean 16 months) and their oxygen saturations were $97-99 \%$. In 17 patients the fenestration was patent at catheterisation, 5-39 months (median 16 months) after surgery (oxygen saturations of $85-98 \%$ ). A $17 \mathrm{~mm}$ Rashkind umbrella was implanted in 15 pts and a $12 \mathrm{~mm}$ umbrella in 2 pts. In 5 pts the umbrella was front loaded into a 7 or 9 Fr sheath. Saturations rose to $91-99 \%$ at the end of the procedure. Complications include $1 \mathrm{pt}$ who had SVT that required a DC shock and $1 \mathrm{pt}$ who had a transient nodal rhythm. Patients were heparinized for 24 hours after the procedure following which aspirin was administered for 2 months except for 3 pts who were already anticoagulated. In conclusion, spontaneous occlusion of a $4 \mathrm{~mm}$ fenestration after the fenestrated Fontan operation occurs in a minority of patients. Transcatheter occlusion is safe and effective even when delayed beyond the early postoperative period. 
II-06

Atrial septal defect repair by the buttoned device placed over a wire-early follow-up results and comparison to direct placement Onorato E, Berger F, Rey C, Haddad G, Lang P, Walsh KP, Worms A-M, Rao PS, Sideris EB San Donato Milanese Hospital, Milan, Italy

To evaluate the results of atrial septal defect (ASD) occlusion by the overthe-wire placement (OW) of the buttoned device (DV), we compared the acute and early follow-up results (up to 1 year) with those of direct placement (DP). We compared 117 OW and 104 DP consecutively performed cases.

$\begin{array}{lcccccc} & \mathrm{N} & \text { ASD }_{(\mathrm{mm})} & \text { DV }_{(\mathrm{mm})} & \text { FO (\%) } & \text { ABAND (\%) } & \text { COMPI } \\ \text { DP } & 104 & 6-26 & 25-30 & 76 & 10 & 4 \\ \text { OW } & 117 & 9-31 & 25-60 & 82 & 5 & 1\end{array}$

In $10 \%$ of attempted OW cases with eccentric ASDs, the wire was withdrawn and DP was required. The range of ASD repair increased to $31 \mathrm{~mm}$ and the abandoned cases (ABAND) decreased. There were 4 acute complications with DP ( 1 atrial perforation, 1 unbuttoning, 1 embolization) in comparison to only one unbuttoning with $\mathrm{OW}$. On follow-up two OW cases required a second device (1) or surgery (1) for residual shunts in comparison with $3 \mathrm{DP}$ cases $(2 \mathrm{small}$ mitral perforations, 1 residual shunt). The OW method offers better alignment and centering along with better stability of the device and it is less operator dependent. Because of the better stability, larger DVs up to $60 \mathrm{~mm}$ were used, increasing the range of the method. OW manipulation is possible avoiding injury of critical structures and of the atrial wall.

III-01

Transcatheter occlusion of the patent ductus arteriosus with cook detachable coils

Tometzki AJP, Walsh KP, Arnold R, Peart I, Bu'lock FA, Sreeram N, Abdulhamed JM, Godman MJ

Royal Liverpool Children's Hospital, Liverpool, Burmingham Children's Hospital, Birmingham, Royal Hospital for Stck Children, Edinburgh, United Kingdom and Prince Sultan Cardiac Centre, Riyadh, Saudi Arabia

Seventy-one consecutive patients, aged 1.2-22 years, with a PDA underwent elective transcatheter closure with a new Cook detachable coil system. Forty-five were native PDAs with a minimum diameter of $1.0-5.0 \mathrm{~mm}$ (median $2.0 \mathrm{~mm}$ ). A further 26 had residual leaks following previous occlusion procedures. A transvenous approach was used in the majority of patients with a 4-6.3 Fr delivery catheter. A transarterial route was solely used in 12 patients. A total of 133 detachable Cook coils were successfully implanted in 70 patients. Each received between 1 and 7 coils to produce a satisfactory angiographic result. In one child, with a residual PDA, the lesion could not be crossed by a $4 \mathrm{Fr}$ catheter and the procedure was abandoned. Complete occlusion of native PDAs, assessed by color flow echocardiography, was achieved in 40/45 (89\%) at 24 hours, $41 / 45(91 \%)$ at 1 month and $43 / 45(96 \%)$ by 6 months follow-up. One embolization, of a $5 \mathrm{~mm}$ coil, occurred. Eight coils were electively removed due to poor positioning. One child has undergone a second coil procedure, for a residual leak, resulting in complete occlusion. In the residual PDA subset, twenty six $89 \%$ were occluded at 24 hours and 25/26 (96\%) at one month post-implantation. Left pulmonary artery velocity was increased post-implantation in two patients in thisgroup. Transcatheter occlusion using detachable Cook coils is a safe, effective and cost-efficient alternative to presently available devices. The delivery system benefits from being fully retrievable until a satisfactory position is obtained.
Notes 


\section{III-02}

Interventional ductal closure in low weight infants

Neuss MB, Lê TP, Grabitz RG, Hagel KJ, Redel DA

Department of Pediatric Cardiology, University of Bonn, Bonn, Division of Pediatric Cardiology, University of Gießen, Gießen and Department of Pediatric Cardiology, University of Aachen, Aachen, Germany

Several interventional tools are now available for interventional closure of the PDA, such as foam plugs, umbrellas and coils. In most cases the diameter of the implantation catheter is too big $(>6 \mathrm{Fr}$ ) for application in children less than $5 \mathrm{~kg}$. First we introduced an experimental study in newborn piglets $(n=13)$, weighing 1.2 to $2.3 \mathrm{~kg}$ (mean $2.0 \mathrm{~kg}$ ). Divided Palmaz-Schatz coronary stents (Johnson \& Johnson) were transvenously delivered in the arterial duct to maintain vessel patency. Two to six days later retrievable coils (0.018"-0.028", Duct-Occlud, pfm, Cologne), dumb-bell or double-disk shaped, were inserted into the stented duct via 3 Fr or 4 Fr catheters by venous approach. The retrievability of the coils allows exact and safe coil placement. We could demonstrate, that depending on the coil configuration and size, either flow reduction or complete occlusion could be achieved. Between August 1992 and October 1995, seven low weight infants $(1.3-5 \mathrm{~kg})$ with an age of 6 weeks to 3 months were treated with this method. Complete occlusion could be demonstrated in 4 patients. One received an additional coil after one year for closure of a residual shunt. One tiny residual shunt is still patent ( 4 weeks follow-up). In two patients a large elastic duct was closed by elective surgery after coil retrieval. Our initial results showed, that retrievable coils are a new interventional tool for interventional PDA closure in children with a weight less than $5 \mathrm{~kg}$.

\section{III-03}

Problems encountered during introduction of Gianturco coils for transcatheter occlusion of patent ductus arteriosus Galal $O$, deMoor $M$, Fadley $F$, Naffa $S$, Oufi $S$ Department of Cardiovascular Diseases, King Faisal Specialist Hospital and Research Center, Riyadh, Saudi Arabia

To present the problems encountered during the introduction of transcatheter occlusion of the patent ductus arteriosus using Gianturco coils and to report the short-term follow-up data. Between January 1994 and August 1995 a total of 72 pts underwent cardiac catheterization for occlusion of their PDA using a Gianturco coil. Forty-four pts (70\%) were done on an outpatient basis. Usually anterograde coil occlusion was accomplished except with 6 cases in which the ductus could only be passed retrogradely. In 64 of the 72 patients $(88 \%)$ the coils were implanted successfully. In $44 \mathrm{pts}(70 \%)$ a single coil was deployed while 20 pts required multiple coils (2-7). Eight children were sent for elective surgery when coil occlusion was not possible or had failed. In 2 cases coil occlusion was not possible. In 6 cases the procedure was concluded after failure to implant the coil(s). Forty-three of 64 pts (67.2\%) had total occlusion as judged by angiography ten minutes after implantation of the coil(s). A further 15/64 (21.9\%) more patients had occlusion as judged by color Doppler before discharge. Thus total occlusion rate at discharge was $89.6 \%$. In 14 pts embolization of the coil occurred; the coil was retrieved in all but one, which was left in the periphery of the pulmonary artery. In eleven of these patients, another coil(s) was (were) used for successful closure of the PDA. Five pts had pull through of the coil. The median fluoroscopy time was 12.5 (range 4-89; mean 18) $\mathrm{min}$. The longest fluoroscopy time was in those with embolization. In conclusion, transcatheter occlusion of the PDA using the Gianturco coil is an effective, relatively safe and an inexpensive technique. In the learning curve there seems to be a relatively high embolization rate. These complications should be considered as minor problems since the coil(s) retrieval can generally be accomplished quickly and safely.
III-04

Safety and efficacy of transarterial occlusion of the patent ductus using Gianturco coils

Radtke WAK

South Carolina Children's Heart Center, Charleston, USA

Forty-six consecutive patients ( $70 \%$ female) were catheterized with the intention to perform transvenous PDA closure using Gianturco coils. Five of these patients had a residual PDA after previous surgical PDA ligation. Forty-two patients, aged 10 months-15.1 years (median 3.4 yrs) with body weights of $5.3-52.1 \mathrm{~kg}$ (median $13.2 \mathrm{~kg}$ ) underwent implantation of 1 to 3 coils. Two patients underwent additional interventions during the same catheterization. In 4 patients coil implantation was not attempted because of duct diameter of more than $4.5 \mathrm{~mm}(2 \mathrm{pts})$ or because additional lesions required surgical repair ( $2 \mathrm{pts})$. Under ketamine sedation implantation was performed from the aortic side in standard technique with a 4 or $5 \mathrm{Fr}$ catheter. If a residual shunt was present additional coils were implanted during the same session (7 pts). A third coil was placed from transvenous approach. The coil diameter was chosen to be 1.7-2.0 times the smallest ductus diameter, but small enough to fit into the aortic ductus ampulla. If protrusion into aorta or pulmonary artery was significant, the coil was removed and a new coil implanted ( $2 \mathrm{pts}$ ). Coil sizes ranged from $3 \mathrm{~mm} \times 2 \mathrm{~cm}$ to $8 \mathrm{~mm} \times 13$ $\mathrm{cm}$. Smallest duct diameters were $0.4-4.3 \mathrm{~mm}$ (mean $1.5 \mathrm{~mm}$ ). All attempred implantations were successful. All coils were properly positioned. Total fluoroscopy times were 6-34 min (mean $18 \mathrm{~min}$ ). Twentyseven procedures were performed as outpatients. Fourteen patients were discharged the next morning. Closure was complete in $81 \%$ after 10 min; in $95 \%$ after 12 hours and in $96 \%$ after 6 months. No embolization and no complications occurred. One early partial recanalization was observed. There were no residual ductus murmurs. No stenosis of distal aortic arch or left pulmonary artery was found. Cost of the implanted coil is US\$34.20. Transarterial coil occlusion using Gianturco coils is safe, clinically effective and cost-effective.

III-05

Infant buttoned device for the occlusion of patent ductus arteriosus - early clinical experience

Sideris EB, Rey C, De Lezo JS, Solymar L

Athenian Institute of Pediatric Cardiology, Athens, Greece

The purpose of this study was to assess the efficacy and safery of a modification of the buttoned device, the infant buttoned device (IBD), capable of occluding ducts (PDAs) of all ages including infants. IBD combines a small profile (6-7 Fr), ability to expand in a small descending aorta and occlude relatively large PDAs (up to $5 \mathrm{~mm}$ ); it incorporates a folding plug over the button loop sutured at the center of the single wire occluder. After buttoning, the occluder remains at the aortic end of the ductus, the folding plug inside the ductal lumen and the counteroccluder in the pulmonary artery. The IBD was applied in 18 patients: ages $0.5-34$ yrs, weight $5-70 \mathrm{~kg}$, PDA-size 2-5 mm, IBD-size $15-20 \mathrm{~mm}$ and FO 18/18. All patients underwent the procedure without complications. The full occlusion rate was $100 \%$ within $24 \mathrm{hrs}$. In a $6 \mathrm{~kg}$ infant there was mild protrusion of the occluder-end in the aorta without gradient. In conclusion, the IBD has superior PDA occlusion rate than the regular device and is even applicable in young infants; because of its efficacy and safety, larger clinical trials are justified. 
III-06

Interventional atrioseptostomy using high frequency alternating current-in vitro evaluation

Grabitz RG, Eberhart A, Handt S, Seghaye MC, Franke A, von Bernuth $G$ Department of Pediatric Cardiology, Cardiology, and Pathology, Aachen Universty of Technology, Aachen and Dr. P. Osypka GmbH, Grenzach, Germany

The IAS (Rashkind procedure) today is limited to the newborn period. Later, with given indication, an open atrioseptectomy using cardiopulmonary bypass is needed with increased risks as well to the immediate procedure as to subsequent corrective surgery. We evaluated in vitro the use of HFC-Coagulation (HFC generator with temperature feed back control-HAT 200S, Dr. Osypka, Grenzach, Germany) via a symmetrical wire cage $(D=5 \mathrm{~mm}, \mathrm{~L}=20 \mathrm{~mm})$ in porcine atrial septum as a new IAS device. The cage, including a microthermistor, consists of six isolated monofile superelastic wires and is placed through a catheter into a punctured hole in the foramen ovale. Here the cage regains its volume shape. We measured the size of the defect depending on maximal temperature (up to $90^{\circ} \mathrm{C}$ ) and duration of current application (up to 60 $\mathrm{sec}$ ). Results (60 sec of HFC application) are summarized below.

$\begin{array}{ccr}\text { No. Preps } & \begin{array}{c}\text { Preset Temp } \\ \left({ }^{\circ} \mathrm{C}\right)\end{array} & \begin{array}{r}\text { Mean defec } \\ 15 \mathrm{~min} \text { af }\end{array} \\ 3 & \text { Control device }(2 \mathrm{~min}) & 4 \\ 3 & 30 & 3 \\ 5 & 50 & 8 \\ 7 & 70 & 18 \\ 2 & 90 & 17 \\ \text { Theoretical max size by shape of IAS device } & 25\end{array}$

Our new technical approach to IAS leads in vitro to reproducible results under circumstances (temperature, duration of current application) comparable with clinical interventional ablations. This rectifies further in vivo studies.

IV-01

Aerosolized prostacyclin for postoperative treatment in patients with pulmonary hypertension

Ublemann F, Schulze-Neick I, Stzller B, Berger F, Rossaint R, Hetzer R, Lange PE

German Heart Institute Berlin and the Department of Anesthesiology and Operative Intensive Care, Humboldt University, Berlin, Germany

Intravenous $\mathrm{PGI}_{2}$, a well-known vasodilator, can cause severe systemic hypotension when used for treatment ol PHT. The increasing clinical application of inhaled nitric oxide (iNO) has illustrated the physiology and benefits of selective pulmonary vasodilation. We examined the effects of aerosolized $\mathrm{PGI}_{2}$ (aePGI) in patients with postoperative $\mathrm{PHT}$ for selective right ventricular afterload reduction. We used aePGI in 7 patients with postoperatively persistent pulmonary hypertension of more than $33 \%$ of systemic pressure with ongoing NO therapy and steady hemodynamic and respiratory state; aePGI ${ }_{2}$ was applied using a

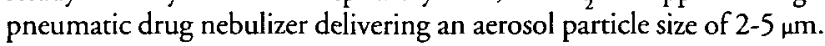
The aerosol was applied close to the Y-piece of the inspiratory limb in the ventilated patients. Results are summarized in the Table.

\begin{tabular}{|c|c|c|c|c|c|}
\hline & $\underset{(\mathrm{mm} \mathrm{Hg})}{\mathrm{pPA}}$ & $\begin{array}{c}\text { pPA } \\
(\% \text { pAo) }\end{array}$ & $\begin{array}{c}\mathrm{CVP} \\
(\mathrm{mm} \mathrm{Hg})\end{array}$ & $\underset{(\mathrm{mm} \mathrm{Hg})}{\mathrm{LA}}$ & $\begin{array}{c}\mathrm{Cl} \\
\left(\mathrm{L} / \mathrm{mn} \cdot \mathrm{m}^{2}\right)\end{array}$ \\
\hline INO & $35 \pm 3.5$ & $49 \pm 4.5$ & $8 \pm 11$ & $10 \pm 7$ & $3.42 \pm 1.04$ \\
\hline off & $54 \pm 4.6$ & $89 \pm 3.8$ & $9 \pm 17$ & $9 \pm 6$ & $2.92 \pm 0.63$ \\
\hline $\mathrm{aePGI}_{2}$ & $31 \pm 2.6$ & $42 \pm 3.3$ & $9 \pm 14$ & $10 \pm 8$ & $3.31 \pm 0.94$ \\
\hline
\end{tabular}

CI. cardiac index

In conclusion, it could be demonstrated that aePGI, exerts a relevant effect on pulmonary artery pressure, pulmonary vascular resistance, and indirectly on right ventricular function. In our limited experience, no acute side effects occurred. However, substantial further research is necessary to scientifically work up the benefits of either therapeutic strategy.
Notes 
IV-02

Nitric oxide-induced pulmonary vasodilator capacity to assess operability of children with severe pulmonary hypertension and elevated vascular resistance

Berner M, Beghetti M, Spahr I, Riemensberger P, Oberhaensli I, Friedli B Clinique Universitaire de Pédiatrie, Hôpital Cantonal, Geneva, Suitzerland

We used the nitric oxide (NO)-induced vasodilator capacity of the pulmonary vascular bed in addition to classical catheterization data to select candidates for operation in a group of children with longstanding pulmonary hypertension and elevated vascular resistance. Pulmonary vascular resistance $(\mathrm{Rp})$ and pulmonary-to-systemic vascular resistance ratio $(\mathrm{Rp} / \mathrm{Rs})$ were determined before and after a ten-minute inhalation of $\mathrm{NO}$ at $35 \mathrm{ppm}$, keeping $\mathrm{FiO}_{2}$ at 0.21 . A drop in both $\mathrm{Rp}$ and $\mathrm{Rp} / \mathrm{Rs}$ of more than $10 \%$ was regarded as a selective pulmonary vascular relaxation response. Nineteen children (8 with a VSD, 3 with DORV, 4 with an endocardial cushion defect, 1 with a PDA, 1 with single ventricle, 1 with primary PHT and 1 with idiopatic cardiomyopathy), aged 5 months to 16 years (median 41 months) were tested. All tolerated the test well and methemoglobinemia did not exceed $2 \%$ in any. Fourteen children were positive responders and decreased $\mathrm{Rp}$ from $9.2 \pm 1.1$ to $6.3 \pm 0.9$ units $\mathrm{m}^{2}, \mathrm{p}<0.05(-32 \%)$ and $\mathrm{Rp} / \mathrm{Rs}$ from $0.44 \pm 0.07$ to $0.28 \pm 0.05, p<0.05(-37 \%)$. Eleven of those whose Rp/Rs with NO reached 0.3 or less were considered candidates for repair of their congenital cardiac defect or for heart only transplantation. So far, 9 children have been operated on. All required NO postoperatively and one died from PHT. The 8 survivors were assessed 2 months after the operation, 2 by catheterization and 6 by echo. All had lowered pulmonary vascular resistance or systolic pulmonary artery pressure compared to preoperative values. Three children with a positive response and five nonresponders to $\mathrm{NO}$ who all had lowest $\mathrm{R} p / \mathrm{Rs}$ remaining above 0.3 were deemed inoperable. In conclusion, the type of response to NO varies among children with pulmonary hypertension and elevated vascular resistance and seems to add a valuable information to the assessment of operarive risk.

\section{IV-03}

Expression of myocardial protein isoforms in children with congenital heart defects

Kreuder J, Bode S, Borkhardt A, Hagel KJ, Paul T, Lampert F Children's Hospital, Justus-Liebig University, Giessen and Medical Schoob Hannover, Germany

Myocardial response to work overload includes the expression of protein isoforms. This isoform switch may result in different contractile properties of the myocardium. This study was to assess the relative distribution of myocardial isoforms of myosin heavy chain (MHC; alpha/beta type), myosin light chain 1 (MLC; atrial/ventricular type) and alphaactin (ACT; skeletal/cardiac type) in children with congenital heart defects undergoing cardiac surgery. The median $\mathrm{O}_{2}$ saturation was $91 \%$. Specific mRNAs were quantified by a competitive reverse transcriptionPCR assay using identical primers for both highly homologous isoforms, fragment separation by different restriction enzyme sites and fluorescencebased fragment length analysis. The expression of alpha-MHC (meant SEM:75.2+3.3vs7.3 $\pm 2.3 \%$; $<0.0001$ ), atrial $-M L C(78.5 \pm 6.2$ vs $40.5 \pm 8.5 \%$; $\mathrm{p}=0.013)$ and cardiac-ACT $(74.8 \pm 1.7$ vs $63.2+2.8 \% ; \mathrm{p}=0.0016)$ was higher in right atrial $(n=24)$ than in right ventricular $(n=16)$ myocardium. Increased right atrial pressure was associated with the expression of betaMHC $(r=0,69 ; p=0.0001)$ and skeletal-ACT $(r=0.58 ; p=0.0021)$ resembling the fetal pattern of contractile protein isoforms. Right ventricular tissue of patients with tetralogy of Fallot $(n=12)$ showed an unusual high proportion of up to $72 \%$ of alpha-MLC (fetal type) which has been shown to be associated with higher shortening velocity. A close relation ( $r=0.76 ; p=0.0002)$ existed between alpha-MLC and cardiacACT in right ventricular tissue. Reduced $\mathrm{O}_{2}$ saturation was associated with higher levels of skeletal-ACT (fetal type) in right atrial tissue $(r=0.45 ; \mathrm{p}=0.021)$. These findings demonstrate the molecular heterogeneity of myocardial contractile proteins in congenital heart defects. Analysis of myocardial protein expression may add to our understanding of myocardial function in these children.
IV-04

Mitral valve prolapse in children and adolescents

Zavrsnik J, Malcic I, Kancler K, Kokol P

House of Health and Faculty of Technical Sciences, Maribor, Slovenia,

Medical Faculty, University of Zagreb, Zagreb, Croatia

Consequences of mitral valve prolapse (MVP) are disturbed normal laminar flow, turbulence of the blood flow, injury of chordae tendinae, the possibility of thrombus composition, bacterial endocarditis and finally hemodynamic changes defined as mitral insufficiency and mitral regurgitation. Diagnosis is based on auscultatory findings confirmed by echocardiography. In Slovenia we started a research project to determine characteristics about the frequency and possible causes and consequences of MVP during childhood. Out of 38,000 newborns in the Maribor region, using the Monte Carlo method, we selected 900 children and adolescents, representing the whole population under eighteen years. We succeeded in examining 673 volunteers, 346 males and 327 females. All passed an examination of their state of health in the form of a carefully prepared protocol specially made for MVP syndrome. The protocol consisted of general data, mother' s health, father's health, pregnancy, delivery, postnatal period, injuries of chest or any other kind, chronic diseases, physical examination, subjective difficulties like headaches, chest-pain, palpitation, perspiring, dizziness, etc., auscultation, phonocardiography, ECG, and finally echocardiography. In that manner we gathered 107 parameters which can possibly indicate the presence of MVP. We detected 75 cases of MVP - 37 were male (11\%) and 38 were female $(12 \%)$. This finding is similar to recent studies in that there is no statistical significant difference between men and women according to the number of MVP cases. Using Chi-square and Student's t-test, we found that the following (alpha<0.05) significantly affect the appearance of the MVP syndrome: maternal diseases, cardiotonics during pregnancy, father's arrhythmia, Marfan's syndrome, dolichostenomelia, backbone malformations, dizziness, auscultation, weight, Rohr's index, chest circumference and heart rate.

IV-05

Issues of cardiovascular involvement in Kawasaki disease

Kato $H$

Department of Pediatrics, Kurume University, Kurume, Japan

The cardiovascular involvements in Kawasaki disease are most important clinical problems which may cause sudden death or ischemic heart disease in children. However, the long-term consequence of cardiovascular sequelae in KD remains uncertain at the present time. In 1973 we introduced coronary angiography $(C A G)$ as a routine cardiac examination of KD patients. From 1973 to 1994 we experienced 1,588 consecutive patients of acute KD, in whom $269(16.7 \%)$ had coronary aneurysms. These patients had been followed for more than 2 years with the longest 22 yrs (mean 6.1 yrs). Follow-up second CAG was performed in 211 , in whom 114 demonstrated regression of coronary aneurysms (54\%). Further follow-up CAG (3rd, 4th and 5th) were done in 75 cases. In the long-term parhological study, the regressed aneurysms revealed the marked intimal thickening mainly caused by proliferation of medial smooth muscle cells and well degenerated endothelium. Those findings were also demonstrated by intravascular ultrasound imaging in the follow-up patients. From this long-term follow-up study we review our data and discuss the following issues in the long-term problems of pediatric through adults in KD: (1) Cardiovascular spectrum in acute and subacute stage of illness; (2) fate of coronary aneurysms, regression of aneurysms and development to coronary artery disease; (3) $\mathrm{KD}$ vasculitis maybe an atherosclerotic risk factor; (4) the issues in the adult cardiology; (5) therapeutic recommendations. 


\section{IV-06}

Noninvasive detection of acute cardiac allograft rejection after pediatric heart transplantation by acoustic quantification

Netz H, Brangenberg R, Kozlik R, Schmitz C, Welz A, Reichart B Departments of Pediatric Cardiology and Cardiac Surgery, Großhadern Medical Center, University of Munich, Munich, Germany

The potential for noninvasive assessment of left ventricular dysfunction as an early marker for rejection has been considered. The HP Sonos 2500 ultrasound imaging system allows discrimination berween blood and tissue by acoustic quantification (AQ). In addition to echocardiographic measurements we used the AQ in 19 children and adolescents, aged 2 months to 19 years, who underwent heart (16) or heart and lung transplantation (3). Endomyocardial biopsies were performed per protocol or for suspected acute rejection. Special attention was directed to the time interval from the end of systole to the peak filling rate (TPFR, time to peak filling rate) as a parameter of the diastolic function. We compared 33 endomyocardial biopsies with the AQ results. In 23 cases there was no evidence of an acute rejection (grade 0 according to ISHT); 17 of these showed a left ventricular TPFR from $60-250 \mathrm{~ms}$ (apical fourchamber view) in the normal range of heal thy children. In 5 cases the left ventricular TPFR was shortened $(<60 \mathrm{~ms})$, associated with left or right ventricular hypertrophy, caused by arterial or pulmonary hypertension and stenosis of the aortic anastomosis. Ten endomyocardial biopsies revealed an acute allograft rejection - grade Ia (4), Ib (3), IIa (1), IIIa (2). In 9 cases TPFR ranged from 30-60 ms and was shortened, compared with the normal filling pattern. In one patient with left ventricular hypertrophy caused by stenosis of the aortic anastomosis, we found no further shortening of the TPFR $(60 \mathrm{~ms})$ during a mild acute rejection (grade Ib). In conclusion, after pediatric heart transplantation, acoustic quantification allows measurement of parameters of diastolic function, which appears to be highly sensitive in predicting acute cardiac allograft rejection. However, the reliability is reduced by ventricular hypertrophy, not caused by rejection.

\section{V-01}

Long-term results of cognitive and motor development in children after arterial switch operation for transposition of the great arteries Hövels-Gürich H, Seghaye MC, Däbritz $S$, Messmef BJ, von Bernuth $G$ Departments of Pediatric Cardiology and Cardiovascular Surgery, RWTH Aachen, Aachen, Germany

Between March 1986 and February 1992, 96 newborns with TGA underwent arterial switch operation in our institution in deep hypothermia and combined circulatoryarrest and low flow bypass. Of the surviving 88 patients, 52 unselected children were examined $61.1 \pm 19.7$ months after surgery. The neurological status was dinically evaluated. Standardized tests of cognitive function, gross motor function, fine motor skill and language were performed. Results were compared to age-matched normal children and related to pre-, peri- and postoperative parameters and events (Fishers Exact Test, independent Wilcoxon Test). The neurological starus was normal in $90.4 \%$, cognitive function in $90.4 \%$, gross motor function in $80.8 \%$, and fine motor skill in $82.7 \%$ of the 52 children. Cognitive and gross motor function (for which formalized tests are available) were not significandy different in the study group. Perinatal and preoperative course were not significantly related todevelopmental delay. Perioperativecomplicationshad asignificant influence on fine motor and gross motor function $(p<0.01)$. Late postoperative complications influenced significandy cogntive and gross motor function $(p=0.02)$. Degree of hypothermiaand circulatory arrest timedid notinfluence developmental outcome. Duration of bypass time significantly influenced cognitive $(p<0.005)$, fine motor $(p<0.01)$ and gross motor functions $(p<0.005)$. The study shows that developmental status of children after neonatal arterial switch operation is not significantly different from agematched normal children. Bypass duration, peri- and late postoperative complications were discriminative for later developmental dysfunctions. In our series, neonatal arterial switch operation in deep hypothermia with combined circulatory arrest and low flow bypass by itself did not result in developmental impairment in childhood.
Notes 
V-02

Long-term fate of the coronary arteries after the arterial switch operation in newborns - a selective coronary artery angiography study

Bonnet D, Bonhoeffer P. Piéchaud JF, Aggoun Y, Sidi D, Planché C, Kachaner J

Service de Cardiologie Pediatrique, Hôpital Necker, Enfants-Malades, Paris, France

Concern remains as to the long-term impact of the coronary artery $(\mathrm{CA})$ translocation after the arterial switch operation (ASO) for transposition of the great arteries. This study was conducted to determine the prevalence of obstructions of the translocated coronary arteries by using selective $\mathrm{CA}$ angiography. Sixty-four children (mean age 7.6 \pm 1.5 [SD] years) having survived an ASO underwent evaluation. They had been operated on by a single surgeon. They were followed-up by a single referring institution. Selective CA angiography was possible in 58 patients. Five patients exhibited occlusion or stenosis of a CA: 1 occlusion and 2 stenoses of the left coronary trunk, 2 occlusions of the circumflex artery. The prevalence of late CA complications is $7.8 \pm 6.6 \%$ (1.2 to $14.4 \%$, confidence limits at $95 \%$ ). The 3 patients with occlusion of one CA have had perioperative ischemic complications, with associated ECG evidence of ischemia and left ventricular dysfunction with mitral valve insufficiency. Both parients with stenosis of the left main $C A$ trunk did not have any suggestive anomaly before catheterization. In conclusion, the prevalence of the late CA complications after an ASO is low in our series. This supports our view that ASO remains the preferred treatment method for these patients. CA patency could have been predicted with reasonable certainty by using noninvasive methods. However, we believe that the late CA lesions should be screened by using selective CA angiography, as CA anomalies might be found even in patients who remain asymptomatic.

\section{$\mathrm{V}-03$}

Cardiorespiratory response to exercise after repair of tetralogy of Fallot-role of pulmonary regurgitation and right ventricular function Barat JL, Labbé L, Choussat A, Baudet E, Douard H, Broustet JP, Ducassou D

Hôpital Haut-Lévêque, Pessac, France

Fifty asymptomatic patients with repair of tetralogy of Fallot $(13 \pm 3$ years, 31 boys, 19 girls) were evaluated with treadmill exercise test with gas exchange measurement, rest two-dimensional and Doppler echocardiography and first-pass radionuclide ventriculography at rest and during exercise to determine the relation among exercise capacity, pulmonary regurgitation $(\mathrm{PR})$ and right ventricular performance. The mean age at repair was $4.3 \pm 2$ years; 24 patients had palliative operation prior to correction, and outflow tract reconstruction had been used in 26 patients. Exercise duration, peak oxygen consumption, anaerobic threshold, and maximal heart rate were significantly lower in patients in comparison with 50 normal children matched for sex, age and body surface area $(\mathrm{p}<0,01)$. PR was identified in $45 \mathrm{pts}$ and judged mild in 33 pts $(66 \%)$ and moderate in $12(24 \%)$. Peak oxygen consumption and anaerobic threshold were significantly lower in patients with moderate PR than in patients with mild or no regurgitation (respectively $29.5 \pm 4$ vs $38.5 \pm 5 \mathrm{ml} / \mathrm{kg} / \mathrm{min}, \mathrm{p}<0.01$, and $5.7 \pm 1.3$ vs $7.2 \pm 1.5 \mathrm{~min} ; \mathrm{p}<0.01$ ). Right ventricular ejection fraction (RVEF) was abnormal at rest in $20 \%$ of the patients. Right ventricle adaptation to exercise $(40 \%)$ was altered (RVEF increased less than $5 \%$ ) in $50 \%$ of the patients. Patients with abnormal rest RVEF had significantly lower peak oxygen consumption $(p<0.001)$. Age at repair, prior palliative surgery, type of repair did not correlate with echocardiographic variables, right ventricular performance or cardiorespiratory response to exercise. We conclude that PR and right ventricular function limit exercise ability after repair of tetralogy of Fallot.
V-04

Pulmonary atresia with intact septum-growth of the right ventricle and tricuspid valve following primary procedure

Daubeney PEF, Slavik Z, Keeton BR, Anderson RH, Webber SA $U K$ and Eire Collaborative Study of Pulmonary Atresia With Intact Ventricular Septum (PAIVS), Wessex Cardiothoracic Centre, Southampton, United Kingdom

The initial surgical strategy for PAIVS is critical not only for survival but also for the growth potential of the right ventricle (RV) and tricuspid valve (TV). We examined the growth of the TV and RV following primary procedure. Serial echoes were reviewed in 61 children both preand post-primary procedure (mean 17 months post-procedure, range 245). The TV annulus and RV inlet length were measured in the fourchamber view. Z-scores were derived from published nomograms based on surface area. The initial TVZ-score was significantly smaller in those undergoing shunts than in those undergoing various RV outflow tract procedures (RVOT) with or without concomitant shunt $(-2.2 \pm 0.3$, $-0.8 \pm 0.2$ respectively, $p=0.0002$ [data shown as mean \pm SEM]). The change in TV Z-score post-procedure was similar (shunt $-0.7 \pm 0.4$, RVOT $-0.8 \pm 0.2, p=N S$ ). Similarly, the RV inlet $Z$-score was smaller in the shunt group than the RVOT group $(-1.6 \pm 0.1,-1.2 \pm 0.1$ respectively, $p=0.0009$ ). Again, the change in the $Z$-scorewassimilar (shunt $-0.06 \pm 0.1$, RVOT $-0.3 \pm 0.1, p=N S$ ). Of those patients undergoing RVOT alone, neither type of procedure (catheter versus surgery), nor requirement for a shunt within $6 \mathrm{wks}$ of procedure affected the change in TV or RV inlet Z-score. In patients with primary RVOT, change in TV and RV inlet Z-score was weakly correlated with $\mathrm{TV}$ and $\mathrm{RVZ} Z$-scores pre-procedure. Larger increases in TV and RV inlet size post-procedure were associated with smaller initial TV and RV inlet sizes $(r=0.47, p=0.01$ and $r=0.44$, $\mathrm{p}=0.01$ ). There was no such correlation in patients shunted alone. Following establishment of RV to PA continuity, growth of the TV and $\mathrm{RV}$ frequently does not exceed somaticgrowth but is more likely in those ventricles with small initial TVs and RVs.

V-05

Determination of intrapulmonary and intracardiac right-to-left shunts following total cavopulmonary connection

Hofbeck $M$, Buheitel $G$, Singer H, van dern EmdeJ Departments of Pediatric Cardiology and Cardiac Surgery, University Erlangen, Nürnberg, Germany

The aim of this study was to assess the amount of intrapulmonary and intracardiac RL-shunts in the medium-term follow-up after TCPC. We examined 14 pts who underwent elective cardiac catheterization with a mean interval of 43 months (range 26-61) after the modified Fontan procedure. All pts underwent complete right and left heart catherization. The shunts were calculated based on Fick's principle. Angiographies were performed in all patients to show the anatomy of the TCPC and the pulmonary arteries. Systemic venous collaterals were injected selectively. The total RL-shunt ranged from $11-24 \%$ (mean $18.4 \%$ ) with an intrapulmonary portion of 6-18\% (mean 9.6\%) and an intracardiac portion of $4-17 \%$ (mean $8.8 \%$ ). None of our pts had obstruction of the TCPC or intrapulmonary arteriovenous fistulae demonstrated by angiography. Eight pts had angiographic evidence of small (6 pts) or significant (2 pts) leaks of the intraatrial tunnel. In 5 pts we found systemic venous collaterals entering pulmonary veins, the coronary sinus or the left atrium. Three of these pts had an intracardiac RL-shunt $>10 \%$ without evidence of a significant tunnel leak. There was no significant difference in the central venous pressure between those pts with systemic venous collaterals (mean $10.8 \mathrm{~mm} \mathrm{Hg}$ ) and the rest of the cohort (mean $11.2 \mathrm{~mm} \mathrm{Hg}$ ). All 14 pts in our medium-term follow-up after TCPC had an increased total RL-shunt. The intrapulmonary portion of the RL-shunt was $>10 \%$ in $7 / 14$ pts despite the absence of pulmonary arteriovenous fistulae. Possible sources of an intracardiac RL-shunt are the coronary sinus, leaks of the intraatrial tunnel and systemic venous collaterals. The latter may develop in the absence of significant elevation of central venous pressures and may necessitate interventional occlusion. 
V-06

Familial non-syndromic conotruncal defects are not associated with a 22q11 microdeletion

Debrus $S$, Berger $G$, de Meeus A, Sauer U, Guillaumont $S$, Voisin $M$, Bozio A, Bouvagnet $P$

Service de Pédiatrie II, Hôpital St Charles, Montpellier, Service de Cardiologie C, Hôpital Cardiologique, Lyon, France and Kinderklinik, Deutsches Herzzentrum, München, Germany

Molecular studies have shown microdeletions in region q 11 of chromosome 22 in nearly all patients with DiGeorge, VeloCardioFacial and ConoTruncal Anomaly Face Syndromes (DGS, VCFS and CTAFS, respectively) and in a high percentage of non-syndromic familial cases of conotruncal defects (CTD). CTD account for roughly a fourth to a third of all non-syndromic Congenital heart defects (CHD), thus 22q 11 could harbor a major genetic factor of CHD. We searched for a $22 q 11$ microdeletion in familial cases of non-syndromic CTD. Thirty-six cases of various isolated CTD, that is without history of hypocalcemia, immune deficiency, absent thymus, and dysmorphic appearance were selected. With 48F8, a cosmid probe localized in the smallest deleted region of the DiGeorge critical region (DGCR), we found no deletions by FISH in these 36 affected individuals of 16 families with recurrent CTD. Moreover, D22S264, a microsatellite localized at the distal part of the largest deleted region, was used to genotype the patients. Thirtytwo of 37 patients were heterozygous and hence not deleted at this locus, whereas 5 were uninformative. In conclusion, there is no large deletions in familial cases of various CTD whether these defects are identical or not within a family. This result does not rule out other minor anomalies in this chromosomal region.

\section{V-07}

Systemic venous collaterals after the bidirectional cavopulmonary anastomosis prevalence and risk factors

Magee AG, McCrindle BW, Benson LN, Williams WG, Freedom RM Hospital for Sick Children, Toronto, Canada

To determine the prevalence and risk factors associated with the development of systemic venous collaterals (SC) after a bidirectional cavopulmonary anastomosis (BCPA), catheterization data were reviewed on 58 patients who underwent BCPA between 1982 and 1994. The median age at BCPA was 17 months (range 4-172) and the postoperative study performed after a median of 13 months (range <1-44). SC were seen in 11 (19\%) patients and included hemiazygous, azygous, pericardial, mediastinal, superior intercostal, paravertebral and diaphragmatic veins. Risk factors assessed for SC included age at surgery, time postoperative, dominant ventricular morphology, type of superior vena cava (SVC) connection, presence of pulsatile flow, superior vena caval, mean pulmonary artery (PAP) and ventricular end-diastolic pressures (EDP), arterial oxygen saturation, and pulmonary artery (Nakata) index. SC were significantly associated with bilateral SVC (50 vs $11 \%$ for RSVC; $p=0.006$ ), lower mean postoperative aortic saturation ( $76 \pm 19$ vs $87 \pm 6 \% ; p=0.001)$, a higher mean postoperative PAP ( $17 \pm 5$ vs $11 \pm 4 \mathrm{~mm} \mathrm{Hg} ; \mathrm{p}=0.002$ ), higher postoperative EDP ( $11 \pm 6$ vs $8 \pm 3 \mathrm{~mm} \mathrm{Hg} ; \mathrm{p}=0.04$ ), lower median post-operative Nakata index (205 vs $326, \mathrm{p}=0.08)$ and shorter median postoperative interval (5 vs 14 months; $\mathrm{p}=0.01$ ). Independent factors from multiple logistic regression included bilateral SVC (odds ratio $7.83,95 \% \mathrm{Cl} 1.56$ to $39.3 ; \mathrm{p}=0.01$ ) and high postoperative PAP (OR per $5 \mathrm{~mm} \mathrm{Hg} 2.66,95 \%$ CI 1.20 to $5.88 ; \mathrm{p}=0.02)$. SCare not uncommon after $\mathrm{BCPA}$, reduce postoperative systemic arterial oxygen saturations and are associated with bilateral cavae and higher postoperative PAP.

\section{Notes}


VI-01

Percutaneous transfemoral balloon angioplasty for aortic coarctation in adults

Digiovanni JV, Gupta J, Watson RDS, Lip GYH, Osman K, Slim IF, Mohan M, Singh SP

Department of Cardiology, City Hospital NHS Trust, Birmingham, UK

Over a five-year period we considered percutaneous balloon angioplasty (PBA) in 27 adults (17 male, 10 female; mean age 31.8, range 15-58) with aortic coarctation. Twenty-four patients were newly diagnosed and 3 had recurrences after surgery (Dacron graft, Dacron patch, resection anastomosis). Dilatation was successfully carried out in 23 patients ( 15 male, 8 female). The procedure was not attempted in four patients: one due to a double bend at the coarctation site, the other due to calcification at the narrowed segment with a patent ductus, a third due to complete obstruction, and a fourth due to the gradient being less than $10 \mathrm{~mm} \mathrm{Hg}$ on cardiac catheterisation. The first 3 were successfully managed surgically. The mean predilatation coarctation gradient was $46.2 \pm 13.6$ $\mathrm{mm} \mathrm{Hg}$ with a fall post-dilatation to a mean of $9.9 \pm 10.2 \mathrm{~mm} \mathrm{Hg}$ (mean reduction $36.2 \pm 12.2 \mathrm{~mm} \mathrm{Hg} ; \mathrm{p}<0.0001$ ) Immediate reduction in gradient to $<20 \mathrm{~mm} \mathrm{Hg}$ was achieved in 21 patients; 2 patients needed repeat procedures. There were no significant complications following the procedure. After a mean follow-up of 32.5 months (range 17 to 49 months), Doppler gradients of the descending aorta systemic blood pressure (brachial) and ankle occlusion pressures were determined. There was a reduction in mean systemic BP (mean reduction of $-28.9 \pm 12.8 \mathrm{~mm} \mathrm{Hg} ; \mathrm{p}<0.0001$ ) and mean DBP (mean reduction $-12.4 \pm 6.9 ; \mathrm{p}<0.0001)$. There was no significant difference between brachial SBP and ankle occlusion pressures $(\mathrm{p}=0.11)$. Antihypertensive therapy was discontinued in 11 patients and reduced in 12 patients. Our experience suggests that PBA is a feasible and safe procedure in adults in coarctation of the aorta. The procedure should be increasingly considered in adults with this condition.

VI-02

Evaluation of pulmonary hypertension with inhaled nitric oxide, intravenous and aerosolized prostacyclin

Schulze-Neick I, Nürnberg J, Opitz C, Berger F, Dähnert I,

Ublemann F, Kleber FX, Rossaint R, Hetzer R, Lange PE

German Heart Institute Berlin, Catheterization Laboratory, Department of Internal Medicine and Department of Anesthesiology and Operative Intensive Care, Humboldt University, Berlin, Germany

Evaluation of pulmonary vasoreactivity in pts with primary PHT (pPHT) or PHT caused by congenital heart disease (CHD) is required to determine further therapy including conservative medical therapy, heart operation, or transplantation. We have developed a routine steplike protocol for evaluation of pulmonary vasoreactivity which includes intravenous (non-selective) as well as inhaled (selective) vasodilators. Fifty-six pts were catheterized and tested using inhaled oxygen, intravenous prostacyclin and at least one of the inhaled vasodilators ( $\mathrm{aePGI}_{2} /$ iNO); 21 pts received both. After measurements at room air, oxygen, additionally iNO (50 ppm for 5-10 min), then aePGI $(10 \mathrm{~min})$, and finally ivPGI ${ }_{2}(10-15 \mathrm{ng} / \mathrm{kg} / \mathrm{min})$ were given. $\mathrm{NO}$ and $\mathrm{NO}_{2}$-concentration was monitored, and aePGI ${ }_{2}$ was applied by a jet nebulizer producing particles of $2-5 \mu \mathrm{m}$. Results are listed in the Table.

\begin{tabular}{|c|c|c|c|c|c|c|}
\hline & $\begin{array}{c}\text { SacAo } \\
(\%)\end{array}$ & $\begin{array}{c}\mathrm{pPA} \\
(\% \mathrm{PAO})\end{array}$ & $R_{p} \cdot R_{s}$ & $\begin{array}{c}\text { \% Rp:Rs } \\
\text { change }\end{array}$ & $\underset{\left(L / m ı n \cdot m^{2}\right)}{C I}$ & $\begin{array}{l}\text { PVR } \\
\text { (WU) }\end{array}$ \\
\hline Air & $918 \pm 42$ & $78 \pm 22$ & $0.75 \pm 0.37$ & & $2.73 \pm 0.95$ & $199 \pm 16$ \\
\hline $\mathrm{O}_{2}$ alone & $986 \pm 26$ & $78 \pm 24$ & $0.55 \pm 0.27$ & $26 \pm 21$ & $334 \pm 0.47$ & $161 \pm 4.1$ \\
\hline${ }_{1} \mathrm{NO}_{2} \mathrm{O}_{2}$ & $981 \pm 36$ & $74 \pm 24$ & $0.52 \pm 0.27$ & $30 \pm 22$ & $424 \pm 112$ & $122 \pm 1.9$ \\
\hline aePGI $/ \mathrm{O}_{2}$ & $991 \pm 2.3$ & $74 \pm 24$ & $0.46 \pm 0.27$ & $37 \pm 24$ & $411 \pm 111$ & $12.1 \pm 3.9$ \\
\hline IvPGI $/ \mathrm{O}_{2}$ & $973 \pm 4.5$ & $80 \pm 22$ & $0.59 \pm 0.34$ & $17 \pm 32$ & $423 \pm 1.11$ & $13.2 \pm 3.8$ \\
\hline
\end{tabular}

$\mathrm{AePGI}_{2}$ exerts a relevant effect on pulmonary artery pressure, pulmonary vascular resistance, and cardiacoutput. Despite marked inter-individual differences of maximum effect, aePGI, revealed slightly more pulmonary vasoreactivity than iNO. Further studies are necessary to safely establish the promising therapeutic role of aePGI $\mathrm{PI}_{2}$ for indications similar to iNO, but with less complicated mode of application.
VI-03

Depolarization-repolarization inhomogeneity leads to ventricular arrhythmia after repair of tetralogy of Fallot

Gatzoulis $M A$, Till JA, Redington AN

Royal Brompton Hospital, London, United Kingdom

We have previously shown that QRS prolongation $(>180 \mathrm{~ms})$ is a very sensitive and relatively specific risk marker for malignant ventricular tachycardia (VT) after repair of tetralogy of Fallot (rTOF). We have now examined the dispersion (d) of QT and its components QRS and JT in an attempt to identify the relative contributions of depolarization and repolarization abnormalities to the pathogenesis of VT in these pts. QRS width and QT/QRS/JT $d$ were measured manually from standard ECGs in 10 syncopal rTOF pts (group 1) [21.4 4.6 years after repair] with QRS $>180 \mathrm{~ms}$ and with documented VT, and compared with 9 rTOF pts with QRS $>180 \mathrm{~ms}$ and no VT (group 2), 40 rTOF pts with QRS $<180 \mathrm{~ms}$ and no clinical arrhythmias (group 3), and 40 non-rTOF controls. Mean QT d $(62.7 \pm 36.5 \mathrm{~ms})$ in the rTOF pts was greater than in controls $(34.4 \pm 10 \mathrm{~ms}, \mathrm{p}<0.001)$. The Table shows *significant $\mathrm{p}$ values $<0.001-0.01$, compared with group 1 :

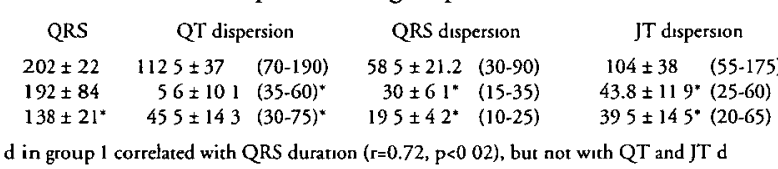

QT/QRS/JT d were greater in rTOF pts with VT. JT and QT $d$ in this group were independent of QRS duration and $\mathrm{d}$, suggesting that both depolarization and repolarization abnormalities may participate independently in the pathogenesis of VT after rTOF. Furthermore, a QRS duration $\geq 180 \mathrm{mscombined}$ with a $\mathrm{QT}>60 \mathrm{~ms}$ or $\mathrm{QRS}>35 \mathrm{~ms}$ or $\mathrm{JT}>60$ ms was $98.3 \%$ sensitive and $100 \%$ specific for identification of pts with VT from our large rTOF data set.

VI-04

Safety of amiodarone therapy in infancy

Villain E, Bonnet D, Aggoun Y, Iserin L, Kachaner J

Hôpital Necker, Enfants Malades, Paris, France

Due to possible adverse side effects, the use of amiodarone in children has been restricted to critical or intractable arrhythmias. To assess its efficacy and toxicity in the young age, we reviewed the records of 60 infants who received amiodarone. Fifty-three newborns and 7 infants less than 6 months were admitted for atrial arrhythmias $(8 \mathrm{pts})$, supraventricular re-entry tachycardia ( $45 \mathrm{pts}$ ), junctional or ventricular incessant tachycardia $(7 \mathrm{pts})$. All had normal heart structures. Oral amiodarone was started in hospital at a loading dose of $500 \mathrm{mg} / \mathrm{m}^{2}$ during 10 days, followed by a maintenance dose of $200-250 \mathrm{mg} / \mathrm{m}^{2}$. In half the cases, amiodarone was the first choice drug and it was given after failure of digoxin in the other half. Duration of treatment varied from 6 to 60 (mean 20, median 12) months. Amiodarone was effective in all children and no proarrhythmia was recorded; one pt had bradycardia $<80 / \mathrm{min}$ and amiodarone was stopped. Seven pts developed abnormal thyroid function tests: 5 had a mild and transient elevation of TSH $<10$ $\mathrm{IU} / \mathrm{ml}$ and 2 had biological hypothyroidism with normal T 3 and T 4 and high TSH; one required a temporary treatment with L-thyroxin but amiodarone was not stopped. In 2 infants, a negative deviation of the curves of growth and height velocity was observed and returned to normal after the treatment was stopped; both had normal thyroid function. There was no skin sensitivity and chest $X$-ray remained normal in all cases. In conclusion, amiodarone was found to be very effective and safe in infants. Thus, it may be proposed not only for treatment of refractory arrhythmias but also as a first choice therapy to prevent supraventricular tachycardia in the first year of life. 


\section{VI-05}

Pacemaker survival in permanent cardiac pacing after surgery for congenital heart defects

Janousek J, Hucin B, Tax P, Tlaskal T, Reich O, Vojtovic P, Kostelka M Kardiocentrum, University Hospital Motol, Prague, Czech Republic

To evaluate long-term results of permanent cardiac pacing in children after surgery for congenital heart defects, 41 consecutive pts with pacemakers implanted from 1978 to 1994 for sinus node dysfunction (9), atrioventricular block (30) or both (2) at median age of 6.2 yrs $(0.2$ mo-17.3 yrs) were followed for a mean of 48.4 (1.0-159.7) months. A total of 51 pulse generators (AAI 3, WI 34, WIR 2, DDD 9 and DDDR 3), 14 atrial and 50 ventricular (endocardial 34, epicardial 30) pacing leads were implanted during 58 procedures. Actuarial survival curves were used to evaluate risk factors for surgical reinterventions. Seventeen postimplant interventions (pacemaker/lead removal, reimplantation or revision) had to be performed in 13 pts (31.7\%) for following (combined) reasons: high threshold (6), battery depletion (5), pocket erosion (3), infection (2) and pacemaker failure, pacemaker syndrome and lead extension into pulmonary artery each once. Intervention-free survival was $80.1 \%$ at 1 yr and $63.2 \%$ at 5 yrs after implantation. Subcutaneous (versus submuscular) pocket tended to be associated with more interventions due to pocket erosion or infection. Epicardial leads had more early failures due to exit block than endocardial, but long-term performance and need for generator replacement due to battery depletion were similar in both groups. All atrial leads were functional at a mean of 22.0 (6.2-66.6) mo after implantation. All DDD paced pts remained in DDD mode during a mean follow-up of 17.9 (6.2-66.6) months. In conclusion, postimplant surgical interventions were necessary in onethird of pts. Submuscular pocket seemed to be better than subcutaneous. Use of epicardial or atrial leads and dual chamber pacing were not associated with more postimplant interventions on long-term followup. Physiological pacing could be achieved without risk of more postimplant complications.

\section{VI-06}

The cloning of a new regulatory gene with a with a potential role in the control of myocardial differentiation and growth

Huggon IC, Towner P, Moscoso G, Farzaneh F, Tynan M Guy's Hospital and King's College Hospital Medical School, London, United Kingdom

The aim of this research was to isolate and characterize novel genes controlling differentiation and growth in myocardium. Elucidation of such underlying molecular mechanisms may prove vital to future satisfactory treatment for obstructive congenital heart lesions in which failure of adequate and normal growth of a ventricle is a feature. Degenerate oligonucleotide primers for use in the polymerase chain reaction (PCR) were designed to stretches of amino acid sequence conserved within a family of gene regulatory molecules in an attempt to isolate genes for different but related factors expressed in developing human heart. From a 340 base-pair gene fragment isolated in this way, the full length transcript of a novel gene was cloned using cDNA library screening and PCR based methods. Northern blot analysis and in-situ hybridization studies demonstrated a tissue restricted partern of expression confined to heart and derivatives of the developing gut. Sequence analysis confirms homology to members of the GATA-gene family and allows designation of the gene as human GATA-6. Other members of the GATA-family are known to act in other tissue types by regulating the expression of the complete set of genes responsible for the structural and functional proteins required for initiating and maintaining the differentiated characteristics of cells. Recent work by others showing that the closest relative, GATA-4 is able to influence the expression of some cardiac specific genes in mouse supports the hypothesis that GATA- 6 also functions in myocardial differentiation. Human GATA- 6 gene is strongly expressed in developing heart and has the characteristics of a regulator of differentiation and growth.
Notes 


\section{VI-07}

Incidence and clinical consequences of graft coronary arterial disease in children

Le Bidois J, Piéchaud JF, Sidi D, Tamisier D, Voubé P, Kachaner J Hôpital Necker-Enfants Malades and Hôpital Laënnec, Paris, France

We studied coronary angiograms (angio) and cardiac events in 43 patients with a follow-up $>1$ year after 46 heart transplantations (HT). In 13 more recent cases, a baseline angio was obtained within 3 months of HT. In our early experience, patients had their first angio after up to 4 years after HT. Angio showed obvious lesions of GCAD in 8 patients. GCAD was first detected at 1 year in 3 patients, 3 years in 2 patients, 4 years, 5 years, and 8 years in the 3 remaining patients. Four patients were retransplanted 17-81 months after their first HT; 2/4 died suddenly at 20 and 26 months after retransplantation. One patient died 45 months after HT. One patient is listed for retransplantation. One patient has discrete stenoses of the left anterior descending coronary artery and is scheduled for angioplasty. One patient has mild stable lesions. During the study period, 1 patient with no angiographic GCAD died suddenly 21 months after HT and 1 patient (not included in the study population) died at 11 months of myocardial infarction. In conclusion, moderate to severe GCAD is frequent and prognosis is poor. Coronary angiograms should be repeated annually in order to propose retransplantation or coronary angioplasty to selected patients.

VII-01

Cerebrovascular resistance in infants before and after cardiac surgery by means of cardiopulmonary bypass—relation to occurrence of cerebral injury

Abdul-Khaliq H, Gamillscheg A, Ublemann F, Langer PE Department of Pediatric Cardiology, Deutsches Herzzentrum Berlin, Germany

Early prediction of cerebral injury associated with repair of congenital heart defects in neonates and infants remains of great importance. Measurement of cerebral blood flow velocity (CBFV) in the major cerebral arteries provides noninvasive information on cerebral blood flow and resistance. To determine the relationship between alterations of cerebrovascular hemodynamic and subsequent occurrence of visible cerebral injury, in 58 pts below the age of 8 months (d-TGA, $\mathrm{n}=27$; complete atrioventricular septal defects [CAVSD], $\mathrm{n}=16$; VSD, $\mathrm{n}=15$ ), serial transfontanel measurements of CBFV in the anterior cerebral artery (ACA) and internal carotid artery (ICA) were performed using colour Doppler 24 hours before surgery and then at $30 \mathrm{~min}$, $1,2,4,8$, and 24 hours, and then daily for 6 days. The flow velocity internal of the area under the velocity curve (FV) and the resistance index $(\mathrm{RI}=\mathrm{Vmax}-$ $\mathrm{Ved} / \mathrm{V}_{\max }$ ), were used as actual qualitative parameters of cerebral blood flow and resistance. CBFV decreased significantly immediately after surgery $(p<0.01)$ and returned to the preoperative values between $48-120$ hours postoperatively. The increased RI persisted until the 5 th postoperative day in infants with CAVSD and d-TGA without IVH, and normalized during the 24 hours in infants with VSDs. Moderate IVH grade I-III was diagnosed in 9 newborn infants with d-TGA after primary switch op within the first 24 hours postoperatively. The RI values in thoseptswhosubsequentlydeveloped IVH were significantly lower than those pts who did not have IVH $(p<0.01)$. In conclusion, the significant decrease of FVI and increase in RI during the early postoperative period may indicate alteration in cerebral hemodynamic following cardiac surgery by means of $\mathrm{CPB}$; lower RI values pior to the onser of IVH in neonates with $\mathrm{d}-\mathrm{TGA}$ may indicate vasodilation and possible impairment of autoregulation; and serial determinations of CBFV in hemodynamically unstable infants could help to predict those at increased risk.
VII-02

Left ventricular mechanics after the Ross procedure for aortic valve incompetence

Shango P, Sluysmans T, Rubay J, Lintermans J, Vliers $A$

UCL St Luc, Brussels, Belgzum

The aim of this study was to analyse the benefits on LV function of the Ross procedure in congenital patients (pts) with aortic valve insufficiency. From April 1993 to March 1995, aortic valve replacement with autograft (Ross procedure) was performed for congenital anomalies resulting in aortic incompetence in 18 pts (median age 10 years, range 5 months to 22 years). Echocardiography was obtained preoperatively and serially up to one year after surgery to study LV function. One pt died in the early postoperative period (5\%). All survivors remained in NYHA class I and were free of complications and on no medication. No gradient nor any significant aortic incompetence could be demonstrated. End-diastolic LV dimensions (EDD) diminished drastically from $2 \pm 2.6$ above normal to $-0.63 \pm 2$ at one week postoperatively (D7). LV mass remained abnormal at D7 (from $4 \pm 3$ to $3.6 \pm 3$ ) and diminished more progressively to reach normal values $(0 \pm 1.5)$ at 1 month. This resulted in a significant decrease of end-systolic wall stress $(-4 \pm 2)$ and in a hyperdynamic function in the immediate postoperative days except in 2 pts. LV fractional shortening (FS) remained low $(<25 \%)$ at long-term follow-up in those 2 pts. Before surgery, those 2 pts had normal FS, a more dilated LV (EDD $5.3 \pm 1.95$ vs 1.6 \pm 2 ), decreased LV wall thickness (EDW) with a decreased EDW/EDD ratio (0.14 \pm 0.06 vs $0.2 \pm 0.06)$, a more spherical LV, a decreased velocity of shortening (VCFc 0.83 vs $1.19 \mathrm{cycle} / \mathrm{sec}$ ). They were the only 2 pts with decreased contractility as indicated by abnormal (<-2SD) LV end-systolic circumferential stress (ESSc) -VCFc relation. In conclusion, the Ross procedure suppresses completely the abnormal LV myocardial mechanics associated with volume-load in aortic incompetence in most pts. Surgery should be performed before deterioration of $\mathrm{LV}$ contractility, indicated by an abnormal ESSc-VCFc relation.

VII-03

Restrictive right ventricular physiology after repair of tetralogy of Fallot Norgard G, Gatzoulis MA, Moraes F, Lincoln C, Shore DF, Shinebourne EA, Redington $A N$ Royal Brompton Hospital, London, United Kingdom

We have recently reported that a restrictive right ventricle (RV) late after tetralogy of Fallot reduces pulmonary regurgitation, QRS duration and late arrhythmias. Conversely, QRS prolongation is a sensitive marker of RV dilatation and late arrhythmias. The aim of this study was to assess determinants of restrictive RV physiology in TOF patients repaired in our hospital during the last 10 years. We have studied 88 patients 1 month to nine years (median 1.6 yrs) after repair with Doppler echocardiography. Age at surgical repair was from 2 months to 43 years (1.9 yrs). Restrictive RV physiology defined as antegrade diastolic pulmonary artery flow with atrial systole was found in 28 patients $(32 \%)$, and was unrelated to age at repair, previous shunt $(n=19)$, crossclamp or bypass time. However, 16 of 39 patients (41\%) with a transannular patch had a restrictive $R V$ as compared to 2 of $25(8 \%)$ with outflow tract repair only $(\mathrm{p}<0.05)$. The $Q R S$ duration $(\mathrm{ms})$ in patients with restrictive and nonrestrictive RV was related to type of repair.

$\begin{array}{lccc} & \text { Transannular patch } & \text { Monocusp } & \text { Outflow patch } \\ \text { Restrictive } & 119 \pm 18.6 & 116.3 \pm 18.9 & 120.0 \pm 6.9 \\ \text { Nonrestrictive } & 133.3 \pm 12.2 & 121.4 \pm 32.0 & 123.1 \pm 18.4 \\ \text { p value } & <0.02 & \text { NS } & \text { NS }\end{array}$

In conclusion, restrictive RV physiology is more common after TOF repair with a transannular patch. Nonetheless, nonrestrictive physiology in the presence of a transannular patch significantly increases QRS duration with potential deleterious long-term features. 
VII-04

Is routine preoperative transesophageal echocardiography cost-effective? Söderberg B, Mellander M, Grönstedt A, Andreasson S, Suidow $G$ Departments of Pediatric Cardiology, Thoracic Surgery and Pediatric Anesthesiology, Östra Sjukhuset, Göteborg, Sweden

Since the start in September 1994 until July 1995 we performed 225 TEE. Forty were done in association with catheter interventions (ASD closure), myocardial biopsies or in the intensive care unit. The rest (184) were peroperative TEE performed immediately before and/or after bypass. We report the results of those TEE that were done immediately after bypass. Out of 176 open heart procedures, postoperative TEE was done in 74 (group A) and not done in 102 (group B). In group A (74), the TEE result convinced the surgeon to go back on bypass for a revision in one case (residual VSDs). In this group there were 2 reoperations and 4 deaths. The indications for reoperation (1 late postoperative thrombosis and 1 VSD-patch detachment) were not present at the time of TEE. In 3 of the 4 deaths, TEE showed depressed myocardial contractility but no residual anatomical lesion. In one of these patients, postmortem examination revealed a surgical obstruction of the left coronary artery, but in the other 3 no anatomical explanation was found. The fourth patient died late after surgery from septicemia. In group B (102), there were 9 reoperations. In 5 of these, TEE might have led to a surgical revision at the primary operation, whereas in the other 4 the indication for reoperation was not present at the first postoperative transthoracic echocardiogram. In pts not reoperated there were 4 deaths. In only one of these pts, postmortem revealed an anatomical problem which might have been detected on TEE. In conclusion, the maximal possible benefit of routine peroperative TEE in this series of 176 open-heart procedures would be to prevent 6 reoperations and one death. We propose that this could have been accomplished with a protocol of perioperative TEE on selected indications (AV valve and VSD surgery). This would reduce the number of postoperative TEE by $50 \%$.

\section{VII-05}

Three-dimensional echocardiography provides new information on morphology of complete atrioventricular septal defect

Vogel M, Ho SY, Anderson RH

Department of Paediatrics, German Heart Center, Berlin, Germany and National Heart and Lung Institute, London, United Kingdom

We evaluated whether three-dimensional (3D) echocardiography can provide additional information over 2D-echo on anatomy of atrioventricular septal defect (AVSD) in 32 patients aged 0.2-13 (median 0.8) years and 3 autopsy specimens. The tomographic ultrasound probe acquires parallel images of the heart steered by a stepper motor, which moves the probe in $0.5 \mathrm{~mm}$ steps with ECG-and respiration-gating; 80 100 parallel slices of the heart were thus obtained, which form the 3Ddataset. The AVSD could be displayed in new views: (a) as seen through a right atriotomy simulating a surgical view; (b) the AV valves could be displayed as if seen en face; and (c) the ventricular septal defect component of the AVSD was displayed as if viewed from either ventricle. Additional information was thus obtained on $A V$-valve morphology, especially on the distance between anterior (superior) and posterior (inferior) common bridging leaflet, which determines the size of the "cleft;" and, more importantly, on the size of the left mural leaflet and the component of the posterior bridging leaflet committed to the left ventricle, which, if small, leads to important left AV-valve regurgitation and is very difficult to repair. In 2 patients with small $L V$, the en face view of the AV valves demonstrated the small component of the common $A V$ valve committed to the $L V$ cavity. $3 D$-echo proved useful in delineating the mechanism of partial dynamic or complete closure of ventricular component of AVSD by AV valve leaflets and their respective tension apparatus. We conclude that $3 \mathrm{D}$-echo offers diagnostic information superior to 2D-echo on AVSD anatomy and can thus aid in better planning surgical therapy.

\section{Notes}




\section{VII-06}

Histomorphometric study of pulmonary vessels in patients with univentricular heart and low pulmonary blood flow-influence on surgical management

Lévy $M$, Vernant $F$, Carnot $F$, Danel $C$, Voubé P, Leca $F$ Laënnec Hospital, Paris, France

In children with univentricular heart and low pulmonary blood flow, surgical indications are usually based on hemodynamic data. Structural changes in the pulmonary vascular bed, however, may influence the outcome. The present study was undertaken (1) to assess the relationship between hemodynamic and histologic findings and (2) to evaluate the interest of histomorphometric study for the surgical decision making. An open lung biopsy was obtained in 28 patients aged 6 months to 16 years (mean 7.2 \pm 4.4 years) with univentricular heart (17) or tricuspid atresia (11) who were operated on between 1992 and 1995 Twelve patients underwent total cavopulmonary connection (TCPC) (fenestrated in 4), 9 had partial cavopulmonary connection (PCPC) and 7 had a palliative procedure. The pulmonary vascular structure was analyzed using morphometric techniques and was considered abnormal if wall abnormalities were noted: increase of medial thickness, intimal damage, muscular extension in more peripheral arteries than normal. There were 4 early deaths. The causes of death were hypertensive pulmonary crisis ( 2 patients-anomalous pulmonary venous return, mitral stenosis) and low cardiac output after TCPC ( 2 patients). The histologic findings were correlated against preoperative pulmonary arterial pressure (PAP), type of surgery and early outcome. In conclusion, (1) there is no strict correlation between hemodynamic and histological findings; (2) abnormal pulmonary vasculature may influence outcome and was always present when TCPC failed; (3) TCPC should not be performed in one stage but in two stages with lung biopsy at the first one.

\section{VIII-01}

Fetal echocardiographic screening of pregnant women with connective tissue disorders

\section{Gladman G, Silverman E, Laskin C, Bouttn C, Smallhorn J} The Hospital for Sick Children, Toronto, Canada

The risk of congenital complete heart block (CHB) in an infant born to a mother with antibody mediated connective tissue disease (CTD) has been estimated at 1:20. An increased incidence of structural cardiac anomalies has also been reported. To assess the role of fetal echocardiography in screening pregnant women known to possess the antibody markers of CTD, a total of 89 pregnancies in 78 women with known immunological markers of CTD were assessed by fetal echocardiography at 18,24 and 32 weeks gestation. Fetuses and babies presenting with $\mathrm{CHB}$ and anatomically normal hearts during the same study period, but without a maternal history of CTD, were also reviewed. Seventy-one women had clinical SLE or tested positive for antibody markers of the disease, 3 had Sjogren's syndrome and 4 mixed connective tissue disease. All were Ro or La antibody positive. No structurally abnormal fetal hearts were identified. One fetus developed $\mathrm{CHB}$ (died in utero). Five women had previously had a child with $\mathrm{CHB}$ - all produced a healthy infant subsequently without increasing pre-pregnancy maternal immunosuppression. Eight fetuses were diagnosed antenatally with $\mathrm{CHB}$ in mothers with no history of CTD (all these women subsequently proved to be antibody positive); 5/8 fetuses died. Similarly, 6 infants were born to morhers with no history of CTD and found unexpectedly at delivery to have $\mathrm{CHB}$ (again all mothers subsequently proved to be antibody positive); all survived. In conclusion, no structural fetal cardiac anomalies were identified. Of the 15 fetuses/neonates diagnosed with $\mathrm{CHB}$, only 1 was detected by screening; all 15 mothers eventually proved to be antibody positive. We did not observe an increased risk in subsequent pregnancies whose past obstetric history included an infant born with $\mathrm{CHB}$. Fetal echocardiography in addition to routine obstetric care may not be required to assess pregnant mothers with CTD.
VIII-02

Epidemiology of fetal congenital heart defects

Marek J, Skouranek J, Povysilova V

Kardiocentrum, University Hospital Motol, and Department of Pathology, Second Medical School, Charles Univerity, Prague, Czech Republic

The postnatal incidence of congenital heart defects (CHD) in Bohemia is $0.67 \%$. Anomalies were found in $2.1 \%$ of 3,962 stillborns $>28 \mathrm{wk}$ of gestation. In early pregnancy, the prevalence of cardiac lesions is higher. The most frequent anomaly was hypoplastic left heart (17.4\%), AVSD (12.4\%), DORV (11.6\%) and univentricular heart (11.2\%). What may cause these differences in pre- and postnatal prevalence of CHD? (1) Atrial communication and arterial duct are not prenatal CHD; (2) VSD is often missed on ECHO; (3) obstetrical ultrasonographers are not confident in identifying anomalies of the vessels; (4) obstetricians concentrate more on fetal investigations in the setting of a positive family history; (5) CHD can develop even after the 20th week of gestation; (6) the pre- and postnatal CHD spectrum is truly different. To assess the effectiveness of prenatal screening and the assumption of different $\mathrm{CHD}$ spectrum, the findings in prenatally diagnosed fetuses were compared with those expected to be born with "critical" CHD according to our previous epidemiological study. Of the 199,418 children born alive berween 1992 and 1994, 468 (2.3 per 1000 live-births) were assumed to be born with "critical" CHD. Children with PDA, ASD, VSD were not included; 151 with patent aretial duct, atrial and ventricular septal defects were excluded. Out of remaining 317 "critical" CHD, only 91 $(29 \%)$ were diagnosed prenatally. In hearts with single ventricle, and those with complete AV septal defect, the prenatal prevalence was higher than that assumed postnatally. $75 \%$ detection rate of double outlet right ventricle, $70 \%$ of critical aortic stenosis, $49 \%$ of hypoplastic left ventricle and $46 \%$ of Fallot's tetralogy was higher than in remaining other CHD's. This is in agreement with data published by Allan et al, demonstrating a different spectrum of prenatally diagnosed CHD, with high intrauterine death rate in some of them.

\section{VIII-03}

Mismatch between fetal diagnosis and surgical expectations Schade RP, van Venrooij FV, van de Wal HJCM, Bennink GBWE, Meijboom EJ

Pediatric Heart Center, Wilhelmina Children's Hospital, Utrecht, The Netherlands

One of the expectations of the prenatal establishment of congenital cardiac diagnosis is the improvement of postnatal care, specifically in regard to surgical treatment. Two populations of children with congenital heart disease were compared, originating from the same region between 1991-95. One consisted of 1794 patients referred to our fetal cardiology program for a level 3 scan. These referrals were based on maternal and/or fetal indications or an abnormal four-chamber view (44\%). The other consisted of 527 patients operated for structural heart disease. In our fetal cardiology program, 77 structural card iac mal formations were encountered, 13/77 were stillborn and 24/77 pregnancies were terminated - $15 / 24$ were terminated because of cardiac disease and associated chromosomal and/or organic disease, 9/24 for cardiac disease exclusively, diagnoses including hypoplastic left/right ventricle, double inlet left ventricle and tricuspid atresia. $40 / 77$ were live-born of which 12 required cardiac surgery, 4 neonatal (1 treated elsewhere). In the surgical group, 78/527 infants had surgery under the age of 1 month. Only 3 of these patients originated from the fetal program, 2 had a prenatal diagnosis of their malformation (pulmonary atresia $(\mathrm{PA})$ with ventricular septum defect (VSD), rhabdomyoma), 1 pt was considered normal prenatally but proved to have PA with VSD. 21/527 were operated within $72 \mathrm{hrs}$ after birth, $11 / 21$ had TGA, 6/21 pulmonary atresia. Of the $78 / 527$ pts, $80 \%$ must have undergone a level 1 or 2 prenatal echo (Dutch National Health Council), which means that optimally 62 patients could have been referred antenatally. Currently a serious mismatch exists between the two compared populations. To optimize surgical care one would like to have referred prenatally the missing of the $80 \%$ echoed patients. 
VIII-04

Atrial flutter in the fetus-presentation and follow-up

Notes

Benatar AA, Soyeur DJG, van Engelen AD, Brenner JI, Kleinmann CS, Stoutenbeek $P$, Meijboom EJ

University of Utrecht, Utrecht, The Netherlands, University of Liege,

Liege, Belgium, University of Maryland Medical Systems, Baltimore and Yale University, New Haven, United States of America

Fetal atrial flutter (AF) is a potentially lethal arrhythmia, and in utero management is difficult and controversial. The aim of this study was to evaluate fetal AF, the efficacy of maternally administered antiarrhythmic medication, postpartum management and outcome. A group of 32 fetuses with AF was retrospectively studied. Fetal hydrops were seen in 17 pts, 15 of which received maternal therapy; one was immediately delivered and one not treated on account of a severe nontreatable cardiac malformation. In the non-hydropic group of 14 patients, 8 were treated and the remaining 6 were delivered immediately. In the hydropic group, 13 received single drug therapy (digoxin), 3 multiple drugs. In the nonhydropic group, 6 received a single drug ( 5 digoxin and 1 sotalol) and 2 multiple. One patient with rapid 1:1 atrioventricular conduction (heart rate 480 per minute) died in utero. Of the 31 liveborn infants, 12 were in atrial flutter at birth. Electrical cardioversion was successful in 8 of 9 parients. Transvenous overdrive atrial pacing was successful in one of 2 patients. Follow-up ranges from 6 months to 10.25 years, median 5.2 years. No recurrences in atrial flutter have occurred beyond the neonatal period. In conclusion, fetal atrial flutter is a serious and lifethreatening rhythm disorder particularly when it causes hydrops. Treatment is aimed at controlling ventricular rate or preferably conversion to sinus rhythm. Digoxin was the drug of first choice in this study and was successful as single therapy in $68 \%$ of all treated cases. Class III agents require further evaluation. Once fetuses with $\mathrm{AF}$ survive, their future is good and prophylaxis for 6 months suffices.

VIII-05

Efficacy of flecainide versus digoxin in management of fetal supraventricular tachycardia

Frohn-Mulder IM, Stewart PA, Witsenburg M, den Hollander NS, Wladimiroff JW, Hess J

Department of Peduatrics, Division of Pediatric Cardiology, and Department

of Obstetrics and Gynecology, Division of Prenatal Diagnosts, Sophia

Children's Hospital, Unvversity Hospital Rotterdam, Rotterdam. The

Netherlands

Digoxin can be used successfully for treatment of fetal supraventricular tachycardia (SVT), but in cases of fetal hydrops the result is disappointing. This retrospective study was carried out to assess whether the introduction of flecainide altered management and outcome of fetal supraventricular tachycardia, especially in cases who presented with fetal hydrops. We retrospectively studied data of all patients that were referred because of fetal SVT from 1982-1993. There were 49 patients in the records of whom 14 were not treated prenatally, either because of intermittent tachycardia, or because of advanced pregnancy in which it was decided to induce labour to treat the SVT postnatally. Of the remaining 35 fetuses that were treated transplacentally, 22 presented without and 13 with hydrops. These groups differed significantly with respect to restoration of normal sinus rhythm (SR) (73 vs $30 \%$, $\mathrm{p}<0.001)$ and mortality ( 0 vs $46 \%, \mathrm{p}<0.001)$. Digoxin was effective in restoring SR in $55 \%$ of the non-hydropic fetuses but in only $8 \%$ of the hydropic fetuses. Flecainide was effective in restoring $S R$ in all nonhydropic fetuses after digoxin failure $(\mathrm{p}<0.05)$ and also in $43 \%$ of the hydropic fetuses. It significantly reduced mortality $(p<0.001)$. No adverse effects were seen. In conclusion, flecainide is superior to digoxin for transplacental treatment of fetal SVT, especially when hydrops is present. Introduction of flecainide reduced mortality significantly. 


\section{P-01}

Quantitation of subaortic stenosis caused by restrictive ventricular septal defect in double inlet left ventricle by transthoracic three-dimensional echocardiography

Vogel M, Ho SY, Anderson RH

Department of Paediatrics, German Heart Center, Berlin, Germany and National Heart and Lung Institute, London, United Kingdom

We had previously reported the accuracy of 3D-echo in measuring diameters of ventricular septal defects (VSD) in vitro. The purpose of this study was to examine the ability of 3D-echo to measure in life the area of defects and reconstruct its irregular contours in double inlet left ventricle with transposition and the aorta arising from the rudimentary $\mathrm{RV}$, in whom a restrictive defect frequently presents the clinical problem of subaortic stenosis. The measurement by $3 \mathrm{D}$-echo was compared to direct assessment in 6 specimens scanned in a water-bath. The VSD was filled with a silicone sealant, which was extracted to measure its narrowest area. Correlation between $3 \mathrm{D}$-echo and true area had an rvalue of 0.98 with limits for agreement reaching from -0.1 to $+0.08 \mathrm{~cm}^{2}$. 3D-Reconstruction revealed the irregular shape and contour which matched true VSD contours in the specimens. After validation of the technique, 9 patients, mean age 9.1 (2-15) years, including 3 having undergone surgical enlargement of VSD, were examined with transthoracic $3 \mathrm{D}$-echo. Cross-sections displaying the VSD and the aortic valve en face could be found in all datasets. The longest and shortest diamerer of the defect was $23.3 \pm 6.6$, and $15.5 \pm 3.5 \mathrm{~mm}$ respectively, and the area measured $6.4 \pm 1.1 \mathrm{~cm}^{2}$. The aortic diameter was $18 \pm 3.6 \mathrm{~mm}$, and the area $3 \pm 1 \mathrm{~cm}^{2}$. The ratio between VSD and aortic areas was $1.37(0.79$ 1.89), two-thirds of patients with ratio of areas $<1$ had subaortic stenosis confirmed by a Doppler gradient at rest. We conclude that 3Dechocardiography can accurately quantitate size of VSD and should be included in the evaluation of patients with double inlet LV.

\section{P-02}

Results of electrophysiologic testing after modified Fontan operation-implications of postoperative hemodynamics and the type of operation

Paul T, Schmidt KD, Bökenkamp R, Luhmer I, Ziemer G, Hecker H, Kallfelz $H C$

Pediatric Cardiology, Hannover Medical School, Hannover, Germany

Electrophysiological study (EPS) and assessment of postoperative hemodynamics were performed in 41 children after modified Fontan operation (atriopulmonary connection $n=15$, total cavopulmonary connection $n=26$; mean age at operation 6.2 years, mean interval operation-EPS 2.9 years). EPS consisted of evaluation of sinus node recovery time as well as programmed atrial and ventricular stimulation with double extra stimuli at 2 paced basic cycle lengths, respectively. Clinical postoperative dysrhythmias (ECG and Holter) were found in 10 patients: 6 children had significant sinus dysfunction; recurrent symptomatic episodes of atrial flutter (AFL) and supraventricular tachycardia (SVT) were documented in 2 patients each. During EPS, 13/41 parients had a physiologic result. Prolonged $(>350 \mathrm{msec})$ corrected sinus node recovery time was found in 11 patients, SVT was induced in 5 children. Twelve patients had inducible AFL, 1 patient had primary ventricular fibrillation induced. Patients with a pathologic EPS had a significantly $(\mathrm{p}<0.05)$ higher mean postoperative pulmonary artery pressure $(10.5 \pm 1.9 \mathrm{vs} 9.1 \pm 1.9 \mathrm{~mm} \mathrm{Hg})$ than those who had a normal EPS. All 6 patients who had a total cavopulmonary connection performed and a mean postoperative pulmonary artery pressure $<8.5$ $\mathrm{mm} \mathrm{Hg}$ had a normal EPS in comparison with the rest of the group $(p<0.05)$. Incidence of electrophysiologic abnormalities was high after modified Fontan operation. Pathologic results of EPS were associated with postoperative hemodynamics and the type of operation with more favorable results in parients with a total cavopulmonary connection.

\section{P-03}

Normal ranges of heart rate variability in childhood

Massin $M$, von Bernuth $G$

Department of Pediatric Cardiology, RWTH Aachen, Aachen, Germany

Heart rate variability (HRV) as determined from 24-hour Holter recordings is being recognized as a measure of cardiac autonomic control. However, there are few data on HRV in children and normal ranges are not yet available for the pediatric population. The present study examined HRV-indices in 210 healthy neonates, infants and children aged 3 days to 14 years. Five time-domain measures (standard deviation [SD] of all RR intervals over the length of the analysis; mean of the SD and SD of the means of all RR intervals for all 5-minute segments of the analysis; square root of the mean of the sum of squares of differences between adjacent RR intervals over the length of the analysis; and percentage of differences between adjacent $R R$ intervals that are greater than $50 \mathrm{msec}$ for the whole analysis) and 4 frequencydomain measures $(\mathrm{B} 1<0.004 \mathrm{~Hz} ; \mathrm{B} 2<0.004-0.04 \mathrm{~Hz} ; \mathrm{B} 3$ 0.04-0.15 $\mathrm{Hz}$; $\mathrm{B} 40.15-0.4 \mathrm{~Hz}$ ) were examined. The ratio of power in low/high frequency bands (division of B3/B4) or balance B3/B4, which is used as a measure of sympathovagal balance, was also calculated. Our data show a significant positive correlation between HRV-indices and the mean of all RR intervals over length of the analysis (linear function) and between $\mathrm{HRV}$-indices and age of the patients (power function). A negative correlation was found berween the balance $\mathrm{B} 3 / \mathrm{B} 4$ and the age of the patients and the mean of all RR intervals over 24 hours. The multiple correlation analysis confirmed the independent effect of age and mean of all RR intervals on the HRV. These data of HRV determinations in a healthy pediatric population confirmed a progressive maturation of the autonomic nervous system during childhood and may be utilized to examine the effects of underlying disease progress or therapeutic interventions on cardiac autonomic tone in children.

P-04

Myocardial viability and systolic function of the systemic right ventricle after Senning operation for transposition of the great arteries

Labbé L, Barat JL, Douard H, Chevalier L, Ducassou D, Broustet JP, Choussat $A$ Hôpital Haut-Lévêque, Pessac, France

After Senning operation for complete transposition of the great arteries (TGA), subclinical right ventricular dysfunction is often described. Preoperative or postoperative myocardial damage due to hypoxemia has been suspected. To detect abnormalities of right ventricular viability and their relationship with right ventricular function, we studied 30 asymptomatic patients ( 6 girls, 24 boys), $12.5 \pm 3$ years after Senning correction for TGA. The mean age at surgery was $8.8 \pm 5$ months. All patients performed myocardial tomoscintigraphy at rest, one hour after injection of $1.5 \mathrm{mCi}$ of Thallium 201. Right ventricular ejection fraction (RVEF) was measured by first-pass radionuclide angiocardiography $(99 \mathrm{mTe})$ at rest and at maximal exercise in the supine position. We found myocardial viability abnormalities of the systemic right ventricle in $14 / 30$ patients ( $46 \%$ ): moderate in 9 patients and severe in 5 patients. Resting RVEF and maximal end-exercise RVEF were significantly lower in patients with myocardial defects (respectively $44 \pm 5$ vs $51 \pm 7 \%, p<0.05$ and $48 \pm 9$ vs $58 \pm 9 \%, p<0.05$ ). No correlation was found between the age at the surgery and the occurrence of abnormalities of right ventricular myocardial viability. In conclusion, after Senning operation for TGA, myocardial defects of the systemic right ventricle are frequent, and are associated with altered right ventricular function at rest and at peak-exercise. 


\section{P-05}

Lung perfusion in bidirectional cavopulmonary anastomosis with pulsatile pulmonary blood flow-quantitative analysis using radionuclide angiocardiography

Reich O, Horváth P, Ruth C, Krejcir FM, Skouranek J

Kardiocentrum, University Hospital Motol, Prague, Czech Republic

A new non-invasive method for quantitative analysis of pulmonary perfusion in patients with bidirectional cavopulmonary anastomosis (BCPA) and sources of pulsatile blood flow (SPBF) is presented. The method enables quantification left-to-right lung flow ratio and relative contribution of BCPA and SPBF to perfusion of each lung. At the same time, cavo-caval collaterals may be diagnosed and ventricular function studied. In patients with BCPA, SPBF may improve pulmonary ventilation/perfusion ratio and prevent formation of pulmonary arteriovenousfistulae. ExcessiveSPBF increases preload of thesystemic ventricleand may facilitate cavo-caval collateral flow. Radionuclide angiocardiography was used for the quantitative analysis and for visualization of cavo-caval collaterals in 18 patients with BCPA and SPBF. In 8 patients (44\%), cavo-caval collaterals prevented the quantitative analysis. In 10 patients without cavo-caval collaterals, BCPA provided $42.3 \pm 3.4 \%$ (mean \pm SEM) of total pulmonary blood flow. From the total BCPA flow, $67.2 \pm 4.3 \%$ was directed to the ipsilateral lung. This lung received only $16.5 \pm 3.3 \%$ of all the blood from SPBF. The blood flow to the lung at the side of BCPA accounted for $35.3 \pm 1.7 \%$ of the toral pulmonary blood flow. Conclusions-Radionuclide angiocardiography allows the quantitative analysis of pulmonary blood supply in BCPA with SPBF except in patients with cavo-caval collaterals and/or bilateral BCPA; non-pulsatile flow from BCPA flow is mainly directed to the ipsilateral lung, whereas pulsatile flow to the contralateral lung; total perfusion of the ipsilateral lung is less than the perfusion of the contralateral lung.

P-06

Thermal balloon angioplasty of experimental arterial stenoses in lambs-a pilot study

Miller PA, Sreeram N, Townsend P, Morton DB

Heart Unit, Birmingham Children's Hospital and Boomedical Services Unit, University of Birmingham, Birmingham, United Kingdom

The aim of this study was to compare standard balloon dilation (SBA) with radiofrequency thermal balloon dilation (TBA) in an experimental model of arterial stenosis. Surgical stenoses were created in the right femoral artery of 12 neonatal lambs (median weight $7.6 \mathrm{~kg}$, range 6.1 to $11.5 \mathrm{~kg}$ ), with cutdown on the vessel under general anesthesia, and tying of an absorbable ( $5 / 0$ chromic catgut) suture to reduce the luminal diameter by $50 \%$. At 6 to $8 \mathrm{wks}$ after creation of the stenosis, the animals were catheterized by cannulation of the contralateral femoral artery Following angiography, balloon dilation of the stenosis was attempted using either a standard balloon or TBA. The PLOSA system (Boston Scientific) was used for TBA; in this system radiofrequency energy is used to heat the fluid in the balloon to an adjustable nominal temperature. Three lambs had complete occlusion of the femoral artery at the ligature site with extensive collateral vessels, and no dilations were performed. Of the remaining 9, SBA was performed in 4 cases using inflation pressures of up to 16 atmospheres, with successful outcome (abolition of waist) in 25\%. TBA was performed in the other 5 (inflation pressure 4 atmospheres, temperatures of $65-100^{\circ} \mathrm{C}$ for $60 \mathrm{sec}$ ). Inıtial success with complete disappearance of waist was achieved in $80 \%$. Two of the successfully treated lambs subsequently developed vascular complications and were terminated. The other 2 were followed-up by repeat angiography 4 to $6 \mathrm{wks}$ after initial TBA. In both cases the treated vessel was completely patent, with no residual waist or gradient, and equivalent absolute volume flow compared with the untreated artery. The early results suggest immediate loss of a waist and a greater increase of arterial cross-sectional area with low pressure TBA when compared with SBA.
P-07

Follow-up outcome of balloon angioplasty of recoarctation of the aorta Yetman A, Nykanen D, Sunnegardh J, McCrindle B, Freedom RM, Benson $L$ Hospital for Sick Children, Toronto, Canada

This retrospective review was undertaken to identify predictors of longterm hemodynamic outcome in a cohort of patients having undergone balloon angioplasty of postoperative recoarctation of the aorta. From January 1984 through January 1995, balloon angioplasty was performed in 99 patients with aortic recoarctation after surgical repair. Mean age at dilatation was $4.0 \pm 5.0$ (range $0.1-20.0$, median 1.1) years. Patients had a mean weight of $15.2 \pm 14.7$ (range $2.7-81.0$ ) kg. The mean systolic pressure gradient across the stenotic site was reduced from $37 \pm 24$ to $11 \pm 14 \mathrm{~mm} \mathrm{Hg}$ after dilatation ( $<<0.00001$ ). The mean diameter of the stenotic site, measured in frontal and lateral views at angiography, increased by $38 \pm 2.8$ and $35 \pm 3.0 \%$ respectively ( $<<0.001$ ). Neurologic events occurred in 2 patients with one death. Non-fatal aortic rupture occurred in 1 parient. Immediate successful results, defined as a post-procedure gradient of $<20 \mathrm{~mm} \mathrm{Hg}$, were obtanned in 87 patients. At long-term follow-up (median 4 yrs, range 2 mos- 10 yrs), 64 patients had gradients $<20 \mathrm{~mm} \mathrm{Hg}$ and were free from reintervention. Transverse arch hypoplasia (TAH), defined as a transverse arch dimension $<2$ SD of normal for age, was the only significant predictor of a suboptimal long-term outcome with $47 \%$ long-term success in patients with TAH vs $73 \%$ in patients with normal arch dimensions $(p=0.01)$. Percutaneous balloon angioplasty for recoarctation of the aorta is immediately effective in reducing pressure gradients. While the majority of patients with normal transverse arch dimensions will achieve longterm benefit, the incidence of recurrent stenosis remains higher in those patients with transverse arch hypoplasia.

P-08

Morbidity after modified Fontan operation-necessity of detailed clinical and laboratory evaluation

Kaulitz R, Luhmer I, Bergmann F, Kallfelz HC

Medical School Hannover, Hannover, Germany

Increasing long-term survival after MFO raises the question after sequelae even in clinically asymptomatic patients. We studied a group of 80 surviving parients who underwent a modified Fontan operation for various forms of functionally univentricular hearts (15 pts after atriopulmonary anastomosis, 65 pts after total cavopulmonary anastomosis). Follow-up ranged from 16 110 months. Atrial dysrhythmia were found on Holter in $17 \%$, with the need for antiarrhythmic treatment in $10 \mathrm{pts}$. Bicycle exercise testing was feasible in $26 \mathrm{pts}$ and revealed normal exercise capacity in $31 \%$. Symptomatic proteinlosing enteropathy was found in $2 \mathrm{pts}$. Partial atrial thrombosis was found on echocardiogram in 4 pts during the early $(3 \mathrm{pts})$ and intermediate $(1 \mathrm{pt})$ postoperative period. With a mean postoperative interval of 32 months, 62 ptsunderwentcardiaccatheterization (mean systemic venous pressure ranged from $6-19$, mean $11 \mathrm{~mm} \mathrm{Hg}$ ); cardiac index of less than $31 / \mathrm{min} / \mathrm{m}^{2}$ was calculated in $44 \%$. The laboratory invesrigation as performed in $50 \mathrm{pts}$ considered various aspects of postoperative problems. This revealed normal values for liver enzymes, AT IIl, plasminogen and protein $S$, creatincinase, vitamin $A$ and $E$. Abnormal values were mainly documented for $y G T$ and bilirubin (in 34\%). Hypoproteinemia was found in 31\%; all of these 15 pts had undergone a total cavopulmonary anastomosis, 9 of them had follow-up less than 3 yrs. Coagulation factor analysis revealed protein $\mathrm{C}$ deficiency in 4 (10\%); 3 other pts had laboratory signs of activated coagulation as tested by TAT-, FBDP, F1+2-level. Abnormal findings were not related to postoperative interval, systemic venous pressure or cardiac index. Even in clinically asymptomatic pts, a high percentage of pts with reduced exercise capacity, impaired $\mathrm{CI}$ and abnormal laboratory findings were discovered on detailed evaluation of intermediate follow-up. 


\section{P-09}

Value of autopsy in paediatric cardiology

Gatzoulis $M A$, Sheppard $M$, Ho SY

Royal Brompton Hospital and National Heart \& Lung Institute, London, United Kingdom

The aim of this study was to assess the current value of autopsy in paediatric cardiology and cardiothoracic surgery and determine its potential impact on clinical practice. Data on all our paediatric patients with congenital or acquired heart disease who died from a cardiac cause between January 1992 and July 1995 were obtained from reviewing the hospital files, autopsy records and specimens, and audit reports. The value of autopsy was assessed according to its contribution in establishing the precise cause of death (confirmed, clarified, or uncertain) and the anatomy (simple confirmation or additional information provided). For cases not submitted to autopsy, the clinical information relating to the cause of death was assessed and the case assigned as cause of death firm, uncertain, or unknown. 106 cases were identified terminating in death (61 males, age: 1 day-20 years). 70 cases were early ( $<1$ month) hospital postoperative deaths. The rest were either cases who had previously undergone surgery or purely medical ones. Autopsy was performed in 59 of the 106 cases $(55.6 \%)$. The precise cause of death was confirmed in $33(55.9 \%)$, clarified in $22(37.3 \%)$ and remained uncertain in $4(7.8 \%)$. Additional information regarding the anatomy was found in $8(13.6 \%)$ cases. In 5 cases $(8.5 \%)$, the autopsy detected findings, which, if known prior to death, would probably have improved outcome. For the patients dying without an autopsy, the cause of death remained uncertain in $10(21.3 \%)$ and unknown in $7(14.9 \%)$. In $36.2 \%$ of cases, therefore, a firm cause of death that might have been provided by an autopsy was missing. Autopsy in paediatric cardiology continues to provide clinically relevant information at a high level. It remains vital for ensuring quality of medical care, inspiring improvements in future management, and increasingour understanding of congenital heart disease and, should therefore, be actively sought in all cases.

P-10

Quantitative analysis of signal-averaged electrocardiographic parameters in healthy children

Gehrmann J, Kecectoglu D, Kehl HG, Muller F, Fetsch T, Hellmich M, Vogt J

Westfalische Wilhelms-Universität, Münster, Germany

The noninvasive technique of signal-averaged electrocardiography (SAECG) may provide useful prognostic information in children with congenital heart disease and cardiomyopathy at risk for ventricular tachycardia. The aim of this study was to establish reference values in a heal thy pediatric population. The study group consisted of 100 healthy children ( 52 female and 48 male; mean age $10.4 \pm 3.9$ years, range 2.7 to 16.5). The SAECGs were recorded during sinus rhythm from standard bipolar orthogonal X, Yand Z leads and a mean of 155 cycles with a noise level less than $0.9 \mu \mathrm{V}$ were analyzed. The signals were amplified, averaged and filtered with a digital bidirectional band pass filter at cutoff frequencies of $40-250 \mathrm{~Hz}$. The duration of the signal-averaged filtered QRS (QRSd) and of the low-amplitude signals in the terminal portion of the QRS complex less than $40 \mu \mathrm{V}(\mathrm{L} . \mathrm{AS}<40)$, and the root-meansquare voltage in the last $40 \mathrm{~ms}$ of the filtered QRS (RMS-40) were measured and expressed as mean values $\pm S D$. For the total group $Q R S d$ was $84.9 \pm 9.2 \mathrm{~ms}, \mathrm{LAS}<4017.3 \pm 6.3 \mathrm{~ms}$, RMS-40 109.3 $\pm 68.9 \mu \mathrm{V}$. The age group of 3-7 years had significantly shorter QRSd $(78 \mathrm{~ms})$ than the groups of $7-12$ years $(86.2 \mathrm{~ms})$ and of $12-16$ years $(88.5 \mathrm{~ms})(\mathrm{p}<0.001)$. No significant differences between boys and girls were found for QRSd, for LAS $<40$, and for RMS-40. Confidencelimits of $95 \%$ for QRSd were $\leq 103 \mathrm{~ms}$, for $\mathrm{LAS}<40 \leq 30 \mathrm{~ms}$ and for RMS $-40 \leq 247 \mu \mathrm{V}$. In conclusion, these confidence limits and their age-dependency should be considered when SAECG is performed to identify children at risk for ventricular tachycardia. For RMS-40, no reasonable cutoff values could be determined.
P-11

Diagnosis and outcome of fetal cardiomyopathies

Guirgis N-M, Casasoprana A, Magnier S, Kchouk H, Blot P, Azancot A Robert Debré Hospital, Parts, France

The aim of this retrospective study was to review the etiology and the outcome of fetal cardiomyopathies (FCM) diagnosed by fetal echocardiography (FE) after exclusion of right or left heart obstructions and ductus arteriosus constriction. Between 1989 and 1995, 23 FCM were diagnosed by FE and classified into: 1) Hypertrophic FCM ( $n=18)$ metabolic $(\mathrm{n}=2)-1$ beta-lipase deficiency, 1 cytochromeoxidase deficiency diagnosed by amniocentesis and muscle biopsy [Neonatal death occurred]; twin-to-twin transfusion ( $\mathrm{n}=4$ ), involving the recipient twin, with postnatal regression, with 1 case followed for pulmonary stenosis; intrauterine growth retardation $(n=1)$, which proved postnatally to be Rubinstein-Taybi Syndrome and followed regularly since 6 years; maternal diabetes $(n=10)$ with postnatal progressive regression; one case considered as idiopathic, proved to have postnatal, peripheral pulmonary stenosis; the child is alive with 5 years follow-up. 2) Dilated FCM $(n=5)$ : dilated left ventricular cardiomyopathy associated with fetal supraventricular tachycardia (SVT) $(\mathrm{n}=2)$ (Regression occurred in both cases after arrhythmia control; one died from prematurity, the other is alive, with 5 years follow-up); dilated idiopathic right ventricular cardiomyopathy $(n=1)$ with neonatal death; endocardial fibroelastosis $(n=2)$ (family chose termination of pregnancy). We conclude that 1 ) Unexplained fetal hypertrophy or dilatarion may be a part of a generalized systemic disorder. 2) Screening is indicated to identify secondary causes of FCM, specially those potentially reversible. 3) Regression of dilated FCM associated with SVT occurs after arrhythmia control. 4) FCM secondary to peripheral pulmonary stenosis may be difficult to diagnose prenatally, as fetal pulmonary blood flow is relatively low.

P-12

Surgical results in atrioventricular septal defects with and without Down syndrome

Halees Z, Borgmann I, de Moor M, Fadley F, Galal $O$

King Fasal Specialist Hospital and Research Center, Riyadh, Saudi Arabia

The aim of the study was to examine whether there is a difference in mortality between patients with atrioventricular septal defects (AVSD) with and withour Down syndrome. Between March 1988 and July 1994, 83 patients (pts) with complete balanced AVSD underwent surgery at our institution. Chromosomal analysis revealed Down syndrome in 63 pts $(75.9 \%)$. Age at surgery of the Down pts was 21.7 months and 31 months in the non-Down group. Cardiac catheterisation was performed in 58 Down pts and 13 non-Down pts. The mean pulmonary artery (PA) pressure in the Down pts was $48.3 \mathrm{~mm} \mathrm{Hg}$, and $39.7 \mathrm{~mm} \mathrm{Hg}$ in the non-Down pts. The left-sided atrioventricular valve (AV) was competent (by echo) in 17/63 Down pts and in 9/20 nonDown pts. The right-sided atrioventricular valve (AV) was competent in 20/63 Down pts and in 10/20 non-Down pts. According to the size and distribution of the septal defects, a one-patch or a double-patch technique was used. Small VSDs (6/63 Down pts and 9/20 non-Down pts) could be closed directly. In all Down pts, both AV valves were repaired; in two pts, the left-sided $A V$ valve was not repaired and valve replacement was done. In 6/20 non-Down pts, both AV valves had to be repaired. In another patient, valve replacement was necessary. In two children, solely the right AV valvar component needed repair. 11/63 (17.5\%) Down pts and 2/20 (10\%) non-Down pts died during the hospital stay. AV valve replacement in Down prs was associated with a very high mortality. Our results show that pts with Down syndrome have a higher mortality than non-Down pts. The reason could be the higher PA pressure. 


\section{P-13}

Recurrent sustained monomorphic ventricular tachycardias in patients with tetralogy of Fallot late after surgical repair-feasibility for radiofrequency current treatment?

Hebe J, Weiß C, Siebels J, Kuck K-H

Department of Cardiology, St. Georg Hospital and University Hospital Eppendorf, Hamburg, Germany

Out of 32 patients (pts) with a congenital heart defect (CHD) who underwent percutaneous radiofrequency current (RFC) treatment of tachyarrhythmias in our institution, 3 pts presented with recurrent episodes ventricular tachycardia (VT) 4 mo to $17 \mathrm{yrs}$ after surgical repair of tetralogy of Fallot (TOF). For repair of the right ventricular outflow tract (RVOT), an infundibular resection was performed in 2 pts; a transannular RVOT-parch was required in the remaining. A transatrial approach was used in 2 pts. VT-related symptoms ranged from palpitations to episodes of dizziness or syncope. Antiarrhythmic regimen failed to control recurrence of VT. In all pts, ECG recordings showed VT generating from the RVOT. During electrophysiologic study a macro-reentrant circuit was the cause of VT in all 3 pts with an area of slow conduction between the ventricular aspect of the pulmonary valve annulus and the superior border of a scar resulting from previous infundibulotomy at the right anterior free wall. Conversely, in the pt who underwent a transannular patch technique, the area of slow conduction was found in the septal portion of the RVOT, along the septal attachment of the patch. With a median of 7 RFC-applications, all 3 pts were cured from VT. The session duration ranged from 2.5 to 8.0 hours, fluoroscopy was required for 12.2 to 38.6 minutes. During a follow-up of 1.2 to 3.8 years, all prs were free of sustained VT, there was no need for any further antiarrhythmic treatment. In conclusion, scar tissue secondary to surgical repair of RVOT obstruction in TOF may give rise to monomorphic reentrant ventricular tachycardia. RF current treatment of this arrhythmogenic substrate in TOF patients is feasible and should be recommended as a first line therapy.

\section{P-14}

Sox-4 gene is required for cardiac outflow tract formation

Schilham MW, Oosterwegel MA, Moerer P, Verbeek S, Lamers WH, Meijboom EJ, Kruisbeek AM, Cumano A, Clevers $H$

Departments of Immunology, Transgenic Mouse Facility and Pediatric Cardiology, University of Utrecht, Utrecht and the Department of Anatomy and Embryology, Academic Medical Center, University of Amsterdam, Amsterdam, The Netherlands

During murine embryogenesis, the transcriptional activator Sox- 4 is expressed at multiple sites. To determine its essential functions, a gene disruption experiment was performed. In addition to a defect in the proB lymphocyte expansion, in all Sox-4 ${ }^{1 /}$ embryos, generalized edema developed leading to circulatory failure and death at day E1 4 . Histologi$\mathrm{cal}$ analysis consistently revealed malformation of the arterial pole accompanied by a large malalignment ventricular septum defect. This resulted from impaired development of the endocardial cushion tissue (a specific site of Sox- 4 expression) into the semilunar valves and the outlet portion of the muscular ventricular septum. The resulting developmental defects are reminiscent of congenital conotruncal anomalies in man usually associated with a spectrum of congenital heart defects including tetralogy of Fallot, double outlet right ventricle and common arterial trunk. These types of congenital cardiac defects are thought to be caused by a developmental arrest of the leftward expansion of the embryonic outflow tract, resulting in a failure of the connection of the outflow tract with the left side of the heart. In conclusion, the chromosome 6 Sox- 4 gene plays an important role in the cardiac outflow tract formation and in the pro- $\mathrm{B}$ lymphocyte expansion of mice. Although the cardiac expression resembles that of the DiGeorge syndrome, it is genetically not at all related to this syndrome. It is of crucial importance to establish the role and eventual impact on the formation of the heart of the Sox- 4 gene in the human situation.
P-15

Occlusion of congenital ventricular septal defects by the buttoned device Stderis EB, Walsh KP, Haddad IL, Chen C-R, Kulkarni $H$ Athentan Institute of Pediatric Cardiology, Athens, Greece

Congenital ventricular septal defect (VSD) occlusion was performed in 18 patients using the buttoned device (DEV) since March 1994. The device was used directly or over a wire (OW), through 7-9 Fr sheaths. Fifteen of the defects were perimembranous (MEM) and 3 were muscular (MUS).

$\begin{array}{lrccrcc} & \text { N } & \text { AGE (ns) } & \text { DEV }(\mathrm{mm}) & \text { FO } & \text { PO } & \text { COMPL } \\ \text { MEM } & 15 & 4-35 & 15-35 & 11 & 4 & 2 \\ \text { MUS } & 3 & 7-14 & 20-25 & 2 & 1 & -\end{array}$

All MUS defects were corrected using the right jugular vein; all MEM defects were corrected using the femoral vein; in the last 12 VSDs the OW technique was used. Qp:Qs exceeded 1.5:1 in all cases. Aneurysm of the membranous septum was seen in all MEM VSDs. Thirteen VSDs were fully occluded (FO) and 5 had trivial residual shunts (PO). Two MEM VSDs were complicated (COMPL) by mild aortic insufficiency. One patient developed transient complete heart block: no tricuspid insufficiency was noticed. A MEM defect was considered appropriate for occlusion if the distance from the center of the defect to the aortic valve leaflet was more than $50 \%$ of the size of the DEV required. In conclusion, VSD occlusion was feasible, safe and effective in most attempted cases including MEM VSDs; proper case and DEV size selection is necessary.

P-16

Pulmonary function abnormalities after the Fontan operation Vignati G, Corato A, Banfi F, Sacerdoti C, Austoni P, Mauri L, Figini A Pediatric Cardiology, Niguarda Hospital, Milan. Italy

The abnormal adaptation to exercise after the Fontan operation is primarily due to a suboptimal increase in cardiac output. We postulated that abnormal pulmonary function could contribute to reducing effort tolerance on these patients (pts). Eleven pts (mean age $15.7 \pm 7.5 \mathrm{yrs}$ ) previously underwent a Fontan operation who returned in the first 6 months of 1995 to our unit for follow-up were studied. The mean postoperative follow-up was $5.8 \pm 2$ yrs. An atropulmonary connection was performed in 9 pts and a bicaval anastomosis in 3 . Nine pts were asymptomatic, while 2 had mild effort dyspnea. Basal ejection fraction was normal in 9 prs and mildly depressed in 2 . Basal cardiac index was $1.8 \pm 2.31 / \mathrm{min} / \mathrm{m}^{2}$ and increased to $4.8 \pm 0.5 \mathrm{l} / \mathrm{min} / \mathrm{m}^{2}$ during exercise. Effort tolerance was $72 \pm 10 \%$ of predicted values, with maximal $O$ consumption of $59 \pm 13 \%$ of predicted. Basal spirometry showed a mild restrictive pattern in $7 \mathrm{pts}$ with a decrease in total pulmonary capacity and in forced vital capacity, otherwise respiratory reserve (VE/MVV) was normal in all pts. Monoxid diffusion was impaired in all. $\mathrm{O}_{2}$ saturation near normal in basal condition decreased during exercise. The $\mathrm{VE} / \mathrm{VO}_{2}$ and $\mathrm{Vd} / \mathrm{Vt}$ were both abnormally increased. Lung scanning was abnormal in $7 \mathrm{pts}$, with defects of perfusion localized prevalently in left lung (6/7), especially in upper lobe (5/7). In conclusion, after the Fontan operation, pulmonary function is abnormal and can contribute in reducing effort tolerance. The mild pulmonary restrictive pattern is probably due to the complex surgical history of these pts and apparently does not affect the respiratory reserve. On the contrary, there is an Important impairment of gas diffusion, probably due to ventilation/ perfuston mismatch and/or to a damage of the alvelo-capillary membrane. As a consequence there is an increase in the minute ventilation and a decrease in the fraction of ventilation useful for gas exchange. 
P-17

Morbidity associated with Blalock-Taussig shunts and tetralogy of Fallot Gladman G, McCrindle B, Williams WG, Freedom RM, Benson LN The Hospital for Sick Children, Toronto, Canada

Considered a low risk management option for early palliation in tetralogy of Fallot(T/F), the morbidityassociated witha Blalock-Taussigshunt(BTS) can significantly impact on patient care. We analyzed the outcome for such surgery in the 1990s. Between 1/90 and 12/94, 65 infants (37 M) with T/F received a modified BTS. 60/65 with follow-up angiography wereassessed for clinical outcomes and shunt related morbidity and mortality. 68 randomly selected children who underwent angiography and repair of $\mathrm{T} / \mathrm{F}$ during the same period but without palliation were assessed for comparison. A right aortic arch was seen in $29 \%$ of parients in both groups. The BTS was rightsided in $95 \%$ of cases $(5 \mathrm{~mm}-80 \%, 4 \mathrm{~mm}-20 \%)$. Neonatal shunts accounted for 26/65 and median age at the time of BTS was 58 (range 1-440) days and median weight was 4.2 (range 1.3-8.8) kg. Average palliation time was 1.42 (range $0.20-3.22$ ) yrs. Self-limited morbidity complicated $11 \%$ of shunt operations. Distal (D) and proximal (P), left (LPA) and right (RPA) pulmonary artery diameters, indexed (I) to descending aortic diameter were:

\begin{tabular}{lcccc}
\multicolumn{1}{c}{ Group } & PRPAI & DRPAI & PLPAI & DLPAI \\
Single RBTS & $0.98(019)$ & $0.97(0.29)$ & $099(023)$ & $1.03(0.23)$ \\
Controls & $1.05(0.22)$ & $1.20(0.22)$ & $103(027)$ & $1.14(0.19)$ \\
& NS & p $<0.0001$ & NS & $p<0.01$
\end{tabular}

Moderate/severe angiographic RPA distortion occurred in 33\% of patients palliated with a single right $B T S$ vs $1 \%$ of controls $(p<0.0005)$ and occurred more frequently in neonatal compared to later palliation. BTS occlusion resulted in 1 death. Excluding non-cardiaccauses of death, survival in the BTS group was 90 vs $97 \%$ in controls. Theoverall results of palliation remain good. However, BTS are associared with significant PA abnormalities, especially when performed in the neonatal period and possibly alternative treatment regimes are more appropriate for this age group.

\section{P-18}

Is the open mitral "cleft" responsible for late mitral valve incompetence after correction of atrioventricular septal defects?

Alexi-Meskhishvili V, Schulze-Neick I, Hetzer R, Lange PE

Deutsches Herzzentrum Berlin, Berlin, Germany

Severe mitral valve incompetence (MVI) is the primary cause for reoperation after correction of the different types of AVSD. To determine major causes of late MVI, we analyzed our experience with the reoperations in patients (pts) with AVSD. Sixteen pts aged 2.5 months to 39 years were operated for severe (grade III-IV) MVI developing after correction of complete $(n=3)$, incomplete $(n=4)$ or intermediate $(n=5)$ types of AVSD. The interval between initial operation and reoperation was 2 weeks to 25 years (mean 4 years). Down's syndrome was present in 6 pts. During reoperation for severe MVI, a completely or partially open "cleft" was found in all 16 pts, combined with perforation at the "cleft" surure site in 4 (all operated at infancy), and dehiscence of the anterior mitral leaflet from a ventricular septal path in one pt. Complete "cleft" closure during reoperation combined with anuloplasty in 4 pts resulted in disappearance of MVI in $8 \mathrm{pts}$; in 4 pts mild MVI persisted. In 2 pts mitral valve replacement was necessary 7-14 days later after mitral valve reconstruction. There were no deaths or necessity of reoperation for MVI in all 16 pts early or late after discharge from hospital. In conclusion, MVI after correction of all types of AVSD is usually the result of incomplete closure, nonclosure, or separation of a previously sutured "cleft." In the absence of severely dysplastic or severely deformed mitral valve, complete closure of the "cleft," combined in some cases with anuloplasty, resulted in significant improvement of mitral valve function. To prevent perforation of the mitral valve at the "cleft" suture side, reinforcement of the "cleft" sutures with pericardial patches may be recommended in infants during the initial operation for AVSD.

\section{P-19}

Right ventricular diastolic function in children with pulmonary regurgitation after repair of tetralogy of Fallot-volumetric evaluation by magnetic resonance velocity mapping

Helbing WA, Niezen AR, Le Cessie S, van der Geest RJ, Meinesz S, Ottenkamp J, de Roos $A$

Unversity Hospital, Leiden, The Netherlands

Pulmonary regurgitation (PR) is an important problem in operated tetralogy of Fallot. Few volumetric data exist on the effects of PR on right ventricular (RV) diastolic function. The aim of the study was to assess RV diastolic function with $P R$, using RV time-volume curves generated by magnetic resonance velocity mapping (MR-VM). Nineteen children operated for TOF and 12 healthy children were studied. RV timevolume curves were reconstructed by summation of MR-VM pulmonary and tricuspid time-volume curves, using retrospective gating and 30 timeframes. Biventricular size and mass were assessed with multisection multiphase gradient-echo (GE) MR imaging. Graded exercise testing was performed. MR-VM results were compared to GE-MRI and echoDoppler. Systematic errors between GE-MR stroke volumes and MRVM flow measurements $(0.4-3 \mathrm{ml} /$ beat, $r=0.93-0.98)$ were small. Differences between MR-VM and Doppler time-to-peak E and A and E/A ratios were not statistically significant. In 13 patients (group I), late diastolic pulmonary artery forward flow contributed $1-14 \%$ to RV stroke volume; in 6 this pattern was absent (group II). In multivarıate analysis, significant differences with controls were observed for peak filling rare (increased-group I), filling fraction (decreased) and deceleration time (prolonged for group I and II, and for group II vs I). PR, exercise function (decreased vs controls) and biventricular size and function did not differ between patient groups. In conclusion, restriction to filling and impaired relaxation affect diastolic RV function in children with TOF and pulmonary regurgitation. With pulmonary regurgitation, RV time-volume curves can be generated by summation of pulmonary and tricuspid MR velocity mapping curves.

\section{P-20}

$\mathrm{N}$-terminal proatrial natriuretic factor in children with congenital heart disease

Holmstrom H, Hall C, Stokke O, Lindberg H, SorlandS, Thaulow E Pediatric Cardiology Unit, Institute for Surgical Research, Institute of Clinical Biochemistry, Thoracic and Cardiovascular Surgery Unit, National Hospital, Oslo, Norway

An objective and simple method for establishing and grading the diagnosis of heart failure in children is strongly needed. The $\mathrm{N}$-terminal part of the ANF prohormone, called proANF, has proven to be a clinically useful marker of heart failure in adults. We measured proANF in 62 children with congenital heart disease and in 62 healthy controls, in order to examine proANF's relation to different clinical and hemodynamic parameters. Doppler echocardiography was performed in all children, and 29 children also underwent cardiac catheterization. In pediatric patients without cardiac or renal disease, median proANF was $354 \mathrm{pmo}^{\circ-1}{ }^{-1}$. In children with congenital heart disease, the three groups with the highest proANF levels were children with documented high arrial pressure (median proANF $1760 \mathrm{pmol}^{-1} \mathrm{H}^{-1}$ ), a large left-to-right shunt (median proANF 1565 pmol. $\mathrm{l}^{-1}$ ) and moderate or severe heart

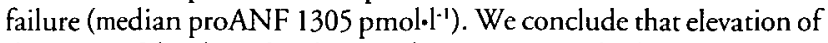
the proANF level is related to atrial pressures, to a high pulmonary to systemic flow ratio and signs of heart failure. Because of its stable and high basal plasma concentration and ease of measurement, proANF has a potential as a diagnostic tool in heart disease in children. 


\section{P-21}

Pacemakers for congenital heart disease in adults

Roos-Hesselink JW, van Reine RP, Spitaels SEC

Erasmus University, Rotterdam, The Netherlands

In 44 adults (age 18-83 years, mean 35.8) with pacemaker(PM) implantation for congenital heart disease, the incidence of brady-tachyarrhythmias was studied in relation to the PM-system.Ageat PM implantarion was 25.7 (range 4-73) yrs and during 10 years of follow-up an average of 2.3 (range 1-7) PM were implanted.

$\begin{array}{lcccc}\text { Indication for PM } & \begin{array}{c}\text { Congerital } \\ \text { block }\end{array} & \begin{array}{c}\text { Postoperative third } \\ \text { degree block }\end{array} & \begin{array}{c}\text { Sick } \\ \text { sinus }\end{array} & \begin{array}{c}\text { Brady-rachy } \\ \text { arrhythmias }\end{array} \\ \text { All partents } & 13 & 4 & 8 & 19 \\ \text { Congenital block } & 13 & - & - & - \\ \text { Transpostion } & - & - & 4 & 5 \\ \text { Terralogy of Fallor } & - & 1 & 1 & 4 \\ \text { Atrial septal defect } & - & - & 2 & 9 \\ \text { Ventricular septal defect } & - & 3 & - & 1 \\ \text { Pulmonary steriosts } & - & - & \text { I } & -\end{array}$

Of the 19 ptswith brady-and tachyarrhythmias, 13 received a ventricularlead only (VVI(-R)) and 6 had an atrial lead (4xDDD and 2xAAI). Of these 13 VVI patents, 10 had ongoing tachyarrhythmias, whereas only 1 of 6 pts with an atrial lead had tachyarrhythmias after PM implantation, $\mathrm{p}=0.04$.

$\begin{array}{lcc}\text { Complications } & \text { Epıcardal (\%) } & \text { Endocardial (\%) } \\ \text { Pneumothorax } & 0 & 8.3 \\ \text { Decubirus } & 40 & 10.4 \\ \text { Thrombosis } & 0 & 63 \\ \text { Infecuon } & 0 & 83 \\ \text { Lead fracture } & 40 & 21 \\ \text { Lead dislocation } & 0 & 8.3\end{array}$

Lead replacement was necessary n 15 prs after a mean of 8.7 (range 1-18) yrs in epicardial and after 5.3 (range 1-12) yrsin endocardial ventricularleads. The mean follow-up in the pts with only one ventricular lead until now was 12.7 (range 1-17) yrs in the epicardial and 6.9 (range 1-18) yrs in the endocardial group. These results favor the epicardial approach. Furthermore, in pts with brady-tachyarrhythmias, an AAI or DDD pacemaker is advisable for preventing arrial tachyarrhythmias.

\section{P-22}

Vascular wall composition after implantation of perforated versus nonperforated covered stents in a model of intimal hyperplasia Radtke W, Cowart K, Heisterkamp H, Beck C South Carolina Children's Heart Center, Charleston, United States of America We implanted 7 polyurethane (PU) laminated and 7 polycarbonate (PC) covered self-expanding Wallstents ( $5 \mathrm{~mm}$ diameter, $18 \mathrm{~mm}$ long) in the ductus arteriosus of 14 newborn lambs as a model of nonatheromarous intimal hyperplasia. Six newborn lambs had only balloon dilatation. Heparin and dextran were given during placement and lowdose aspirin during follow-up. The stented ductus was harvested after 3 , 6 and 15 weeks. $75 \mu \mathrm{m}$ sections were mounted and stained with the stent in place. Neo-intima, intima and media were measured from crosssections around the circumference, resulting in 30-35 measurements per cross-section. Fibroblasts migrated through the woven PC cover without significant foreign body reaction and formed neo-intima with increasing thickness to $311 \pm 19 \mu \mathrm{m}$ at 15 weeks. In PU laminated stents, slit-like blood filled spaces were seen in the native intima surrounding the stent. Perforations in the PU lamination led to transmigration of fibroblasts and neo-intima formation with equal thickness as in PC stents. In PU stents, the interface between cover and native intima was relatively acellular. Intima thickness increased in controls from $48 \mu \mathrm{m}$ to $751 \mu \mathrm{m}$ at 6 weeks and remained unchanged $(30 \%$ of total wall thickness). Media increased from 669 to $1790 \mu \mathrm{m}$ at 6 weeks and remained unchanged ( $70 \%$ of total wall thickness). After covered wall stent implantation, native intima thickness remained at $7 \%$ of total wall thickness. Media thickness increased to $1.34 \mathrm{~mm}$ at 6 weeks and dropped to $0.95 \mathrm{~mm}$ after 15 weeks. With both stents, the media occupied $92 \%$ of native wall thickness through the 15-week follow-up. Native intima and media thickness were equal in nonperforated and perforated covered stents. Media atrophy was minimal and only perforated PC covered stents accumulated neo-intima.
P-23

Effects of balloon pulmonary valvuloplasty on size of pulmonary annulus and arteries in tetralogy of Fallot

Godart F, Rey C, Mulwijk C, Brevière GM, Vaksmann G, Francart $C$ Department of Paediatric Cardiology, Lille, France

Balloon dilatation of the pulmonary valve was performed in 25 children with tetralogy of Fallot because of increasing cyanosis. Age and weight at the time of dilatation ranged from 0.5 to 10.5 (mean $3.5 \pm 2.6$ ) months and from 2.8 to 6.4 (mean $4.7 \pm 1.2$ ) kg. No procedure-related complications occurred. After dilatation, systemic oxygen saturation increased from a mean value of 76 to $85 \%(p=0.004)$. Despite this increase, 4 patients required Blalock-Taussig shunt soon after dilatation. The diameters of the pulmonary valve annulus and right and left pulmonary arteries were measured angiographically before dilatation and at a second catheterization or surgical repair performed at $9 \pm 5.6$ months of age. The pulmonary valve annulus increased from $6.09 \pm 1.17$ to $8.66 \pm 1.22$ $\mathrm{mm}(\mathrm{p}=0.01)$; the diamerer of the right pulmonary artery from $4.52 \pm 1.11$ to $6.54 \pm 1.11 \mathrm{~mm}(\mathrm{p}=0.01)$ and the diameter of the left pulmonary artery from $4.67 \pm 1.45$ to $6.46 \pm 1.7 \mathrm{~mm}(\mathrm{p}=0.04)$. Expressed as a mean $Z$ score, the annulus increased from $-5.2 \pm 3.3$ to $-0.334 .7(\mathrm{p}=0.03)$, the right pulmonary artery from $-3.02 \pm 0.76$ to $-1.6 \pm 1.46(p=0.028)$ and the left pulmonary artery from $-2.93 \pm 1.03$ to $-1.55 \pm 1.78(\mathrm{p}=0.04)$. Corrective surgery was performed in 21 patients at a mean age of $11.3 \pm 9.5$ months. The pulmonary annulus was considered large enough to avoid a transannular patch in $52 \%$ of patients. Damage of the right ventricular outflow tract was noticed in one parient. In conclusion, pulmonary valve dilatation can be performed in children with tetralogy of Fallot with no significant complications. It is associated with a subsequent increase in size of the pulmonary valve an nulus and the pulmonary arteries, leading to a reduction in need for transannular patch at the time of corrective surgery.

\section{P-24}

Atrioventricular septal defect associated with double outlet right ventricle

Helees $Z$, Moussa S, Galal O

King Faisal Specialust Hospital and Research Center, Riyadh, Saudn Arabia

The combination of atrioventricular septal defect (AVSD) with double outlet right ventricle (DORV) or tetralogy of Fallot (TOF) is a rare malformation. Although prom ising results have been obtained recently, the operative mortality to surgical treatment of this complex association remains high. In this study, we reviewed our experience concerning the underlying morphology of the disease and perioperative morbitity and mortality at our institution. Between 1983 and 1994, 15 pts with combined AVSD and DORV (11) or TOF (4) underwent total repair at our institution. Eleven had large ASD, two had ASD with small VSD component, and two had small ASD and large VSD. All pts had a single atrioventricular valve. Surgical repair was performed in all pts by patching the VSD separately from the ASD using autologous or bovine pericardıum. The mitral and tricuspid components were repaired. All pts with DORV were repaired with a transannular patch. Eight pts had no left AV valve regurgitation, two trivial and five mild. Ten pts had no right $A V$ valve regurgitation and five had mild right $A V$ valve regurgitation. Five of the 15 pts died postoperatively from different causes: pulmonary hypertensive crisis, low cardiac output syndrome or sepsis. No pt needed reoperation. There was no mortality in the follow-up period. The gradient across the RVOT ranged from $10-35 \mathrm{~mm} \mathrm{Hg}$. 


\section{P-25}

Doppler velocimetry of the aortic isthmus in human fetuses with abnormal velocity profiles in the umbilical artery

Sonesson $S-E$, Fouron J-C

St Justine Hospital, University of Montreal, Montreal, Canada and St Goran's Children's Hospital, Karolinska Institute, Stockholm, Sweden

Experimental studies, ${ }^{1}$ using the ultrasound Doppler technique, suggest that fetal blood flow redistribution during fetoplacental hemodynamic disturbances will be detected at an earlier and less severe stage in the aortic isthmus compared to the umbilical artery. This study was undertaken to investigate if findings supporting this concept could be demonstrated in the human fetus. In 100 pregnancies where the umbilical artery pulsatility index (PI) was found to be abnormal, it was demonstrated that qualitative changes, i.e. absence or reversal of enddiastolic flow, were related to the site of recording $(p<0.0001)$. These qualitative changes were more frequently found in the aortic isthmus (absent $28 \%$, reversed $41 \%$ ) compared to the fetal end of the umbilical artery (absent $20 \%$, reversed $19 \%$ ) ( $\mathrm{p}<0.0001$ ) and also more frequently found in the fetal end of the umbilical artery compared to the placental end (absent 13\%, reversed $2 \%)(\mathrm{p}<0.0001)$. No fetus was found where these changes were more severe in the umbilical artery compared to the aortic isthmus. Conclusively, this study on the human fetus confirm our previous observations of the aortic isthmus as a site for early detection of fetoplacental hemodynamic disturbances when using the ultrasound Doppler rechnique. Further studies are needed to establish the clinical value of these finding in terms of perinatal morbidity and, more importantly, of long-term neurological impairment.

1 Bonnun et al, Circulation 1993, 88216

P-26

Value of detailed, structured assessment and follow-up in children and adolescents with suspected Marfan syndrome

Wilson DG, Davies $S$, Stuart $A G$

Congenital Heart Disease Research Unit, University Hospital of Wales, UK

Marfan syndrome (MFS) is associated with increased risk of sudden death in young adults due to aortic root pathology. Early intervention with beta-blockers in patients with aortic root dilatation (ARD) may improve outcome in MFS. To provide detailed information about the progression of cardiovascular (CVS) findings in children and adolescents at risk of MFS and ARD, 73 patients ( $\mathrm{pts}$ ) with known/suspected MFS, or positive family history, referred for CVS assessment were studied. Pts were categorized as definite $(n=26,36 \%)$, probable $(n=8$, $11 \%)$, possible $(n=23,32 \%)$, or not $(n=16,22 \%)$ MFS, based on recognized clinical criteria. In the 34 pts with definite/probable MFS, CVS findings at referral were: abnormal clinical signs (murmur 4, click 9), ECG (partial right bundle branch block [PRB] 7, nodal beats 1, ventricular ectopics 1 , supraventricular tachycardia 1$)$, and echocardiogram $(A R D>97$ th percentile 26 , mitral valve prolapse [MVP] 8 , mitral regurgitation $[M R] 8$, aortic regurgitation $[A R] 6$, marked tricuspid regurgitation 1, abnormal septal motion 1). One pt was on beta-blockers at referral. Follow-up information (3-30 months) is available in 33/73 of pts referred (median number of visits 3 ). New CVS findings ar latest follow-up are: abnormal clinical signs (murmur 2, click 3 ), electrocardiogram (PRB 3), and echocardiogram (progression in ARD 13, new MR 5, new AR 4, abnormal septal motion 4, MVP 3). New information indicating definite MFS was obtained in 6 pts on follow-up. As a result of the information obtained, 7 pts have undergone MRI, and betablockers have been commenced in an additional 11. Detailed clinical assessment of young pts with an increased risk of MFS is valuable. Structured follow-up can provide new diagnostic information in pts where the diagnosis is not established. The early introduction of beta-blockers may improve the outcome in this group of at-risk patients.

\section{P-27}

Cardiomyopathy in the fetus-echocardiographic findings, outcome and associations

Simpson JM, Sharland GK

Department of Fetal Cardiology, Guy's Hospital London, United Kingdom

This study was a retrospective analysis of fetal cardiomyopathy (CMO) over a 13-year-period (1982-1995). CMO was defined as dilated if left and/or right ventricular dimensions were above the $95 \%$ confidence interval (CI) for gestarion with reduced function, and hypertrophic if ventricular wall thickness was above the $95 \% \mathrm{CI}$. The study aims were to quantify echocardiographic features of cardiomyopathy and to document associated disease states. Twenty-eight fetuses with CMO were identified which was dilated in 7 cases, hypertrophic in 13 and hypertrophic and dilated in 8 . Echocardiography: Dilated CMO: The right ventricle (RV) was affected in 4 cases, left in 1 and both in 2 . Left ventricular ejection fraction (LVEF) ranged from 5-22\% and RVEF from 5-39\%. Hypertrophic CMO: LVEF ranged from $44-78 \%$ and RVEF from 35-81\%. Hypertrophic and dilated CMO: The RV was involved in 2 cases, $L V$ in 2 and both in 4. LVEF ranged from $6-47 \%$ and RVEF from 9-38\%. Outcome and associations: Dilated CMO: There were 3 intrauterine deaths (IUD), one neonatal death (NND), one infant alive and one not known (NK). Associated diseases included fetal anaemia (2), maternal HIV infection (1), sialic acid storage disease (1) and endocardial fibroelastosis (EFE) (1). Hypertrophic CMO: There were 4 IUDs, 3 terminations, 2 NNDs, and 4 survivors. Associations included renal disease (5), diabetes (1), Noonan's syndrome (1), anaemia (1) and mitochondrial cytopathy (1). Hypertrophic and dilated: There were 3 IUDs, 3 NNDs, and 2 survivors. Associations included Coxsackie infection (1), mitochondrial cytopathy (1) and EFE $(\mathrm{n}=1)$. In conclusion, the causes of fetal CMO are diverse including renal, infective, genetic, and metabolic disorders. The prognosis of affected fetuses appears generally poor.

\section{P-28}

Rapid detection of chromosome $22 \mathrm{q} 11$ deletions by using microsatellite DNA markers

Bonnet D, Cormier V, Kachaner J, Szezepanski I, Souillard P, Std D , Munnich A, Lyonnet $S$

Service de Cardrologie Pédiatrique et INSERM U-393, Hôpital Necker/ Enfants Malades, Paris, France

Conotruncal cardiac malformations account for largely $50 \%$ of congenital heart defects in newborns. Recently, hemizygosity for chromosome $22 \mathrm{q} 11.2$ has been reported in patients with the DiGeorge/Velocardio-facial syndromes (DGS/VCFS) and phenorypically related disorders. We have explored the potential use of microsatellite DNA markers for rapid detection of 22q11 deletions in 20 newborns referred for conotruncal heart malformations with associated features of DGS/ VCFS. A failure of parental inheritance could be detected in $16 / 20$ $(80 \%)$ cases. The parental non-contribution was of maternal origin in $11 / 16$ and of paternal origin in 5/16. PCR-based genotyping using microsatellite DNA markers located (at loci D22S264, D22S941 and D22S944) within the commonly deleted region allowed to either confirm or reject a 22q11 microdeletion in 19/20 (95\%) of the cases within 24 hours. This test is now currently performed in the newborns referred to our institution for a conotruncal heart malformation as a first intention screening for 22q11 microdeletion in this population. Also, it will probably reduce the indication for fluorescent in situ hybridization studies to a limtted number of cases, as this procedure is particularly fast and cost efficient. It should be borne in mind, however, that a negative result does not rule out chromosome $22 \mathrm{q} 11$ deletions, as some patients may prove to have smaller deletions or point mutations within essential gene(s) mapping to chromosome 22q11. 


\section{P-29}

New development in pediatric cardiology-the miniature multiplane transesophageal transducer

Cromme-Dijkhus AH, Djoa KK, Bom N, Vermeulen A, Hess J

Pediatric Cardiology, Experimental Echocardiography, Sophia Children's Hospital, Erasmus University, Rotterdam, The Netherlands

Transesophageal echocardiography (TEE) has enriched the diagnostic capabilities of echocardiography in children. Recently, a pediatric multiplane TEE transducer became available. The tip dimensions of this transducer measures $27 \times 10.6 \times 7.9 \mathrm{~mm}$ and contains 48 transmitting elements. Two-dimensional imaging, $\mathrm{M}$-mode and all Doppler modalities are included. We evaluated 63 pes with this transducer. Ages ranged from 2 days to 16 years and weights from 3.6 to 67 kilograms. Eleven pts had complex heart disease, defined as the presence of at least 3 lesions; 17 pts had abnormal connection; and 34 pts only had 1 or 2 lesions. In $24 \mathrm{pts}$, prior surgery was performed. To assess the additional value of the multiplane, the visualization in the longitudinal planes of abnormalities were compared with those acquired in the initial transverse plane. Depending on the nature of the heart defect, 125 items were studied and judged. Validation was done by cardiac catheterization or surgery. Exclusively in the longitudinal planes $51(41 \%)$ items in 41 (65\%) pts were visualized. In the remaining 22 pts, valuable additional information was obtained about $74(59 \%)$ lesions, in the whole arc, where the longitudinal planes and the transverse plane are complementary to each other. Information obtained by TEE often influenced the decision making for surgery. Especially, in more complex heart defects or in patients with discordant connections, imaging in the longitudinal planes was far superior for the visualization of right and left ventricular outflow tract obstructions, semilunar valves abnormalities and baffle leakages. In conclusion, the multiplane pediatric transesophageal transducer has enriched the diagnostic possibilities in pediatric cardiology and gives more insight in the morphology of complex heart disease.

\section{P-30}

Left ventricular performance after mitral valve replacement in infants

Dinarevic $S$, Henein MY, Gibson DG, Shinebourne EA Royal Brompton Hospital, London, United Kingdom

All 6 infants undergoing mitral valve replacement since January 1990 survived. Age at operation was $11.2 \pm 4$ months with a mean weight of $6.6 \mathrm{~kg}$. Three had severe regurgitation, 2 mitral stenosis (MS) and 1 with a hypoplastic valve ring. Patients with complete or partial AVSD were excluded. $5 / 6$ had St. Jude's $(19 \mathrm{~mm}, 21 \mathrm{~mm} \mathrm{n}=3,23 \mathrm{~mm})$ and 1 Bjork Shiley prosthesis, $23 \mathrm{~mm}$. Papillary muscles were preserved in all. In those with MS, the prosthesis was placed in the left atrium proximal to the valve ring. Post-op LV function was assessed by echocardiographic $\mathrm{M}$-mode recording of long-axis function and transmitral Doppler and compared with 10 normals of similar age. At follow-up 17-45 months later, all were well. In systole, total LV long-axis excursion and timing were normal but peak shortening rate was reduced at the free wall $4,6 \pm 1.0$ vs $6.4 \pm 1.3 \mathrm{~cm} / \mathrm{s}, \mathrm{p}<0.01$. In diastole, peak lengthening rate was reduced at the free wall $8.0 \pm 3.2 \mathrm{vs} 12 \pm 2.4 \mathrm{~cm} / \mathrm{s}$ and the septum $6.3 \pm 1.4$ vs $9.0 \pm 3.0 \mathrm{~cm} / \mathrm{s}$, both $\mathrm{p}<0.05$, while the interval $A 2$ to peak lengthening $100 \pm 25$ vs $60 \pm 15 \mathrm{~ms}, \mathrm{p}<0.005$ was prolonged only at the free wall. Transmitral Doppler E-wave velocity was increased $110 \pm 14$ vs $80 \pm 12$ $\mathrm{cm} / \mathrm{s}$ as was $A$-wave $77 \pm 20$ vs $40 \pm 16 \mathrm{~cm} / \mathrm{s}$, both $\mathrm{p}<0.001$ and $E / A$ was reduced $1.5 \pm 0.5$ vs $2.2 \pm 0.5, p<0.05$. In conclusion, despite these timing and velocity differences, LV long-axis excursion and overall systolic and diastolic function were coordinate. Thus, mitral valve replacement can be successfully accomplished in infants. Preservation of papillary muscles is an important determinant of long-term coordinate LV function.

\section{P-31}

Heart rate behavior at different stages of congestive heart failure in children

Massin $M$, von Bernuth $G$

Department of Pediatric Cardiology, RWTH Aachen, Aachen, Germany

Heart rate variability (HRV) represents a noninvasive parameter for studying the autonomic control of the heart. The relation of measurements of HRV to these abnormalities in children with heart failure has not yet been examined. The present study examined HRV-indices determined from 24-hour Holter recordings in 130 children, aged 2 months to 12 yrs (mean 3 yrs), with congenital cardiac disease, the severity of heart failure being defined as NYHA functional class I to IV. Five time-domain measures (standard deviation (SD)) of all RR intervals over 24-hour (SDNN), mean of the SD (SDNNi) and SD of the means (SDANNi) of all RR in tervals for all 5-minute segments of the analysis, square root of the mean of the sum of squares of differences between adjacent RR intervals (rMSSD) and percentage of differences between adjacent RR intervals greater than $50 \mathrm{msec}$ for the whole analysis (pNN50)) and 4 frequency-domain measures $(\mathrm{Bl}<0.004 \mathrm{~Hz}$; B2 $0.004-0.04 \mathrm{~Hz}$; B3 0.04-0, $15 \mathrm{~Hz}$; B $40.15-0.4 \mathrm{~Hz}$ ) were compared to normal ranges established in 210 healthy normal children. A Z-score was established for each parameter according to the mean of all RR intervals over the length of the analysis and to the age of the patients. HRV is reduced in children with congenital heart disease, even in patients from NYHA class I. The overall 24-hour HRV and night/day heart rate ratio were reduced, de pending on the NYHA functional class. The most sensitive parameters were rMSSD, pNN50, B3 and B4. The balance B3/B4 is elevated in NYHA class I and class II but is reduced in class III and class IV with a virtual absence of any spectral component. It is concluded that HRV is reduced in children with congenital heart disease depending on the NYHA funcrional class. HRV-indices are sensitive markers of the clinical state.

P-32

Assessment of abnormal coronary arteries in patients with tetralogy of Fallot Norgaird G, Soukias ND, Carvalho JS, Shinebourne EA Royal Brompton Hospital, London, United Kingdom

Abnormal coronary arteries are reported in $2-10 \%$ of pts with tetralogy of Fallot (TOF), and its presence may alter time and type of repair. The purpose of the present study was to assess the accuracy of angiography and echocardiography in detecting abnormal coronary arteries in TOF $\mathrm{pts}$, and the impact of an abnormal coronary artery on surgical management. 102 pts were studied by aortography, 72 having a preoperative echocardiogram in which corona ry arteries were assessed. The aortogram was performed during routine preoperative cardiac carheterization in $\geq 45^{\circ}$ caudocranial and $20-30^{\circ}$, left anterior oblique view. The echocardiogram was performed from parasternal short-axis views. Abnormal coronary arteries were derected by angiography in 13 of 102 pts $(12.8 \%)$, and in 9 of 72 prs (12.5\%) who also had an echocardiogram performed. By angiography, all abnormal coronaries were detected, whereas one patient had a false-positive diagnosis (sensitivity $100 \%$, specificıty $98 \%$ ). Echocardiographic evaluation was inconclusive in 24 of 72 pts (33\%). In this subset of pts, 4 pts had abnormal coronary arteries, whereas the other 5 pts with abnormal coronary arteries were correctly diagnosed by echocardiography. Furthermore, one pt had a false-positive diagnosis of abnormal coronary artery. In $78 \%$ of the pts with abnormal coronary arteries, the surgical management was changed. In conclusion, aortography using the caudocranial view was accurate in detecting abnormal coronary arteries in TOF pts, whereas echocardiography was inconclusive in $33 \%$. We recommend that an aortogram using caudocranial view to be performed in all infants with inconclusive echocardiograms prior to surgery. 


\section{P-33}

VDD pacing in children-use of a single pass lead

Rosenthal E, Bostock J, Qureshi SA, Baker EJ, Tynan M

Department of Paediatric Cardiology, Guy's Hospital, London, United Kingdom

Concerns about preserving long-term venous access has restricted the use of DDD pacing in children. In order to overcome the potential complications of two lead systems, we have assessed the effectiveness of a single pass lead to provide VDD (synchronous) pacing in children with complete heart block (CHB). A single pass lead was implanted in 7 children aged 3.7 to 14.9 yrs (median $11.4 \mathrm{yrs}$ ), weighing 13.5 to $76 \mathrm{~kg}$ (median $38 \mathrm{~kg}$ ) with $\mathrm{CHB}$. Congenital $\mathrm{CHB}$ with a structurally normal heart was present in 5 . Two children developed $\mathrm{CHB}$ after surgical correction (dextracardia, TGA and a VSD in 1 and an AVSD and prosthetic left AV valve in 1). The older 4 children had 2-3 previous systems: 3 were converted from VVI/R systems after removal of a malfunctioning ventricular lead and 1 was converted from DDD after elective removal of an atrial J lead. A 58-cm single-pass lead (Vitatron 4, Medtronic 2, Intermedics 1) with a $13.5 \mathrm{~cm}$ spacing between the atrial sensing rings and lead tip was implanted via a subclavian vein puncture. The standard adult sized lead was chosen to permit coiling of redundant lead in the right atrium to avoid the need for lead advancement with growth of the child. In 1 child a complete loop was required to obtain adequate sensing. Satisfactory acute ventricular pacing thresholds and atrial sensing was obtained in all. Sapphire (4), Thera (2) and Unity (1) generators were attached to the lead and placed in a prepectoral pocket. Good atrial sensing and VDD pacing has been maintained with followup of 2-24 months (median 22 months). The 3 children whose systems were upgraded have increased their exercise tolerance. In conclusion, the benefits of physiological pacing can be obtained reliably in children with complete heart block by use of a standard single pass lead for VDD pacing.

\section{P-34}

A family with multiple cases of congenital cardiac malformations and conduction blocks

Sauer $U$, Berger $G$, Debrus $S$, de Meeus $A$, Bouvagnet $P$

Kinderklinik, Deusches Herzzentrum, München, Germany, Service de Pediatrie II, Hôpital St Charles, Montpellier, France

This large family is composed of 52 individuals in 5 generations with 9 cases of congenital heart defects (CHD) including double-outlet right ventricle ( 2 cases), tetralogy of Fallot ( 2 cases), patent ductus arteriosus, ventricular septal defect and aortic arch hypoplasia ( 2 cases), mitral valve prolapse ( 2 cases), and one case of a child who died of uncharacterized cyanotic CHD. In addition, a branch of this family demonstrated cardiac conduction blocks transmitted as an autosomal dominant trait. Five individuals are affected with a right bundle branch block (RBBB) (4) or a complete aurıculoventricular block (1). Two individuals with $\mathrm{RBBB}$ have in addition mitral valve prolapse. A striking observation in this family is that all CHD cases are in the 4 th and 5 th generations which suggests a mechanism of anticipation. Such a mechanism has been already described in 8 generic diseases, including fragile $X$ and Huntington syndromes. By contrast, the conduction block seems clearly autosomal dominant. Genoryping of this family in $19 \mathrm{q} 13$ and $22 \mathrm{q} 11$ chromosomal regions will be presented at the meeting. These regions have been implicated in familial conduction block and in conotruncal defects (CATCH22).
P-35

Psychosocial functioning of children, adolescents and young-adults operated upon for congenital heart disease-follow-up results of the "Rotterdam quality of life study"

Utens E, Verhulst F, Meijboom F, Hess J

Sophia Children's Hospital, Rotterdam, The Netherlands

The purpose of this descriptive and explorative study was to assess the long-term emotional, intellectual and social functioning after surgical correction for $\mathrm{CHD}$ in consecutive pts (712) who underwent their first open heart surgery between 1968 and 1980 in Rotterdam, and who were younger than 15 years at the time of surgery. At follow-up (1989-91), 498 pts participated in the psychological investigation (response rate, corrected for deceased pts and pts lost to follow-up: 87.7\%). Standardized assessment instruments were used. As to emotional functioning, parents of $144 \mathrm{CHD}$ children (10-15 yrs) completed the Child Behavior Checklist (CBCL) and 179 CHD adolescents (11-17yrs) completed the Youth Self-Report (YSR). On both instruments CHD children and adolescents obtained higher mean problem scores $(p<0.05)$ and scored more than twice as much in the psychopathological range $(\mathrm{p}<0.001)$ than same-aged peers from normative reference groups. A negative correlation was found between CBCL-total problem scores and IQscores of CHD children; for YSR-total problem scores of adolescents, no such relationship was found. The young-adult CHD pts (288; 18-35 yrs) reported significantly $(p<0.05)$ better emotional functioning (neuroticsm, self-esteem, hostility) than peers from normative reference groups and their results showed favorable outcomes on intellectual and social functioning (school/employment and leisure-time activities). No relationship was found between the cardiac diagnosis and the emotional functioning of children, adolescents and young-adults with CHD. In conclusion, in view of the results of the CHD children and adolescents in this study, we recommend that more attention should be paid to psychosocial counseling of CHD children and their parents.

\section{P-36}

Comparison of angioplasty and surgery for postoperative coarctation of the aorta

Veyre P, Bozio A, Jocteur-Monrozier D, Sassolas F, Revel D, Champsaur G, Ninet J

Hôpital Cardio-Vasculaire Louis Pradel, Lyon, France

The aim of this study was to compare the results of angioplasty $(A)$ and surgery (S) for postoperative coarctation of the aorta. Between 1976 to 1992,56 patients (pts) were treated by A ( $\mathrm{Gr} I=29 \mathrm{pts}$ ) or by $S$ ( $\mathrm{GrII}=27$ $\mathrm{pts})$. The study was retrospective without randomization.

$\begin{array}{lccc} & \text { Group I (29A) } & \text { Group II (27S) } & \text { p } \\ \text { Age at ini rual S (mean) } & 6 \text { months } & 2 \text { years } & <0.05 \\ \text { Range } & 2 \text { days-7.5 yrs } & 5 \text { days-15 yrs } & \\ \text { Age at postop A or S (mean) } & 6 \text { yrs } & 7 \text { yrs } & 0.28 \\ \text { Range } & 34 \text { days-27 yrs } & 35 \text { days-32 yrs } & \\ \text { Immediate results } & & & \\ \quad \text { Death } & 0 & 1 & \\ \text { Complicarions } & 2 & 9 & \\ \text { Mean SBP at rest (mm Hg) Before } & 134 & 133 & \mathrm{NS} \\ \text { Mean SBP (mm Hg) After } & 120 & 121 & \mathrm{NS} \\ \text { BP gradient (mm Hg) Before } & 36 & 45 & \\ \text { BP gradient (mm Hg) After } & 21 & 10 & <0.05 \\ \text { Doppler gradient (V2-V1) (mm Hg) B } & 52 & 51 & \mathrm{NS} \\ \text { Doppler gradient (V2-V1) (mm Hg) A } & 32 & 12 & <0.05 \\ \text { Mean hospital stay (days) } & 6 & 22 & <0.01 \\ \text { Long-term results } & & & \\ \text { Mean follow-up (years) } & 1.5 & 5.4 & <0.05 \\ \text { Doppler gradient (V2-V1) (mrn Hg) } & 33 & 15 & <0.05\end{array}$

The rate of complications and the short hospitalization stay without a thoracotomy obviously are in favor of angioplasty to treat restenosis of coarctation of the aorta. But as immediate- and long-term gradient reduction are concerned, the surgical treatment seems more efficient. Further prospective randomized study is mandatory and seems justified from a ethical point of view by these results in order to eliminate the methodologic error of such a retrospective study. 
P-37

Adenosine sensitive right ventricular tachycardia in childrenimpact of verapamil on QT dispersion

Çeliker A, Alehan D, Koçak G, Özme S

Pediatric Cardiology Unit, Hacettepe University, Ankara, Turkey

Right ventricular outflow tachycardia is a distinct subgroup of idiopathic ventricular tachycardia (VT) with characteristic clinical and electrophysiologic properties that have not been studied extensively in children. This study was designed to assess the efficacy of adenosine and verapamil in children with right ventricular tachycardia and to determine the effects of verapamil on QT dispersion in these pts. Eight pts ( 6 male, 2 female) with a mean age of $9.6 \pm 4.8$ years were enrolled in the study. Seven pts had VT and one pt had frequent ventricular ectopic beats originating from the right ventricle. Diagnostic tests including electrocardiogram (ECG), chest X-ray, echocardiogram, exercise testing, and 24-hour ambulatory ECG monitoring were performed in each pt. Durnng VT episodes intravenous adenosine was administered with increasing amounts $(100-300 \mu \mathrm{g} / \mathrm{kg})$ and was effective in terminating arrhythmia in all pts with a mean dose of $162 \pm 74 \mu \mathrm{g} / \mathrm{kg}$. Subsequently, verapamil was given orally, and during a mean follow-up period of $17 \pm 8$ months, favorable long-term results were obtained with an average dose of $6.2 \pm 2.2 \mathrm{mg} / \mathrm{kg} /$ day. QT dispersion decreased significantly from $81.2 \pm 8.3 \mathrm{msec}$ to $36.3 \pm 6.0 \mathrm{msec}(\mathrm{p}<0.001)$ in pts whose arrhythmia was suppressed by verapamil therapy. Adenosine can be effectively used in children to terminate VT originating from right ventricular outflow tract. Verapamil can be considered as the drug of choice for long-term therapy in these pts and QT dispersion is likely to be important in the assessment of the therapeutic efficacy of antiarrhythmic strategies.

\section{P-38}

Pulmonary balloon valvuloplasty for palliation of complex cyanotic congenital heart disease

Stimper O, Ptéchaud JF, Bonhoeffer P, Aggoun Y, Bonnet D, Sidt D, Kachaner J

Hôpital Necker-Enfants Malades, Paris, France

Pulmonary balloon valvuloplasty (PBV) was used in 18 parients (age 8 days-29 yrs; mean 5.4 yrs) with complex cyanotic congenital heart disease associated with pulmonary stenosis. This was performed in an attempt to improve cyanosis, thus allowing postponement of interim surgical palliation or complex repair. Seven pts had univentricular heart, 5 double discordance and 6 complex forms of transposition or double outlet right ventricle. Two had situs inversus, 1 right atrial isomerism and 4 dextrocardia. In 11 pts, PBV was performed as a primary procedure $(61 \%)$ and in 7 after previous palliation (39\%). Oxygen saturation $\left(\mathrm{SO}_{2}\right.$ ) prior to PBV ranged from $57-83 \%$ (mean $68 \%$ ). Balloon-to-annulus ratio measured 0.9-1.3 (mean 1.0). After PBV, $\mathrm{SO}_{2}$ increased by $1-31 \%$ (mean $15 \%$ ) to $75-91 \%$ (mean $84 \%$ ) $[\mathrm{p}<0.05]^{2}$. Mean pulmonary artery pressure increased by $0-14 \mathrm{~mm} \mathrm{Hg}$ (mean 4.3 $\mathrm{mm} \mathrm{Hg}$ ). Transient complete AV block was encountered in $1 \mathrm{pt}$. In 5 pts $(28 \%)$, there was no persistent improvement in cyanosis. In 4 this was due to increasing infundibular stenosis and in 1 due to poor streaming. All 5 underwent surgery within 4 weeks after PBV. In 13 pts (72\%), PBV was successful. Definitive surgery could be delayed by a mean of 1.2 years in $4 \mathrm{pts}$, and in $9 \mathrm{pts}(50 \%) \mathrm{PBV}$ was the final palliative procedure. Follow-up in this latter group is 2 wks- 5 yrs (mean 1 yr). $\mathrm{SO}_{2}$ range from $76-89 \%$ (mean $83 \%$ ) compared to $62-81 \%$ (mean $70 \%$ ) prior to PBV $[\mathrm{p}<0.05]$. In conclusion, $\mathrm{PBV}$ is a safe and effective technique in the palliation of pts with complex cyanotic congenital heart disease associated with pulmonary stenosis. It allows postponement of complex surgical repair or the creation of a venous rather than an arterial shunt in young infants and children.
P-39

Clinical and histopathological features of myocardial infarction in perinatal period

Kokai GK, Jovanovic I, Ljubuc A, Parezanovic V, Simenunovic S,

Ceranic L, Doukıc M

Institute of Gynecology \& Obstetrics and University Children's Hospital, Belgrade, Yugoslavia

The aim of this study is to present our experience with myocardial infarction (MI) in fetuses with no coronary artery abnormalities in twelve fetuses and preterm babies having myocardial necrosis and/or calcification proven by autopsy. Five of those had congenital heart disease (CHD) diagnosed antenatally (group I). Seven others suffered from perinatal asphyxia unrelated to $\mathrm{CHD}$ (group II). An echo was done in all fetuses from group I, and standard intensive care monitoring in group II. All hearts were measured, photographed, examined macroscopically and histologically. In group I, 4 feruses had severe obstructive $\mathrm{CHD} ; 1$ was a parasitic twin of pregnancy with total reverse arterial perfusion. Feral echo showed hyperechogenic areas in myocardium in all fetuses, and a huge left ventricle aneurysm in one of them. MI zones were found distally from the obstructive lesion, predominantly in subendocardial regions and papillary muscles, except in one with transmural extension (aneurysmatic wall). Macroscopic calcification was noticeable in three hearts. In group II, all babies were preterm suffering from severe perinatal asphyxia. Of these, 3 had pathologic findings (transudate in serosal cavities) suggesting possible infection, but detailed virological investigations were not performed. Macroscopic evidence of MI was present in two cases with intraventricular mural thrombus in one. MI zones were localized in the inner half of the left ventricle myocardium. Florid, acure, coagulative necrosis of myocytes with focal hemorrhage, mild-moderate inflammatory cell infiltrate without calcification were evident on histology. Striking difference in histology of MI between groups could be explained by diverse nature and duration of underlying pathology. Early clinical diagnosis of $\mathrm{MI}$ is possible by fetal echo and ECG postnatally.

\section{P-40}

Circadian distribution of premature ventricular contractions-a useful prognostic parameter in the evaluation of ventricular arrhythmias in children

Simeunovic S, Vukomanovic G, Gajic Z, Jovanovic I, Ceranic LJ, Djukuc M, Milincic Z, Parezanovic V, Pavlovic SU, Milasinovic G, Zivkovic M University Children's Hospital, Belgrade, Yugoslavia

Ninety-oneptsunderwent a protocol including echo, ECG, Holter monitoring, stress test and late potentials on signal-averaged ECG. Two groups were formed: A (71/91 pts) with normal echo finding and B (20/91 pts) with concomitant heart disease (congenital heart disease excluded). In group A, based on standard prognostic parameters and follow-up, pts were divided in two subgroups: A1 (41/71 pts)-asymptomatic pts with favorable prognostic parameters, without deterioration of arrhythmia during follow-up; and subgroup A2 (30/71 pts)-symptomatic pts, pts with "high grade" PVC: multiform PVC, couplets, nonsustained VT, frequent PVCs (over 10,000/ 24 hrs nor suppressed by exercise). We sorted out pts with PVC increasing in number during night sleep (positive night distribution-PND). In group $A$, $32 / 71$ pts displayed $P N D$, and $14 / 20$ pts in group $B(p<0.05$ ). In subgroup $A 1$, there were $5 / 41$ pts with $P N D$ and $27 / 30$ pts in subgroup $A 2(p<0.01)$. In subgroup A2, 11/30 pts had favorable prognostic parameters at first examination burduring follow-updeveloped arleast 2 unfavorableprognostic parameters; 10/11 of these pts had PND at first examination. In group B, all pts who had paired PVC (11) and nonsustained VT (3), also had PND. In both groups, pts with PND had worse response to beta blocking treatmentin 5/12 pts in group A, beta-blockers failed and all five pts displayed PND, while $7 / 12$ pts responded to treatment and only one had PND ( $p<0.01)$; in $8 / 14$ pts in group $B$, beta-blockers failed and 7 of these pts had PND, while of 6/14 who were successfully treated, only one displayed PND $(p<0.05)$. We conclude that positive night distribution of PVCs is a simple and useful prognostic parameter in the evaluation of children with premature ventricular contractions. 
P-41

Morphologic changes of the fetal heart

Wenink ACG, Knaapen MWM

Department of Anatomy, University of Leiden, Leiden, The Netherlands

Fetal cardiology and physiologic experiments on embryonic and fetal hearts need detailed information on the changes of fetal cardiac morphology. To establish these changes, fetal rats from 11 to 17 embryonic days, i.e. from shortly after looping of the heart tube to an advanced fetal stage, were studied by light as well as electron microscopy. The changes were quantified by stereologic methods. Initially, the left ventricle is the main contractile compartment. The myocardial volume of the right ventricle is increasing secondarily to reach similar values only around the time of septation. The atrioventricular canal and the outflow tract are proportionally very important components of the heart at 11 days, but their proportional volumes decrease considerably. This volume decrease is accounted for by a relative stop of mitotic activity. In the atrioventricular canal the myocytes contain $30 \%$ of glycogen throughout development. Glycogen storage does not show the features of immediate metabolic availability, but could rather function as a physical stabilizer of the canal. By adding to its rigidity it supports the closing mechanism by the endocardial cushions as long as valves have not yet developed. In the sinuatrial junction, which attains a valve-like function when cardiac pressures increase, the cellular glycogen fraction increases from less than $5 \%$ at 11 days to $30 \%$ at 17 days. Myofibril assembly is still poor in these stages. The volume fraction in the left ventricle rises from $15 \%$ at 11 days to only $25 \%$ at 17 days. Moreover, the degree of orientation of myofibrils is generally poor. An exception is formed by the outflow tract where the myofibrils show clear alignment. Here, a relatively advanced contractile function would serve to prevent regurgitation in the absence of arterial valves. The above data show that the function of the embryonic heart must differ considerably from that of the mature organ. The latter is only gradually established, but not completed before birth.

\section{P-42}

Anticoagulation defects following Fontan

Jahangiri M, Shore D, Kakkar V, Lincoln C, Shinebourne ES

The Royal Brompton Hospital, London, United Kingdom

Recently we reported a high incidence of thromboembolism in pts who underwent the Fontan procedure and its modifications. While hemodynamic factors may well contribute to this, recent evidence suggests that coagulation factor abnormalities may also play a role. We therefore set out to investigate the coagulation status in a group of pts who had undergone the Fontan procedure. The study population consists of 20 children who had undergone the Fontan procedure and its modifications. They were examined for coagulation factor abnormalities and their serum albumin, total protein and liver enzymes were measured. The median age at the time of surgery was 6.2 years with a male-tofemale ratio of $2.3: 1$, the median time from Fontan repair was 4.9 years (range 18-76 months). Protein $C(p<0.001)$, protein $S(p<0.005)$ and factor VII $(p<0.001)$ were significantly lower than the normal range. The changes in serum albumin and total protein, factors II, VII, LX and $\mathrm{X}$ were not significant. It is possible that a deficiency in protein $\mathrm{C}$, protein $S$ and factor VI partly account for the high incidence of thromboembolism following Fontan type repair. The risk of long-term anticoagulation should be weighed against the best palliative procedure for these pts. We suggest that reduced protein $C$, protein $S$ and factor VII in this group of pts should be regarded as risk factors and that such pts should be anticoagulated.
P-43

Bipolar epicardial steroid-eluting pacing leads for atrial and ventricular pacing in children

Witsenburg $M$, Kruit MN, van Vliet AM, Bogers AJJC

Departments of Pediatric Cardiology, Sophia Children's Hospital and Cardropulmonary Surgery, Dijkzigt University Hospital, Rotterdam, The Netherlands

Epicardial pacing has been reported to be of limited value because of threshold rise at short term, especially in infants and children. The use of steroid-eluting leads may prevent threshold rise. Between August 1990 and April 1995, a consecutive series of 23 pts (pts), 8 girls and 15 boys, underwent implantation of an epicardial pacemaker system. Indication for pacing were non-surgical AV-block in 14 pts, surgical $\mathrm{AV}$-block in 6 and sinus node dysfunction in 3. 34 bipolar Medtronic steroid-eluting pacemaker leads were implanted, 23 on the ventricle and 11 on the atrium. Ventricular leads used were SP2140 in 4, SP2156 in 1 and 10366 in 18. Acrial leads were SP2140 in 1, SP2156 in 1 and 10366 in 9 pts. Mean follow-up was 28 (5-60) months. Three pts died of non-pacemaker or lead related events (pulmonary hypertension in 1 and ventricular dysfunction in 2). Only 2 leads of the early type (SP2140) had to be replaced within 2 yrs, 1 due to high threshold and 1 due to inadequate fixation. All other leads function well during followup. Threshold levels (mean $\pm \mathrm{SD}$; msec at $2.5 \mathrm{~V}$ ) were low at implantation and no significant changes were observed during follow-up.

$\begin{array}{lcccc}\text { Lead position } & \text { Implant } & 3 \text { months } & 12 \text { months } & 24 \text { months } \\ \text { Ventricle } & 0.21(0.15) & 0.29(0.26) & 0.26(0.25) & 0.22(0.11) \\ \text { Atrumm } & 020(0.20) & 0.11(0.07) & 0.13(0.13) & 017(016)\end{array}$

In conclusion, bipolar epicardial steroid-eluting pacing leads in infants and children show good performance at mid-term follow-up, with persistence of low threshold levels. Threshold levels compare well with endocardial pacing. Epicardial pacing with bipolar steroid-eluting leads is the preferred alternative in infants and young children.

\section{P.44}

Truncus arteriosus communis-indication for operation and prognosis after primary correction

Eicken A, Schumacher G, Meisner H, Balling G, Lorenz HP, Häbnel JC, Bühlmeyer $K$

Deutsches Herzzentrum, München, Deutschland

From 1974 to October 1995, 97 children with TAC (TAC A1 or A2 $n=85$, TAC A $3 n=5$, TAC A $4 n=7$ van Praagh class) underwent primary surgical correction. In all cases a homograft or xenograft conduit was used as a pulmonary valve. 54/78 (69\%) pts with TAC A1/A2 survived, median age at operation in this group was 4.1 (range 0.5-19) months. Seven children with TAC A1/A2 were corrected after the age of two years (median age 5.9 years) and of these pts 4 survived (57\%). $3 / 5$ (60\%) infants with TAC A3 and 3/7 (43\%) infants with TAC A4 survived corrective surgery (median age at operation 1.6 and 1.2 months respectively). Retrospective data analysis was performed in these $97 \mathrm{pts}$ to assess risk factors for primary correction in TAC. Number of truncus valve leaflets, TAC type $\mathrm{A1}$ or $\mathrm{A2}$, grade of malalignment and size of VSD, central pulmonary branch stenosis, pulmonary vascular resistance (if corrected early) and arterial $\mathrm{SO}_{2}$ had no influence on postoperative outcome. However, a TAC A3 or A4, truncus valve regurgitation $>2$ grade (even more in combination with significant valvar stenosis), coronary abnormalities, preoperatıve mechanical ventilation of more than 1 week and an associated DiGeorge syndrome were significantly associated with a poorer outcome. In conclusion, primary surgical correction of pts with TAC A1 and A2 without any of the above mentioned risk factors can be performed with a $85 \%$ chance of longterm survival. The best age for operation is between 6 and 12 months. 


\section{P-45}

Determinants of exercise capacity and exercise arterial oxygenation after atrial redirection for transposition of the great arteries Gilljam T, Eriksson BO, Sixt R

Pediatruc Cardiology and Pediatric Clinucal Physiology, Ostra Sjukbuset, Goteborg, Sweden

To assess the interplay between pulmonary gas exchange and cardiac output after atrial redirection for transposition, 17 Mustard and Senning pts had a maximum ergometer test with simultaneous analyses of expiratory gases, arterial blood gases and cardiac output. Two had moderate pulmonary hypertension, 7 had systemic ventricular dysfunction at rest, 7 had a tiny baffle defect and 1 had a small atrial shunt. Another patient with a considerable atrial shunt and 1 with tricuspid regurgitation were excluded from some analyses. Maximum $\mathrm{O}_{2}$ uptake per weight $(29.6 \mathrm{ml} / \mathrm{kg} / \mathrm{min})$ and heart rate $(167 \mathrm{bpm})$ were low $(\mathrm{p}<0.001)$. During exercise, small right-to-left shunts were detected in 5 pts. The physiologic dead space increased abnormally in both subjects with $\mathrm{PHT}$ and in one other subject. Arterial $\mathrm{O}_{2}$ partial pressure and $\mathrm{SO}$, fell in all subjects during exercise $(p<0.0001) .15 / 16(94 \%)$ had a high alveolar-arterial $\mathrm{O}_{2}$ partial pressure difference during exercise. Cardiac output was low $(\mathrm{p}<0.0001)$, but below 2 SD in only $4 / 15(27 \%)$. Actually, in $6 / 12$, more than $90 \%$ of the available oxygen was consumed at peak exercise, as calculated from arterial oxygen content and arteriovenous $\mathrm{O}_{2}$ difference. $13 / 15$ had falling stroke volumes during exercise. The subjects with systemic ventricular dysfunction at rest were not among those with the poorest stroke volume response. The low exercise capacity was caused by a combination of chronotropic incompetence and falling stroke volumes. The abnormal stroke volume response may be caused by slow ventricular filling at higher heart rates due to poor atrial function, which may also be a contributing factor to the low maximum heart rate. The impaired arterial oxygenation during exercise may be caused by a combination of pulmonary ventilation/perfusion mismatch, a low mixed venous content and some atrial shunting in some.

\section{P-46}

State of differentiation of smooth muscle cells in intimal proliferation of human aortic coarctation.

Jiménez $M$, Daret D, Laborde N, Roques X, Choussat A, Baudet $E$, Bonnet J

Hôpital Cardiologique, CHU de Bordeaux, Pessac, France

The nature and mechanisms of aortic narrowing in human coarctation are poorly understood. In order to characterize the component of intimal thickening of coarctation, narrowed segments of aorta, obtained after surgery from 10 children (mean age 15.8 months, range 15 days4 years) were analyzed by immunocytochemical labeling. Hematoxlineosin staining demonstrated a permanent dramatic increase in intimal thickness. The cellular component analysis of intimal thickening showed only smooth muscle actin positive cells oriented in an opposite way than the medial cells. No cells were stained by monoclonal antibody against macrophages ( $\mathrm{Ham} 56$ ) or lymphocytes (Pan T). Masson's trichrome and Weigert staining displayed a strong increase in collagen in the intima. In order to characterize the differentiation state of smooth muscle cells (SMC) in intimal thickening, SMC markers were studied by specific monoclonal antibodies. The important decrease of expression of smooth muscle-myosin heavy chain, heavy caldesmone, metavinculine and desmin suggested a strong differentiated state of SMC reminding condition of intimal SMC of ductus arteriosus (DA). No nuclei expressed proliferating cell nuclear antigen (PCNA). These data indicate that human aortic coarctation is characterized by intimal recruitment of differentiated non-proliferating SMC, identical to the well-defined SMC of the ductus arteriosus and suggesting the reality of ductal migrating SMC hypothesis to explain human aortic coarctation. The migration factors of ductal SMC are to be determined.
P-47

Severity of mitral regurgitation assessed by intraoperative pulmonary venous flow analysis

Kececioglu D, Gehrmann J, Kehl HG, Vielhaber H, Scheld HH, Vogt J Westfälische Wilhelms Universität, Minster, Germany

Severe mitral regurgitation (MR) after repair of an atrioventricular septa defect may result in significant morbidity and mortality. The aim of this study was to analyze the value of pulmonary venous flow in the intraoperative assessment of MR. A total of 43 intraoperative transesophageal echocardiographic studies were performed in 33 children (age 0.02-16.6 yrs) undergoing repair of an atrioventricular septal defect. The severity of MR was assessed by color flow mapping (CFM), relating MR jet area to left atrial area (MR\%). Pulmonary venous Doppler tracings (PVD) were analyzed for peak systolic (SV), peak systolic reversed (SRV) and peak diastolic velocities (DV) and for their corresponding velocity time integrals (SI, DI, SRI). The time interval of systolic reversed flow was related to the QT interval (SR/QT). SV/SRV and SI/SRI moderately correlated with MR\% $(r=0.61$ resp. $r=0.66)$. Systolic reversed flow was detected in 8 of 9 pts with MR\% $>30$, but was also observed in the presence of less severe MR in pts dependent on cardiac pacing. SR/QT was higher in pts in sinus rhythm than in those with cardiac pacing $(0.6$ versus 0.3$)$. When pts with cardiac pacing were excluded, a betrer correlation ( $r=0.77)$ was found between SV/SRV and MR\%. Sensitivity and specificity values reached $100 \%$ when $M R \%>30$ and systolic reversed flow were combined to select pts requiring urgent surgery for MR. In conclusion, systolic reversed pulmonary venous flow is a strong indicator for severe MR. CFM and PVD should be used complementary to assess the significance of any residual MR after repair of an atrioventricular septal defect.

\section{P-48}

Cor triatriatum dexter in infancy-symptoms, imaging techniques and course

Mühler EG, Franke A, Hausler M, Messmer BJ, von Bernuth $G$ Departments of Pediatric Cardiology, Cardiovascular Surgery and Internal Medicine I, Aachen, Germany

Cor triatriatum dexter (CTD) is a very rare malformation, with most cases being recorded at necropsy. Four cases of CTD diagnosed during infancy are described. Recurrent cyanosis was the leading symptom in all. Clinical examination, ECG and chest radiograph showed no abnormalities; transthoracic echocardiography at the first investigation missed the diagnosis of CTD in all pts. Right-to-left shunt at the atrial level, proven by contrast echocardiography $(n=2)$, was explained by mild Ebstein's anomaly or persistent pulmonary hypertension of the newborn. A thin right atrial membrane close to the tricuspid valve with slightly accelerated diastolic flow $(\max 1.6 \mathrm{~m} / \mathrm{sec}$ ) was detected at repeated transthoracic echocardiography in three infants. In the fourth case, the only one with enlargement of the caval veins as a diagnostic hallmark, the highly obstructive membrane was clearly visualized only by means of transesophageal echocardiography. Spontaneous normalization of arterial oxygen saturation occurred in two pts until the third month of life. Cardiac catheterization was performed in the remaining two: a non-obstructive right atrial membrane combined with a $20 \%$ right-to-left shunt preferentially from the inferior caval vein to the left atrium was proven in a 5-month-old boy; in the last patient, the membrane caused a mean pressure gradient of $9 \mathrm{~mm} \mathrm{Hg}$ and was surgically excised. In conclusion, CTD has to be excluded in neonates with cyanosis and atrial right-to-left shunt. The course of CTD varies from "spontaneous healing" (mild obstruction, spontaneous closure of the fossa ovalis defect) up to progressive obstruction with indication to surgery. 


\section{P-49}

Treatment of supraventricular tachycardias with radiofrequency catheter ablation in children

Walfridsson H, Eriksson B, Gilljam T, Edvardsson $N$ Sahlgrens University Hospital and Östra Sjukhuset, Göteborg, Sweden

Paroxysmal supraventricular tachycardia (PSVT) is common in children. Catheter ablation with radiofrequency energy (RF) is well established in adults when treatment with antiarrhythmic drugs is insufficient. We report our experience with RF in 42 children. From 1991 to 1995, we performed 510 RF; 42 were in children less than 18 years old. Nineteen had overt WPW syndrome, 14 had concealed WPW syndrome, 2 had another preexcitation syndrome (Mahaim) and 7 pts had AV-nodal reentrant tachycardia (AVNRT). Mean age was 13.4 (range 4-17) years. Nineteen pts had overt WPW; one pt had a concealed accessory pathway that was ablated in the same procedure. The first RF was successful in 16, one had a late cure, one was ablated in a second procedure and one is waiting for a second procedure. In only one was it not possible to treat with RF. Fourteen pts with concealed WPW were treated successfully in the first procedure without recurrence. Seven pts with AVNRT were treated successfully in the first procedure. One pt had a recurrence that was treated with a second RF. The first two pts were ablated against the "fast pathway," while the others were treated against the "slow pathway." Two pts had Mahaim fibers. One was treated against the fast pathway, and the second failed the first attempt and is waiting for a second RF. In conclusion, RF could be performed with adequate safery and with equally good results as in adults in our study population ranging from 4 to 17 years of age.

P-50

Surgical versus interventional closure of atrial septal defect-the acute adaptation of the right ventricle

Berger F, Jin Z, Ichıhashi K, Alexi-Meskishvili $V$, Lange PE German Heart Centre Berlin, Berlin, Germany

To compare the acute hemodynamic response of the right ventricle (RV) to surgical (OP) or interventional (INT) closure of an atrial septal defect (ASD), $R V$ size and function were analyzed by echo in 47 pts matched by age, body surfacearea (BSA) and size of theleft-to-rightshunt (Qp:Qs). Those, in whom the relation of defect size to septum length or the localization of the ASD precluded an INT, underwent OP.

$\begin{array}{lcc} & \text { OP group }(\mathrm{n}=23) & \text { INT group }(\mathrm{n}=24) \\ \text { Age (years) } & \text { Medan } 105 \text { (range 0 8-56) } & \text { Median } 83(\text { range 25-48) } \\ \text { BSA (m) } & 1.14 \pm 057 & 1.12 \pm 04 \\ \text { Qp Qs } & 1.87 \pm 028 & 169 \pm 036\end{array}$

Mean values and SD of end-diastolic volume(RVEDV); end-systolic volume (RVESV); stroke volume (RVSV), ejection fraction (RVEF) were determined and compared to normal. Results are included in the Table below.

$\begin{array}{lcc} & \text { Before OP }(\mathrm{n}=23) & \text { Before INT }(\mathrm{n}=24) \\ \text { RVEDV }\left(\mathrm{m} / / \mathrm{m}^{2}\right) & * 41.56 \pm 11.72 & * 31.44 \pm 8.46 \\ \text { RVESV }\left(\mathrm{ml} / \mathrm{m}^{2}\right) & * 1666 \pm 6.7 & * 1311 \pm 460 \\ \text { RVSV }\left(\mathrm{m} / \mathrm{m}^{2}\right) & * 24.9 \pm 7.87 & * 1868 \pm 571 \\ \text { RVEF }(\%) & 60 \pm 10 & 59 \pm 8 \\ & \text { After OP }(\mathrm{n}=23) & \text { After INT }(\mathrm{n}=24) \\ \text { RVEDV }\left(\mathrm{m} / \mathrm{m}^{2}\right) & 22.78 \pm 10.33 & 2328 \pm 659 \\ \text { RVESV }\left(\mathrm{ml} / \mathrm{m}^{2}\right) & * 10.90 \pm 4.96 & * 1049 \pm 317 \\ \text { RVSV }\left(\mathrm{m} / \mathrm{m}^{2}\right) & 11.88 \pm 5.82 & 1279 \pm 402 \\ \text { RVEF }(\%) & * 52 \pm 7 & * 55 \pm 5\end{array}$

RV volumes in pts with ASD (OP- and INT-group) were significantly enlarged $(\mathrm{p}<0.05)$. RV volumes and RVEF decreased significantly $(\mathrm{p}<0.05)$ after closure, and RVEDV and RVSV even normalized within 3 days after closure $(p<0.05)$. The acute adaptation of RV after closure of an ASD seems tobeindependent of closure technique. The RV sizenormalizes, when Qp:Qs is $<2$. The impaired RV function could be due to altered loading conditions.
P-51

Cardiac dysrhythmias in Ebstein's anomaly after surgical treatment Ewert P, Nagdyman N, Ublemann F, Hetzer R, Lange PE German Heart Institute Berlin, Berlin, Germany

Ebstein's anomaly is most commonly associated with a variety of cardiac dysrhythmias. This study evaluated how surgical treatment influences preoperative existing cardiac dysrhythmias. The study group consisted of 28 pts ( 13 female, 15 male) age one day to 65 years (mean 26 years) undergoing surgical treatment from October 1988 to September 1995. Closure of atrial septal defects was performed in $90 \%$, tricuspid valve reconstruction in $72 \%$, and $18 \%$ received a prosthetic valve. The remaining pts underwent surgical treatment for other malformations. All pts had multiple ECGs and Holters before and after the operation. In the preoperative period, cardiac dysrhythmias consisted of the following: premature beats in three pts, supraventricular tachycardia in two cases, atrial flutter in one, atrial fibrillation in four, Wolff-ParkinsonWhite (WPW) syndrome in two, AV conduction disturbance grade $\mathrm{I}$ in five, $A V$-node rhythm in one, incomplete and complete right bundle branch block in 20 and in 8 pts, respectively. After surgical therapy, premature beats were still present in 2 pts. Atrial flutter and atrial fibrillation occurred in only two pts. WPW-syndrome remained in one case but was successfully treated by accessory pathway ablation. AVconduction disturbance grade I was observed in three pts, grade II was new in one case after operation. AV-node rhythm changed in one case to sinus rhythm, but in another pt sinus rhythm turned to AV-node rhythm. One case of sinus bradycardia was noted. Fifteen pts had complete right bundle branch block. Pacemaker implantation was necessary in five pts with new the onset of complete AV-node block. Two pts had sinus thythm in contrast to preoperative rhythm. In conclusion, surgical treatment of pts with Ebstein's anomaly does not improve preexisting cardiac dysrhythmias.

P-52

Tissue plasminogen activator versus streptokinase for treatment of thrombosis and thromboembolism in infants and children

Löning L, Paul T, Bergmann F, Luhmer I, Kallfelz HC Pediatric Cardiology, Hannover Medical School, Hannover, Germany

Between June 1985 and July 1995 thrombolytic therapy was performed in 34 children (mean age 3.2 years, mean body weight $14 \mathrm{~kg} ; 27 / 34$ after cardiaccatheterization) with venous or arterial thrombosis. In 19/34 pts streptokinase was given (initially $3000 \mathrm{U} / \mathrm{kg}$ over $30 \mathrm{~min}$, subsequently $1000 \mathrm{U} / \mathrm{kg} \cdot \mathrm{h}$ ) for 1 to 82 (mean 20.2, median 16) hours. 16/34 pts were treated with tissue plasminogen activator ( $\mathrm{tPA}$; initially $0.12 \mathrm{mg} / \mathrm{kg}$ over $10 \mathrm{~min}$, followed by 0.1 to 0.4 [max 15 ] $\mathrm{mg} / \mathrm{kg} \cdot \mathrm{h}$, additional heparin $100-400 \mathrm{U} / \mathrm{kg} \cdot \mathrm{d}$ ) over 0.5 to 72 (mean 14 , median 4.2 ) hours. One child was treated with $\mathrm{t}$-PA after unsuccessful therapy with streptokinase.

\begin{tabular}{lrlrl} 
& Streptokınase $(\mathrm{n}=19)$ & \multicolumn{2}{c}{$\mathrm{t}-\mathrm{PA}(\mathrm{n}=16)$} \\
Success & $13 / 19$ & $(74 \%)$ & $13 / 16$ & $(81 \%)$ \\
Rash, fever & $2 / 19$ & $(10 \%)$ & - & \\
& Subsequent urokinase & & \\
Bleeding (total) & $6 / 19$ & $(32 \%)$ & $10 / 16$ & $(63 \%)$ \\
$\quad$ Mucosal & - & $2 / 10$ & \\
Puncture site & $6 / 6$ & $8 / 10$ & \\
$\quad$ Requiring transfusion & $3 / 6$ & $2 / 10$ &
\end{tabular}

The success rate was not different in pis treated with streptokinase or $t$-PA. Duration of treatment was significantly shorter in the t-PA group. Allergic reactions were only observed in children treated with streprokinase. Significant more bleeding complications had to be treated in the t-PA group. 


\section{P-53}

Growth of the tricuspid valve after laser or radiofrequency pulmonary valvotomy in neonates with pulmonary atresia and intact ventricular septum

Ovaert C, Rosenthal E, Qureshi SA, Baker EJ, Tynan M

Guy's Hospital, London, United Kingdom

Transcatheter valvotomy for pulmonary atresia with intact ventricular septum (PA-IVS) is a recent development. Little is known about the consequent growth of the right ventricle. Five patients (pts) with PAIVS have been followed for a mean of 52 (range 37-63) months after transcatheter pulmonary valvotomy (laser in 3 , radiofrequency in 2). Mean age at valvotomy was 6.2 (range 1-9) days, mean weight 2.7 (range $2.3-3.1) \mathrm{kg}$. Additional procedures included balloon dilatation of the arterial duct in 2 pts on 3 occasions, stenting of the duct in 1 and balloon dilatation of the pulmonary valve (PV) in 4. An atrial septal defect was closed surgically in 1 pt. The tricuspid (TV) and mitral valve (MV) annulus were measured on echocardiography in an apical four-chamber view and relared to body surface area. Results are expressed as means for the group.

$\begin{array}{lcc} & \text { Before valvotomy } & \text { Latest measurements } \\ \text { TV }(\mathrm{mm}) & 9.8 & 206 \\ \text { Z-score TV } & -0.68 & -186 \\ \text { MV }(\mathrm{mm}) & 155 & 23.4 \\ \text { Z-score MV } & +106 & -1.06 \\ \text { TV/MV ratıo } & 063 & 0.88\end{array}$

TV dimensions were at the lower end of the normal range before the procedure and increased steadily. The TV Z-scores however slightly decreased in all and fell below -2 for 3 pts. MVZ-scores remained within normal limits in all pts. TV/MV ratio increased slightly in all but only reached 1 in 1 pt. Current PV gradients are 4 to $49 \mathrm{~mm} \mathrm{Hg}$ (mean 25.7 $\mathrm{mm} \mathrm{Hg}$ ). Transcatheter pulmonary valvotomy in neonates with PAIVS leads to satisfactory RV function from a clinical point of view, although TV annulus growth rate remains suboptimal.

\section{P-54}

Troponin- $T$ as a diagnostic tool in ischemic myocardial dysfunction of the newborn

Agnoletti G, Panteghini M, Borghi A

Divisione di Cardiologia, Ospedali Runniti, Bergamo and Laboratorio Analisi, Spedali Civili, Brescia, Italy

IMDN is a pathological condition whose prognosis can be severe; its diagnosis is not supported by the measurement of creatinine kinase $(\mathrm{CK})$ and $\mathrm{CK} M B$ in the serum, due to the profound changes occurring in the CK system during the perinatal period. Our aim was to investigate the possibility of using TnT-release as a marker of IMDN. For this purpose we measured CK-, CK MB- and TnT-release, on the second and fourth day of life, in 52 newborns (age 28 to 40 gestational weeks), 44 without and 8 with IMDN. CK- and CK MB-release was positively related to gestational age, while TnT-release was not. Infants with IMND had a CK- and CK MB-release ( $450.7 \pm 59.3 \mathrm{UI} / \mathrm{l}$ and $48.6 \pm 21.2$ $\mu \mathrm{g} / \mathrm{l})$ similar to that of infants without IMND $(630.7 \pm 74.1 \mathrm{UI} / \mathrm{l}$ and $43.8 \pm 12.5 \mu \mathrm{g} / \mathrm{l})$. On the contrary, TnT-release was significantly higher in newborns with IMDN $(0.70 \pm 0.61 \mu \mathrm{g} / \mathrm{l})$, than in those without IMND $(0.14 \pm 0.06 \mu \mathrm{g} / \mathrm{l}, \mathrm{p}<0.001)$. Between infants with IMDN, 2 had a normal TnT-release $(<0.2 \mu \mathrm{g} / \mathrm{l})$, while 6 had an increased TnT-release. Infants with normal TnT-release had a mild and transient form of IMDN while infants with raised TnT-release had congestive heart failure or cardiogenic shock; two of these died. These results suggest that the release of CK and CKMB should nor be used as a marker of IMDN, while a raised level of $\operatorname{TnT}$ in the blood of newborns older than 28 gestational weeks are compatible with IMDN and suggest a poor outcome.
P-55

Influence of instrument setting on quantitative color Doppler flow mapping

Borm B, Will A, Hausdorf G, Lange PE

Department of Congenital Heart Disease, German Heart Institute, Berlin, Germany

Color Doppler echocardiography has been used to quantify valve regurgitation and ventricular septal shunt flow by measuring maximal jet area. However, the influence of instrument setting on these measurements has not been fully evaluated. We used a laminar flow model and a Siemens Sonoline SI 1200 ultrasound machine to measure jet area, length and mean velocity. We studied the influence on jet measurments of altering individually: (1) sector angle, (2) Nyquist Limit, (3) color map, (4) temporal and spatial resolution, and (5) digital filtering, from a basic setting. The effects on jet area are presented here: (1) Sector angle, $45^{\circ}$ to $30^{\circ}$, jet area reduced by $13 \%(\mathrm{p}=0.001)$; (2) Nyquist Limit, 40 to $60 \mathrm{~cm} / \mathrm{sec}$, jet area reduced by $26 \%(\mathrm{p}=0.001)$; (3) color map, $S 1$ to $S 2$, reduced by $30 \%(p=0.001)$; (4) resolution, largest change $34 \%$ $(p=0.001) ;(5)$ digital filtering, largest change $69 \%(p=0.001)$. The results for jet length and mean velocity were similar. In conclusion, instrument setrings have a large and significant influence on quantitative color Doppler flow mapping. Along with other clinical and technical variables, these factors make accurate quantification of flow with color Doppler echocardiography extremely difficult and prone to error.

\section{P-56}

Closure of patent ductus arteriosus by video-thoracoscopy in 70 children

\section{Lupoglazoff JM, Laborde F, Magnier S, Casasoprana A} Hôpital Robert Debre, Paris, France

For over 20 years, different methods of interventional catheterization have partially replaced surgical closure of PDA. We report the results of new operative technique, video-thoracoscopy, derived from endoscopic surgery. Under general anesthesia and after tracheal intubation, two trocars of $5 \mathrm{mn}$ diameter are introduced into the thorax for the passage of the instruments required for dissection and closure of the PDA. Two hooks are also introduced to retract the lung and dissect the ductal region. Two 9-mm titanium clips are positioned under videoscopic control. Seventy children underwent this procedure between February 1992 and July 1995 . The average age at the time of operation was 13.8 months (range 3 to 32 months) with an average weight of $14.5 \pm 5.5 \mathrm{~kg}$ (range 2 to $58 \mathrm{~kg}$ ) including $14(20 \%)$ with a body weight of less than $6 \mathrm{~kg}$. The surgical indications were hemodynamic in $27 \%$ of cases (large shunts with pulmonary hypertension) and prophylactic against endocarditis in $73 \%$ of cases. There were no operative fatalities. The immediate complications included: chylothorax ( 1 case) and left recurrent nerve paralysis ( 3 cases). A residual shunt was observed in 3 of the 70 cases $(4.3 \%)$. In one of these cases, a supplementary clip was effective in suppressing the residual shunt and another one spontaneous closer occurred one year after surgery. The residual shunt was resected after a second failure of clipping the duct in the last case. The final closure rate was $98 \%$. Closure of PDA by video-thoracoscopy is a rapid and safe technique. It can be used in premature children when endovascular methods are impossible and in systematic closure of large ducts in children with low body weights. 


\section{P-57}

Prognosis in young patients with hypertrophic obstructive cardiomyopathy

Azzano O, Bozio A, Sassolas F, Di Filippo S, Monrozier DJ Hôpital Cardiologique, Lyon, France

Forty pts in whom hypertrophic obstructive cardiomyopathy (HOCM) had been diagnosed before the age of twenty years by echo or catheterization were followed in the same institution and were studied retrospectively in order to assess the natural history and prognosis. Eighteen boys and 22 girls were 3 days to 19.9 years old (median 11.2 years) at time of the initial examination. A family history of HOCM and/or sudden death was present in $52 \%$. Twenty-one pts had symptoms-dyspnea NYHA class $3 \mathrm{II}$ (13 pts), chest pain (8 pts), palpitations ( $4 \mathrm{pts}$ ) and syncope (7 pts). Nineteen pts were asymptomatic. Thirtyfour pts received medical (beta blocker 32 pts, verapamil 1, amiodarone 2) or surgical treatment ( $7 \mathrm{pts}$ ). Six pts were followed without treatment. During a mean follow-up of 10.2-6.8 years (range 0.1-24.7 years), 13 pts died ( 2 of unknown cause, 1 non-cardiac and 10 cardiac deaths, among which 8 were sudden). Cumulative annual mortality was $2.3 \%$, and 5- and 10-year survival rates after diagnosis were 90 and $80 \%$ respectively. The 27 survivors were compared with the 13 deaths. Among clinical and hemodynamic features, only syncope was associared with global mortality $(p=0.004)$ and sudden death $(p=0.0008)$, but death is not well predicted by hemodynamic findings.

\section{P-58}

Experiences with the fourth generation of the buttoned device for interventional closure of atrial septal defects

Berger F, Nürnberg JH, Abdul-Khaliq H, Dähnert I, Ublemann F, Alexi-Meskishvili $V$, Sideris EB, Lange PE

German Heart Centre Berlin, Berlin, Germany, Athenian Institute of Peduatric Cardiology and Custom Medical Devices, Athens, Greece

Only recently was it possible to close atrial septal defects (ASD) by means of catheter technologies. Since 1992, in 71 pts ASD closure was performed with Sideris Buttoned Device (BD). The BD is a custommade investigational device with an occluder and a counteroccluder. The differences between the 4 th generation (gen) and the older gen were a double button for securing the device with the counteroccluder and reconstruction and stabilization of the struts of the occluder to avoid wire migration, which had been happening in earlier versions. Until July 1993, 2nd/3rd gen of BD were used in 31 pts and then ASD closure has been performed in 40 pts with the 4 th gen of $B D$. There were no significant differences for both groups in age, in body weight, in size of left-to-right shunt (1-r-s) and in size of ASD respectively.

\begin{tabular}{|c|c|c|c|}
\hline $4 \mathrm{th}$ & $\operatorname{sen}(n=40)$ & $2 n d / 3 r d$ gen $(n=31)$ & Total $(n=71)$ \\
\hline Full occlusion (\%) & 85 & 61.3 & 74.7 \\
\hline Trivial residual shunt (\%) & 2.5 & 16.1 & 8.5 \\
\hline Relevant residual shunt $\%$ & 2.5 & 6.5 & 4.2 \\
\hline Complications (\%) & 10 & 16.1 & 127 \\
\hline
\end{tabular}

The differences of all changes were highly significant. Complications were unbuttoning in $2.6 \%$ in 4 th gen and in $9.7 \%$ in the 2 nd and $3 \mathrm{rd}$ gen, migration in $0 / 6.5 \%$, device instability in $2.6 / 0 \%$ and trivial mitral incompetence in $5 / 0 \%$ respectively. These complications required surgical removal of the device and closure of the ASD by surgical intervention. The introduction of 4 th gen buttoned device significantly improved the results. The complication rate is acceptable and therefore the interventional closure of ASD with the BD is a reasonable method and could be an alternative to surgery for selected defects.

\section{P-59}

An isolated cardiac conduction disease maps to chromosome $19 \mathrm{q} 13$ de Meeus A, Stéphan E, Debrus S, Jean MK, Loiselet J, Weissenbach J, Demaille J, Bouvagnet P

CRBM, CNRS UPR 9008, Montpellier, Service de Génotypage, Généthon, Evry, France and Faculté de Médecine, Université St Joseph, Lebanon

Isolated Cardiac Conduction Disease (ICCD) is an autosomal dominant defect that includes various combinations of bundle branch or fascicular blocks. It expression starts as early as the first year of life. These defects can cause sudden death due to a complete heart block. The penetrance is incomplete and different between female and male (55\% and $75 \%$, respectively).We used a genome wide screening approach with polymorphic $(\mathrm{CA}) \mathrm{n}$ repeat markers to determine the chromosomal position of the gene defect implicated in this disorder. The analyses were carried out on a large Lebanese kindred which included individuals with either a complete or incomplete right bundle branch block (RBBB) with a vertical axis deviation $\left(<-30^{\circ}\right.$ or $\left.>+100^{\circ}\right)$. Linkage to the disease locus was detected with the polymorphic marker D19S604 on the $q$ arm of chromosome 19 (19q13.3) with a multipoint lod score of 7.18. Additionally, we were able to exclude the flanking loci D19S606 and D19S571 which are $13 \mathrm{cM}$ apart because of recombination events in 3 affected individuals. Two potassium channel genes are located in this region and we will present the result of their sequencing. In addition, a comparison of ECGs berween mutation carriers and non-carriers will be presented which allows a good prediction of the mutation status in borderline cases.

\section{P-60}

Pulmonary stents as a preparation for correction of tetralogy of Fallot

Brzezıbska-Rajszys G, KsiazykJ, Tynan M, Qureshı S, Zubrzycka M, Stodulski

Child Health Center, Warsaw, Poland and Guy's Hospital, London, United Kingdom

Three pts unsuitable for tetralogy of Fallot (TOF) repair had PalmazSchatz stents implanted in pulmonary arteries to become good surgical candidates. A 11 -year-old girl with TOF and hypoplastic left pulmonary artery (LPA) had a left BT shunt performed at 6 months of age (patent for 3 months) and right BT shunt 2 yrs later. Additional multiple peripheral pulmonary artery stenoses appeared on angiogram performed 4 years later. She was not accepted for surgical repair. Two pulmonary stents (right upper and lower pulmonary artery) and balloon dilatatıon of LPA as the first stage procedure were performed, complicated by hyperperfusion injury of the right lung. Six months later the third stent was implanted in LPA. After 3 months, correction of TOF was successfully performed. One 15-year-old boy had TOF with multiple peripheral pulmonary artery stenoses both natural and after previous bilateral BT shunts. He had stent implantation in both branch PA's with reduction of main PA pressure. He underwent successful correction and is well 6 months after. One 17-year-old girl had pulmonary atresia, VSD and collaterals. After numerous procedures (bilateral $\mathrm{B}$ T shunts, Brock valvotomy with balloon dilation of RVOT) bilateral branch PA stenoses were noted. She had stenting of both branch PA's with a fall in main PA pressure. She is well 7 months after correction of TOF. In conclusion, in pts with some forms of tetralogy of Fallot not suitable for surgery, stent implantation can be an effective way to prepare them for correction. 


\section{P-61}

The miniature high frequency transesophageal echocardiographic transducer-diagnostic value and safety of application in the small and sick patient

Cromme-Dijkhuis AH, Djoa KK, Bom N, Hess J

Pediatric Cardiology and Experimental Echocardiography, Sophia Children's Hospital, Erasmus University, Rotterdam, The Netherlands

A newly developed transesophageal transverse plane $7.5 \mathrm{MHz}$ transducer with tip dimensions of $17.5 \times 7 \times 5 \mathrm{~mm}$ and a gastroscope diameter of $5 \mathrm{~mm}$ was applied in 23 small pts with various congenital heart defects. Ages ranged from 2 days to 1 year and weights from 1.8 to $7 \mathrm{~kg}$. Transesophageal echocardiography (TEE) was applied when transthoracic echocardiography (TTE) only showed poor quality imaging because of lung disease ( 3 pts), thoracic cage deformity $(5 \mathrm{pts})$ or artificia ventilation ( $5 \mathrm{pts}$ ). TEE also was performed when TTE left questions about the exact anatomy of the heart. In the 23 pts, the morphology and/ or function of 47 cardiac lesions or structures were determined by echocardiography. TEE proved to be superior to TTE in the assessment of 7 lesions in 5 pts, validared by cardiac catheterization or surgery. TEE provided additional information to TTE of 14 lesions in 12 pts. Especially in more complex heart defects, this information sometimes was crucial for proper management. Particularly in this population, the pulmonary venous return, the superior part of the atrial septum, the morphology and function of atrioventricular valves in complex heart disease and the nature of left and right ventricular outflow tract obstructions were solely or better defined by TEE in comparison to TTE. The TEE studies in these pts were safe procedures as no complications were encountered, even in sick infants. In conclusion, this miniature transesophageal transducer has definite merit in defining the morphology of congenital heart defects, in particular in small infants in the neonatal intensive care in which transthoracic imaging is not adequate. TEE with this transducer is a safe procedure, even in sick infants.

\section{P-62}

Growth of the cardiac allograft after heart transplantation in children Di Filippo S, Sassolas F, Ninet J, Champsaur G, Bozio A Hôpital Cardio-Vasculaire Louis Pradel, Lyon, France

Growth of the cardiac allograft specifically concerns pediatric recipients. We reviewed retrospectively echocardiographic data from our dinical series in order to address this issue. From March 1987 to May 1994, 17 pts less than 13 years of age were transplanted, and 11 of them $(6$ children and 5 infants) have been followed for at least 18 months (18 months to 8 years, mean 3.5 years). These 11 recipients were aged 2 days to 13 years (mean 6.5 months) at surgery. The age of the donor ranged from 7 days to 13 years (mean 4 years) in 10 cases and one donor was 27 years old. Donor-to-recipient weight ratio ranged from 0.83 to 5 (mean 1.75). All recipients received triple immunosuppressive therapy (cyclosporin, azathioprin, continuous steroid therapy). Echocardiographic data were collected monthly after the third postoperative month. Linear body growth was normal in 8 cases, and retarded in 3 cases. Mean body surface area (BSA) increased from 0.68 to 0.94 during follow-up. There were no episodes of acute rejection nor elevated blood pressure. Left ventricular (LV) diameters and LV mass increased linearly with BSA. Velocity of growth was maximal in the infant group, reaching $18.3 \%$ annually. Early postoperative elevated LV mass index (mean $168.2 \mathrm{mg} / \mathrm{m}^{2}$ ) returned to normal range within the first 6 months $\left(100-125 \mathrm{mg} / \mathrm{m}^{2}\right)$. LV indices remained within normal limits when compared with published standard heart growth curves (at the upper limit in infants). In our experience, denervared transplanted heart grows linearly with BSA, and this growth is similar to that of normally growing children's hearts.
P-63

Balloon valvuloplasty through the right carotid artery for critical aortic stenosis in newborns

Guccione P, Formigari R, Attisano T, De Simone G, Ballerini L Pediatric Cardıology, Ospedale Bambino Gesù, Rome, Italy

The purpose of this study was to retrospectively assess the efficacy and safety of the RCA approach for the percutaneous treatment of critical aortic stenosis in newborns. Between August 1990 and September 1995,15 consecutive newborns (mean age $1.6 \pm 0.3$ months, mean weight $3.6 \pm 1.2 \mathrm{~kg}$ ) were submitted to percutaneous balloon dilatation (PBD) of a critically stenotic aortic valve, with vascular access through a surgically exposed RCA. Two pts had had a previous attempt through the right femoral artery without success. Dilatation was carried out with the usual balloon technique. Transvalvular peak systolic gradient was reduced from a mean of $64 \pm 16 \mathrm{~mm} \mathrm{Hg}$ to a mean of $20 \pm 12 \mathrm{~mm} \mathrm{Hg}$ $(p<0.05)$. Subsequent aortic regurgitation was absent or trivial in $7 \mathrm{pts}$ (47\%), moderate in 6 pts (40\%) and severe in 2 pts (13\%). There was one in-hospital death (with moderate aortic regurgitation) and three late deaths, respectively at 3,4 and 5 months after the procedure, all due to persistent left ventricular dysfunction. In three pts, respectively 3,5 and 7 months after PBD, a second procedure has been performed through a femoral artery because of the recurrence of a significant left ventricleaortic pressure gradient. In conclusion, PBD through the RCA is a safe and effective technique and should be regarded as the first choice for palliation of critical aortic stenosis in newborns.

P-64

Is cardiac Troponin- $T$ a good marker for assessment of cardiac injury in children?

Kaku S, Miranda F, Pachica F, Pinto F, Trigo C Serviço de Cardiologia Pediatrica, Hospital de Santa Marta, Lisboa, Portugal

To eval uate the efficiency of cardiac Troponin $\mathrm{T}(\mathrm{cT} \mathrm{Tr})$ for the detection of myocardial cell damage in children (ch) with CHT, 32 consecutive pts were prospectively enrolled. Five groups: I (control group)-7 pts nor submitted to interventional catheterization (cc) or surgery; II $-6 \mathrm{ch}$ submitted to cc; III-8 ch submitted to closed cardiovascular operations; IV-6 pts with open heart surgery; V-5 ch with complete transposition of the great arteries submitted to Jatane operation. Groups I and II had no clinical evidence of myocardial ischemia. In group I, one blood sample for each pt was obtained; in the other groups, 6 hourly samples were drawn (four in groups II and III and seven in groups IV and V). In all blood samples, total creatine kinase (CK-IU/l), MB isoenzyme of CK (MBCK-percentage of CK) and cTnt $(\mathrm{ng} / \mathrm{ml})$ were measured.

$\begin{array}{lccccc}\text { Group } & \text { I } & \begin{array}{c}\text { II } \\ \text { (peak) }\end{array} & \begin{array}{c}\text { III } \\ \text { (peak) }\end{array} & \begin{array}{c}\text { IV } \\ \text { (peak) }\end{array} & \begin{array}{c}\text { V } \\ \text { (peak) }\end{array} \\ \text { CK } & 56-1053 & 151-2508 & 322-14975 & 725-3162 & 319-2583 \\ \text { MBCK } & 14-66 & 4-23 & 1.4-45 & 5.6-14 & 5.8-27 \\ \text { cTnt } & 0.05-0.29 & 0.08-1.28 & 0.35-49.9 & 4.4-14.1 & 3.4-40\end{array}$

Group I samples and the first sample of group II pts (drawn before cc) had cTnt values $<0.29 \mathrm{ng} / \mathrm{ml}$ (controls). Group II cTnt values were normal before 18 hours post cc. In Group III, peak values were obtained 6-12 hours after surgery. In 3 pts of group IV not submitted to ventriculotomy, peak values were reached 18 hours after surgery. The highest $c$ Tnt peak value in group $V$ was obtained in a child with an intramural coronary artery that was damaged during the operation. In conclusion, $\mathrm{CK}$ and MBCK values could not detect myocardial injury in most of ch studied. Measurement of $\mathrm{cTnt}$ assessed accurately myocardial damage during $c c$ or cardiac surgery. 
P-65

Right ventricular diastolic dysfunction after Fallot repair

Krogmann O, Kromeke O, Heusch A, Rubo J, Bourgeois M

Pediatric Cardiology, Heinrich Heine University, Düsseldorf, Germany

Tetralogy of Fallot (TOF) leads to significant right ventricular (RV) pressure and volume overload with possible long-lasting effects on RV diastolic function. However, data on right ventricular passive elastic properties in pts with TOF are limited. Therefore, 12 pts (age 5.8 \pm 4.7 years) were studied to assess RV systolic and diastolic function after TOF repair. Biplane RV angiocardiography and simultaneous high fidelity pressure measurements were obtained in 6 pts with TOF postoperatively and 6 pts with only minimal heart disease (C). LV and RV volumes were determined frame by frame by the multiple slices technique and normalized for end-diastolic volume. Systolic function was assessed from ejection fraction (EF) and diastolic function from the diastolic pressure-volume relationship using a three constant elastic model (normalized chamber stiffness constant: b). RV ejection fraction was depressed in pts with TOF when compared to C ( 48 vs $65 \%$ in C). The diastolic pressure-volume relationship was shifted upwards and to the left in pts with TOF; b was increased in pts with TOF (11.5 vs 4.4 in $\mathrm{C} ; \mathrm{p}<0.05$ ). In conclusion, $\mathrm{RV}$ ejection fraction is reduced after surgery for TOF, probably due to the RVOT patch and the presence of pulmonary regurgitation. $\mathrm{RV}$ diastolic function is also impaired, possibly as a consequence of preoperative pressure overload.
P-67

Influence of cholesterol screening and nutritional counseling in reducing cholesterol levels in children

Moodie DS, Garcia R

The Cleveland Clinic Foundation, Cleveland, United States of Amenca

To determine whether cholesterol screening and nutritional counseling can reduce cholesterol concentrations in populations of otherwise unrecognized hypercholesterolemic children. The present study examines data from a cohort of 894 children ( 473 boys, 421 girls) who had cholesterol concentrations about $185 \mathrm{mg} / \mathrm{dl}$ (the 90 th percentile) at baseline, and after counseling had a repeat measurement an average of 2.2 years later. Their mean ages were 7 years at the first testing and 9.2 years at the second. Children who had cholesterol concentrations above $200 \mathrm{mg} / \mathrm{dl}$ (the 95th percentile) had lipoprotein profiles done, and if their LDL cholesterol were elevated were referred to a nutritionist, and family members were advised to have their blood lipids analyzed. Mean cholesterol concentration of all 894 children over this time period decreased by $9.4 \%(19.5 \mathrm{mg} / \mathrm{dl} ; 95 \% \mathrm{Cl}=17.5 \mathrm{mg} / \mathrm{dl}$ to $21.5 \mathrm{mg} / \mathrm{dl}$; $\mathrm{p}=0.001$ ) was observed for the 463 children who had initial cholesterol concentrations between 185 and $200 \mathrm{mg} / \mathrm{dl}$ and who therefore received a less intense intervention. In conclusion, cholesterol concentrations in groups of otherwise unidentified hypercholesterolemic children can be significantly reduced as a result of cholesterol screening and nutritional counseling in a pediatric practice setring.

\section{P-66}

Congenital heart disease associated with chromosome 22q11 deletion

Momma K, Kondo C, Matuoka R, Takao A

Tokyo Women's Medical College, Tokyo, Japan

The purpose of this study was to clarify characteristics of congenital heart disease associared with chromosome $22 \mathrm{q} 11$ delerion. Chromosome $22 q 11$ deletion causes congenital hearr disease and other anomalies included in DiGeorge, velo-cardio-facial, and conotruncal anomaly face syndromes. One hundred cardiac pts were identified as having chromosome 22q11 deletion, which was confirmed by FISH with the Oncor N25 probe. Cardiovascular anomalies were studied with echocardiography and angiocardiography. Tetralogy of Fallot was present in 73 cases, associated with pulmonary atresia in 29 . Associated aortic arch anomalies were usual. Isolated ventricular septal defect was present in 12 cases, and was perimembranous in 10 and infundibular in 2. Type B interrupted aortic arch was present in 4 cases. Double outlet right ventricle was present in 3 cases. Truncus arteriosus was present in 2 cases. Isolated aortic arch anomalies were present in 5 cases-isolated innominate artery; isolated right subclavian artery; right aortic arch, right ductus arteriosus and left pulmonary artery stenosis; high, stenotic right aortic arch and left descending aorta;high right aortic arch and right patent ductus arteriosus. In conclusion, chromosome $22 \mathrm{q} 11$ deletion causes a wide spectrum of congenital heart diseases. Associated tetralogy of Fallor with aortic arch anomalies is characteristic.
P-68

A new method to determine pulmonary hypertension in congenital heart diseases

Olguntürk R, Yener A, Ünlü M, Guinaydın S, Tunaoglu S, Özdogan ME, Gökgöz L, Halit V, Soncul H Gazi University, Medical Faculty, Ankara, Turkey

The indication and timing of the operative procedure for congenital heart defects are critical, since pulmonary artery pressure (PAP) and pulmonary vascular resistance (PVR) are the primary impediments to a successful outcome. We studied the diagnostic role of Tc-99m HMPAO lung clearance in pediatric pts with congenital heart defects having mainly left-to-right shunts undergoing surgery $(30 \mathrm{pts}$ undergone surgery and 6 pts not operated) and correlated the results with hemodynamic data obtained during preoperative recent catheterization and pathological classification of the specimens obtained during the operation as well. Lung tissue preparations were evaluated with respect to the degree of PHT in 3 grades; 1-2, 3 and 4 histopathologically. According to the pathological grading, very well-correlated results were obtained $(t 1 / 2$ values and PAP, PVR).

$\begin{array}{lcccc} & t^{1 / 2} & \text { PAP } & & \text { PVR } \\ \text { G 1-2 } & 8.37 \pm 9.0 & 24.08 \pm 3.99 & r=0.86 & 2.34 \pm 3.6 \\ \text { G 3 } & 16.78 \pm 2.49 & 50.67 \pm 6.36 & r=0.86 & 4.60 \pm 0.86 \\ \text { G 4 } & 38.5 \pm 3.73 & 69.5 \pm 4.09 & r=0.87 & 6.03 \pm 0.19 \\ \text { Inop G } & 49.3 \pm 4.8 & 90.67 \pm 4.13 & r=0.86 & 7.04 \pm 0.1\end{array}$

As a result, Tc 99m-HMPAO lung clearance seems to be a useful and challenging parameter for the non-invasive evaluation and monitoring of pulmonary hypertension, especially in infants for the decision of surgery and follow-up. 


\section{P-69}

Management of hypoplastic left heart syndrome-early experience Hansen PB, Lauridsen P, Jacobsen JR, Pryds O, Pettersson G Rigshospitalet, University of Copenhagen, Copenhagen, Denmark

To present our initial experience with staged operative palliation for hypoplastic left heart syndrome (HLHS), from May 1993 to October 1995, we have performed thirteen stage 1 and seven stage 2 procedures in thirteen pts with HLHS. Neonates were resuscitated from systemic hypoperfusion using prostaglandin and judicious ventilatory adjustments, aiming to preserve pulmonary vascular resistance and a pulmonary-to-systemic flow ratio near uniry. Aortic arch reconstruction was performed using homograft material and incorporation of the native ascending aorta in 7 cases. In 6 cases, a direct pulmonary artery-to-arch reconstruction, leaving the native ascending aorta as a coronary conduit, was employed. Systemic-to-pulmonary conduits were established using 3.5 or $4 \mathrm{~mm}$ Gore-Tex shunts. Stage 2 consisted of a bidirectional Glenn anastomosis. The postoperative course after stage 1 was stormy; low cardiac output, pulmonary-to-systemic flow imbalances and renal failure were common problems. Three pts died in hospital following stage 1; two pts died within 24 hours of surgery due to circulatory failure and systemic/pulmonary flow imbalance and the third 18 days postoperatively due to low cardiac output and multiorgan failure. Nine pts survived stage 1 and were discharged; but one of these died from pneumonia at 2 months of age. Postoperatively, stage 2 was less stormy, although problems with low pulmonary blood flow and cardiac output did arise. Seven pts have undergone stage 2; five were discharged. One (in whom TCPC was attempted) died 4 weeks after surgery from multiorgan failure and one (with small bilateral caval veins) died 18 days postoperatively from low cardiac output and low saturation. Thus, a total of 7 of 13 pts are still alive, two awaiting stage 2 and five stage 3 . In conclusion, our results, representing an early learning curve, show that HLHS pts are difficult to treat. Even in a small center, treatment is possible with acceptable results.

\section{P-70}

The natural history of left ventricular hypertrophy in preterm neonates associated with short course dexamethasone

Skelton S, Parsons J, Gill B

Peter Congden Neonatal Unit, Leeds General Infirmary, Leeds, United Kingdom

The natural history of left ventricular hypertrophy (LVH) associated with dexamethasone (dexa) treatment for chronic lung disease was studied in 24 neonates, gestation 23-34 weeks, birth-weight 500-2054 g. All received dexa $(0.4-0.6 \mathrm{mg} / \mathrm{kg} /$ day reducing over $15-23$ days $)$. Thirty-six neonates matched for gestation and age were studied as controls. Baseline and serial echocardiographic studies up to a maximum of 48 days were performed. The left ventricle (LV) was studied from cross-sectional, parasternal long-axis views. M-mode measurements of end-diastolic interventricular septum (IVS) and LV posterior wall (LVPW) thickness were taken at the level of the mitral valve leaflets, expressed as percent change from baseline and compared to control values. Distribution of LVH was assessed from serial short-axis views of the LV from the mitral valve leaflets to the apex. Continuous and pulsed wave Doppler velocities were taken from the LV outflow tract (LVOT). LVH occurred in 23/24 babies. It was first seen by day 2-11 of dexa. Four babies died by day 5 of lung disease. LVH progressed to maximum by day 4-25 in 18 babies. Maximum IVS thickness was $80 \pm 37 \%$ and LVPW $73 \pm 37 \%$ greater than baseline. Twelve babies had asymmetrical septal hypertrophy. No localized segmental LVH was seen. In 11 babies, studied to 48 days from starting dexa, complete resolution of LVH occurred by 15-32 days. In 13 babies peak LVOT Doppler velocities were $0.5-1.75 \mathrm{~m} / \mathrm{sec}$. Ejection systolic murmurs occurred in 2 babies, 1 developed cardiac failure which quickly resolved. Severity of LVH appeared unrelated to blood pressure, gestation or birth-weight. LVH is likely to develop in almost all preterm babies given dexa. Symptoms and echo features similar to familial hypertrophic obstructive cardiomyopathy may occur but should resolve.
P-71

The genetic relation between blood pressure and heart rate responses in isometric and mental stress tests-study in twins before puberty

\section{Verhaaren HA, Matthys D, De WolfD, Schieken RM \\ University Gent, Gent, Belgium and VCU-MCV, Richmond, United States of America}

Heart rate and blood pressure responses to isometric stress (handgrip test) and to mental arithmetic were studied in ninety-five 11-year-old twins. The aim of the study was to investigate whether the relation between these two stress indices is at least partly based on genetically determined mechanisms. Univariate and bivariategenetic analysis of the data were performed using parh analysis and maximum likelihood statistics (LISRELR). Univariate genetic analysis showed a high genetic variance (around $80 \%$ of the total variance) for heart rate in both tests. For systolic blood pressure, genetic variance was only found in mental arithmetic testing. In the bivariate genetic analysis, no model searching for a relation of heart rate or systolic blood pressure between the two stress conditions reached statistical significance. However, models looking for a relation between heart rate and systolic blood pressure within the same test condition were statistically significant. Furthermore, the covariance (variance common to both variables) was for more than $60 \%$ of genetic origin. Therefore, we conclude that the relation of heart rate and systolic blood pressure in both stress conditions is partly explained by common mechanisms which are genetically determined. The mental arithmetic and isometric handgrip tests are clearly independent assessments of cardiovascular reactivity based on totally different regulatory mechanisms. These stress tests may be helpful in the exploration of the blood pressure regulating mechanisms in the pediatric age group.

\section{P-72}

Thrombosis and embolism after modified Fontan operation risk factors and anticoagulation

von Arnim V, Sauer U, Stern H, Kirchner I, Malcic I, Lorenz H-P, Büblmeyer $K$

Kinderkardiologie, Deutsches Herzzentrum, München, Germany

Previously, thrombosis and embolism have been considered as rather rare complications after modified Fontan operation (MFO). In a retrospective study, we assessed the circumstances, that might be a cause or a predictor of thromboembolic events. Our interest was focussed on a subgroup of 25 pts who underwent MFO. MRI was performed in all pts in order to evaluate their postoperative status with respect to the size of anastomoses, flow pattern within the right atrium and evidence of thromboses. Of 21 thromboses or embolisms, 17 could be ascertained in 11 pts by MRI (10) or other first diagnostic means (echo 7, cath/angio 3 , CT 1), and 4 such formations in 3 pts were highly suspective. Five pts had 2 to 3 recurrences of thromboses. Twelve pts had no evidence of thromboembolic events. The mean interval between MFO and the diagnosis of thrombosis was 45 months (range 7 days-168 months). Only 3 thrombotic complications occurred during anticoagulation with warfarin or heparin, all the remaining were diagnosed after anticoagulation therapy had been discontinued. In these latter pes, all coagulation studies had been normal. Furthermore, the variability of clinical signs, inconsistency of diagnosis when different diagnostic means were used, the lack of predictive laboratory results and a less strict protocol for postoperative anticoagulation made it difficult to estimate the risk for thromboembolic complications in our pts. Based on our study results and in accordance with the experience of other centers, a protocol for anticoagulation after MFO is proposed, which relates to high risk and low risk pts after MFO. 
P-73

Early and mid-term follow-up after repair of tetralogy of Fallot in adults

Dähnert I, Dittrich S, Ublemann F, Berger F, Weng Y, Alexi-Meskishvili V, Hetzer R, Lange PE

German Heart Center, Berlin, Germany

The aim of this retrospective study was to assess early and mid-term outcome of adults undergoing repair of tetralogy of Fallot (TOF). Between 1988 and 1994, 27 adults (ages 17 to 54 years) underwent complete repair for TOF. Four pts received primary repair. Eight pts had received previous palliation (systemic-pulmonary shunt or Brock pulmonary valvotomy) mean 20.8 years before correction. Fifteen pts underwent reoperation mean 14.5 years after previous corrective repair. Indications were severe cyanosis (12), severe pulmonary insufficiency (13), right ventricular dysfunction (22), large residual ventricular septal defect (3) or intolerable arrhythmia (5). Eighteen pts received a pulmonary homograft. A mechanical valve had to be implanted in 2 cases. Two pts required additional reconstruction of tricuspid or aortic valve, respectively. One $\mathrm{pt}$ had additional aortic valve replacement. Three $\mathrm{pts}$ $(12 \%)$ died in-hospital. There were no late deaths. Twenty-four pts were followed to 1995 for a mean of 24 months (range 6 to 76 ). NYHA status improved in $18 \mathrm{pts}(75 \%)$ and did not change in $6 \mathrm{pts}(25 \%)$. At the time of the last examination $9(38 \%), 11(45 \%)$ and $4(17 \%)$ pts were in NYHA class I, II and III, respectively. All pts stated postoperative improvement in quality of life. Hemodynamic evaluation showed mild pulmonary stenosis in 11 pts (45\%) and pulmonary insufficiency in 14 pts (58\%) (11 mild, 3 moderate). Arterial $\mathrm{SO}_{2}$ was $>94 \%$ in all pts. Nine pts are on medical treatment for supraventricular $(5 ; 21 \%)$ or ventricular (4; 17\%) arrhythmias, none of them considered life-threatening. In conclusion, repair of TOF in adults is possible with acceptable risk. Early and mid-term results suggest improvement in hemodynamic parameters as well as in qualiry of life. The incidence of arrhythmias was nor reduced. Late outcome and risk of sudden death remain to be evaluated.

\section{P-74}

Follow-up of balloon dilatation for native coarctation

Filippini LHPM, Beekman RP, op de Coul BMR, Beek FJA, Hutter PA, Meijboom EJ

Departments of Cardiology and Radiology, Wilhelmina Children's Hospital, Utrecht, The Netherlands

The aim of this study was to evaluate MRI measurements as a tool for diagnosis, choice of therapeutic approach and follow-up of coarctation of the aorta. Fifteen children were examined before and after balloon angioplasty for native coarctation with MRI, 2D-echo and blood pressure measurements (BPM). Results were compared with data obtained at cardiac catheterization (CC). Ages ranged from 0.4 to 14.9 years. MRI studies were performed 1-2 months before and 2-24 months after dilatation. The ratio of area of the aorta at coarctation side: area of the aorta at diaphragm level was obtained by two independent observers. Interobserver variability was not significant $(p>0.5)$. The data were compared by linear regression analysis and paired $t$-test. Echo Doppler gradients ranged from 55 (range 23-80) $\mathrm{mm} \mathrm{Hg}$ before to 24 (range 10 50) $\mathrm{mm} \mathrm{Hg}$ after plasty, BPM gradients ranged from 41 (range 31-60) $\mathrm{mm} \mathrm{Hg}$ to 23 (range 2 to 52 ) $\mathrm{mm} \mathrm{Hg}$. Correlations between gradients measured at $\mathrm{CC}$ and Echo $(\mathrm{r}=0.81, \mathrm{p}<0.001)$, and $\mathrm{CC}$ and $\mathrm{BPM}$ $(r=0.54, p<0.002)$ were obtained. Correlation between diameter obtained at the coarctation side at MRI and Angio was high ( $r=0.96$, $\mathrm{p}<0.001$ ). All pts with BPM-gradients of $25 \mathrm{~mm} \mathrm{Hg}$ or more indicating significant coarctation showed MRI-ratios $<0.4$ (area reduction at coarctation side $\geq 60 \%$ ) indicating significant coarctation, while all pts with BP-gradients $<25 \mathrm{~mm} \mathrm{Hg}$ gave MRI ratios $>0.4$ indicating insignificant coarctation. Two pts developed recoarctation demonstrated either by a blood pressure gradient $>25 \mathrm{~mm} \mathrm{Hg}$ or a MRI ratio $<0.4$. We conclude that MRI provides an excellent tool for diagnosis and follow-up of coarctation due to its ability to accurately visualize the anatomy of the aorta. MRI is of specific importance for the exclusion of the presence of postdilatation aortic aneurysms.

\section{P-75}

Clinical aspects before and after surgical therapy of Ebstein's anomaly Nagdyman N, Ewert P, Ublemann F, Hetzer R, Lange PE German Heart Institute Berlin, Berlin, Germany

Do pts with Ebstein's anomaly benefit from surgical treatment? We reviewed the clinical and surgical results in children and adults presenting with Ebstein's anomaly. From October 1988 to September 1995, 36 pts ( 18 female, 18 male) were examined by cardiac catheterization and echocardiography. Ages ranged from the first day of life to 65 years (mean 26 years). Eighty-three percent had an additional atrial septal defect or open foramen ovale. Ten pts $(28 \%)$ had other associated congenital heart defects. We observed 3 pts with an ischemic stroke, 4 had a transient ischemic attack, 2 had a syncopal episode and one complained of occasional dizziness. Twenty-eight pts (13 females, 15 males) underwent surgery on the basis of clinical symptoms as paradoxical emboli, progressive cyanosis and functional class III or IV symptoms. In $90 \%$ of pts, closure of associated atrial septal defect was done; $72 \%$ received tricuspid valve reconstruction and $18 \%$ a prosthetic valve. One pt underwent surgical treatment as aortic arch reconstruction for interrupted aortic arch and another a BT shunt operation for subatretic pulmonary atresia. Four pts died within the early postoperative period (14\%). Clinical and echocardiographic follow-up periods were 1 to 62 months (mean 23 months). Nineteen pts showed clinical improvement, 17 by one functional class, two by two classes. Five pts had no changes in functional class. Tricuspid regurgitation improved in 21 pts, in 9 pts by one grade, in 12 pts by two grades. Five pts had no changes in tricuspid regurgitation. Echocardiographic data were missing in 2 pts. In conclusion, patients with Ebstein's anomaly benefit from surgical treatment. They show significant improvement in functional class and reduction in severity of tricuspid regurgitation.

\section{P-76}

End-to end resection for isolated coarctation-comparison of results in neonates and infants

Pfammatter J-P, Ziemer G, Kallfelz $H C$

Pediatric Cardiology and Cardiothoracic Surgery, Hannover, Germany

Optimal timing for surgical relief of aortic coarctation is still debated. A retrospective study was undertaken to compare the results of end-to-end resection for isolated coarctation in 46 pts operated on by the same surgeon during a seven-year period ending in 1993 . Twenty-six neonates were operated on an emergency basis at a mean age of $12 \pm 8$ days with a mean weight of $3.5 \pm 0.5 \mathrm{~kg}$ (range $2.7-4.5 \mathrm{~kg}$ ). Twenty pts had elective repair in infancy at a mean age of $4.7 \pm 3$ months (range $2-11$ months) with a mean weight of $6 \pm 1.7 \mathrm{~kg}$ (range $3.6-9.5 \mathrm{~kg}$ ). Mean aortic cross-clamp time was $18 \pm 6 \mathrm{~min}$ in the neonates as compared to $14 \pm 3$ minutes in the infants $(\mathrm{p}=0.02)$. Surgical mortality was zero in the whole population. Except for one pt with chylothorax, there were no serious complications. Mean postoperative blood pressure gradient did not differ statistically between the two groups $(10 \pm 7 \mathrm{~mm} \mathrm{Hg}$ in the neonates versus $7 \pm 9 \mathrm{~mm} \mathrm{Hg}$ in the infants). During a mean follow-up of 49 months (range 13-95 months), recoarctation (blood pressure gradient $>20 \mathrm{~mm} \mathrm{Hg}$ ) was diagnosed in 5 pts with neonatal surgery (19\%) as compared to $1 \mathrm{pt}$ with surgery in infancy $(5 \%)$. Of these 6 pts with recoarctation, 4 had a reintervention (1 surgery, 3 balloon dilation), 2 pts had not to be treated so far. Recoarctation occurred within the first 5 months after initial repair in all 6 pts. During the whole follow-up period, 11 pts (24\%) developed systolic right arm pressures $>90$ th percentile of normals ( 7 neonates, 4 infants, $p=n s$ ) but none needed treatment so far. Neonatal surgery for coarctation carries a considerable risk of recoarctation. Based on the data presented, it is concluded, that outside the neonatal period, elective repair for coarctation can be performed with a reasonably low risk for recoarctation. 
P-77

Early results of modified Norwood surgery for hypoplastic left heart syndrome in Göteborg

Südow G, Mellander M, Andreasson S, Sunnegärdh J, Grönstedt A, Berggren $H$

Departments of Thoracic Surgery, Pediatric Cardiology and Pediatric Anesthesiology, Östra Sjukhuset, Goteborg, Sweden

After an initial experience with Norwood surgery for HLHS in 5 infants who all died after stage I, we modified the surgical technique. After increasing the length of the posterior circumference of the pulmonary trunk with a pericardial patch, the pulmonary trunk was anastomosed to the ascending aorta at a level of about half its length, thereafter continuing the anastomosis to the aortic arch and completing the reconstruction over the coarctation area with a pericardial patch of proper size. In 2 cases we have even resected the ascending aorta and reanastomosed into the pulmonary trunk. Using this modified Norwood approach, 10 pts with HLHS have undergone stage I since July 1994 (group A). During the same time period, 5 infants with other complex heart disease were operated on with the same technique (group B). In group $A(n=10)$, the 30-day survival after stage I was $90 \%(9 / 10)$; one pt died 3 months after stage I and 5 have undergone stage II. The 30 -day survival after stage II was $100 \%$. One pr died 3 months after stage II. The causes of death were a narrow anastomosis between the ascending aorta and the arch in 2 pts and severe rightventriculardysfunction in one. In group $B(n=5)$, there is no mortality so far and 2 pts have undergone stage II. For all $\mathrm{pts}(\mathrm{n}=15)$, the median number of days on the ventilator after stage I (extubated pts, $n=14$ ) was 7 (range 3-38) and the median number of days in the ICU was 14 (range 4-76). Three pts were reoperated because of aortic obstruction and one of them also because of overshunting. Out of those 5 pts that have reached an age of more than 10 months, two have a head circumference less than -2SD. One of these two pts was small for gestational age at birth. In conclusion, the early results of modified Norwood surgery for HLHS in Göteborg seem promising so far.

\section{P-78}

Noninvasive cerebral oxygen delivery assessment in children with syncope during tilt test

Fuster M, Rodriguez-Núñez A, Errís J, Alonso C, Couceiro J, Hermuda A, Martinón JM

Hospital General de Galicia, Santiago de Compostela, Spain

The aim of this study was to assess the cerebral oxygen delivery in children with syncope and its relation to hemodynamic changes during tilt testing. Upright tilt test was performed in 10 children $(7$ girls and 3 boys, aged between 11 and 15 years) with suspected diagnosis of syncope and previous normal cardiological evaluation. Besides electrocardiographic, blood pressure, and peripheral arterial saturation monitoring, continuous noninvasive measurement of cerebral oxygen saturation (COS) was performed with near infrared spectroscopy. All tilt tests were positive ( 6 cardioinhibitory response and 4 mixed response). Basal heart rate was $89 \pm 16 \mathrm{bpm}$. Minimal heart rate ranged between 0 and $75 \mathrm{bpm}$. Basal COS was $73 \pm 4 \%$. In all cases a decrease of COS was detected at the onset of clinical manifestations and during syncope. COS was significantly lower $(68 \pm \% 3)$ than basal $\operatorname{COS}(p=0.01)$ at the interval to the start of the children's complaints. The difference was even more important when the minimum COS obtained during syncope (5\% $7 \%$ ) was compared with basal values ( $p=0.0001)$. In 6 cases the decrease in $\mathrm{COS}$ heralded changes in heart rate and blood pressure. Shortly after moving the children to supine position, $\operatorname{COS}$ values returned to basal levels. Changes in COS correlated with heart rate modifications, but not with peripheral arterial oxygen saturation. In conclusion, syncope in children is accompanied by a significant and precocious impairment of cerebral oxygen delivery that resolves with a return to the supine position. The $C O S$ can be monitored noninvasively and could improve tilt test sensitivity, perhaps preventing undesirable hemodynamic changes.
P-79

Reference values of pulmonary blood volume as a measure of hypoplasia of the pulmonary vascular system

Krings $G$, Berger $F$, Nitrnberg JH, Lange PE

Department of Congenital Heart Diseases, Deutsches Herzzentrum Berlin, Berlin, Germany

When choosing a suitable procedure for surgical therapy of congenital heart disease with hypoperfusion of single or several lung segments, information about the degree of hypoplasia of the pulmonary vascular system or of the pulmonary blood volume (PBV), respecrively, is of major importance. For this reason we examined reference values of PBV in 33 children suffering from congenital heart disease with normal lung perfusion. The age of the pts was $0.3-24.3(10.0)$ years, body surface area (BSA) 0.29-2.03 (1.15) $\mathrm{m}^{2}$, body weight (BW) 5-80 (36) kg. After injection of a bolus of indocyanin green $\left(4-10 \mathrm{ml}, 4^{\circ} \mathrm{C}, 1 \%\right)$ into the pulmonary artery, we continuously measured the change in dye concentration and in temperature via an aortic Fiberoptic Thermistor Catheter (Cold System Z 021 Pulsion, Munich). We calculated the mean of PBV/ $\mathrm{BW}[\mathrm{ml} / \mathrm{kg}]$ and PBV $/ \mathrm{BSA}\left[\mathrm{ml} / \mathrm{m}^{2}\right]$ from three measured values of each pt. Results: BSA vs PBV, $y=-41.64+174.86 \cdot x ; r=0.92$ and for $B W$ vs $\mathrm{PBV}, \mathrm{y}=13.74+4.01 \cdot \mathrm{x} ; \mathrm{r}=0.93$. In conclusion, the thermodyne dilution method seems to be suited for finding reference values of $\mathrm{PBV}$ in the area of BSA from $0.29-2.03 \mathrm{~m}^{2}$ or BW from $5-80 \mathrm{~kg}$ respectively. The clinical relevance is at present being evaluated in children with a hypoplastic pulmonary vascular system.

P-80

Fetal cardiac and extracardiac circulations throughout pregnancya prospective longitudinal Doppler study

Matzas A, Areias JC, Barros $H$, Montenegro $N$

Department of Gynecology and Obstetrics, Paediatric Cardiology Unit, Epidemiology and Hygiene Department, University Hospital of San Joäo, Porto, Portugal

A longitudinal evaluation of cardiac and extracardiac blood flow velocity waveforms was performed in 30 healthy fetuses from 10 weeks gestation to term by using both transvaginal and transabdominal color and pulsed Doppler ultrasonography. Recordings were obtained from the fetal umbilical artery (UA), middle cerebral artery (MCA), aorta (Ao), and at the level of atrioventricular (AV) valves in four predetermined periods: $10-13,20-24,30-32$ and 35-37 weeks gestation. Fetal heart rate decreased significantly from the 1 st $(167.3 \pm 6.9 \mathrm{bpm})$ to the 2nd (1 $40.0 \pm 7.7 \mathrm{bpm}$ ) trimester of pregnancy (TP). Peak systolic velocity of UA, MCA and Ao increased significantly from the 1 st to the 3rd TP. In early pregnancy, end-diastolic flow was almost always negligible in the UA and Ao; it was present in the MCA up 11 weeks gestation. UA pulsatility index (PI) decreased with gestation (1st TP: $1.8 \pm 0.3$; 3 rd TP: $1.16 \pm 0.1 ; p<0.05)$ in contrast with an increase in the Ao PI (1st TP: 1.6 \pm 0.1 ; 3rd TP: $2.1 \pm 0.3$; $p<0.05$ ); MCA PI values remained constant during pregnancy. At the AV level, peak velocities during atrial contraction (A wave) were nearly twice as high as those during early diastolic filling ( $\mathrm{E}$ wave) in early pregnancy. However, $\mathrm{E}$ wave and $\mathrm{E} / \mathrm{A}$ ratios significantly increased from the 1st TP: $E($ tric $)=20.7 \pm 5.6 \mathrm{~cm} / \mathrm{s}$; $\mathrm{E}(\mathrm{mit})=16.1 \pm 1.9 \mathrm{~cm} / \mathrm{s} ; \mathrm{E} / \mathrm{A}($ tric $)=0.57 \pm 0.09 ; \mathrm{E} / \mathrm{A}(\mathrm{mit})=0.47 \pm 0.002$ to the 2nd TP: $E$ (tric) $=35.3 \pm 4.7 \mathrm{~cm} / \mathrm{s} ; \mathrm{E}(\mathrm{mit})=34.5 \pm 5.6 \mathrm{~cm} / \mathrm{s} ; \mathrm{E} /$ $A($ tric $)=0.7 \pm 0.03 ; E / A(m i t)=0.7 \pm 0.005$. We may conclude that the combined use of transvaginal and transabdominal Doppler is unquestonably useful for the evaluation of the fetal hemodynamics; and that the definition of normal hemodynamic parameters since early phases of pregnancy. 
P-81

Changed morphology and function in hearts with looping disturbances

Bouman HGA, Broekhuizen MLA, Baasten AMJ, Gittenberger-de Groot $A C$, Wenink $A C G$

Department of Anatomy and Embryology, Leiden, Department of Obstetrics and Gynecology, Rotterdam, The Netherlands

Exposure to the vitamin A derivative retinoic acid (RA) during early pregnancy leads to cardiac malformations. In search of an interaction of morphogenesis and hemodynamics in normally and abnormally developed hearts, chick embyros were treated with RA at stage 15 of development which resulted in hearts with an intact ventricular septum and a normal course of the subaortic outflow tract to double outlet right ventricle (DORV) with a straddling tricuspid orifice or even double inlet left ventricle. These malformations are explained by a disturbance of the looping process of the heart tube. Because of hemodynamic changes, which pointed to a diminished contraction force, quantitative morphology was carefully studied. The morphological spectrum was represented by a decrease in the right ventricular myocardial volume. Minor anomalies showed a slight volume decrease, and major anomalies showed a greater decrease. In DORV, the right ventricular myocardial volume wassignificantly smaller than in shams. In addition to this smallervolume, myofibril arrangement seemed tobedisturbed, when studied with confocal microscopy. The volume of the atrioventricular cushions showed the morphological spectrum as well. However, instead of a volume decrease as found in the ventricular myocardium, a volume increase of atrioventricular cushion tissue was apparent from the least malformed heart to DORV, the latter being significantly different. The abnormal hemodynamics can be explained by the decreased ventricular myocardial volume and by the disturbed myobril arrangement. This disarrangement could cause changed forces in the myocardial wall resulting in a looping disturbance of the heart tube. The volume increase of the atrioventricular cushions is a compensatory mechanism as a result of a changed morphology rather than a direct effect of retinoic acid treatment.

\section{P-82}

Evidence that secundum atrial septal defects can become smaller or close after the second year of life

\section{Dinarevic $S$, Carvalho IS, Shinebourne EA}

Royal Brompton Hospital, London, United Kingdom

In an eight-year-period, 66 children over two years of age, were considered to require and be suitable for closure of atrial septal defect (ASD) by a transcatheter technique. All showed right ventricular volume overload on echocardiographic assessment. As, however, safety and efficacy of devices are still being tested, the children remain untreated. This allowed evaluation of the natural history of the defects in relation to size. Mean age at time of diagnosis was 33.0土36.6 (range 2-144) months. Pts were divided into 4 groups according to size of defect: Group I ( $<5 \mathrm{~mm}) \mathrm{n}=3$, Group II $(>5-10 \mathrm{~mm}) \mathrm{n}=22$, Group III $(>10-15 \mathrm{~mm}) \mathrm{n}=30$ and Group IV $(>15-20 \mathrm{~mm}) \mathrm{n}=11$. Mean followup was 39.3 \pm 26.1 (range 4-97) months. The defect end-systolic diameter on $2 \mathrm{D}$ echocardiography correlated well with the width of the colour jet across the interatrial septum $(r=0.87, p<0.002)$. In 3 pts the defect closed, and in all groups there was a significant reduction $(p=0.0008)$ in mean size of the defect (Group I, $1.67 \mathrm{~mm}$; Group II, $2.23 \mathrm{~mm}$; Group III, $3.67 \mathrm{~mm}$; Group IV, $6.7 \mathrm{~mm}$ ). In children over 2 years of age, a secundum ASD less than $5 \mathrm{~mm}$ does not require closure and some larger defects will become significantly smaller and may not require intervention.
P-83

Development of the papillary muscles of the human mitral valveimplications for malformations

Oosthoek PW, Wenink ACG, Wisse LJ, Gittenberger-de Groot AC Department of Anatomy, Leiden University, Leiden, The Netherlands

We have studied normal human hearts ranging from 5 through 18 weeks of development, using immunohistochemistry, 3-D reconstruction methods and gross inspection. In addition we studied rat hearts by scanning electron microscopy. In the youngest hearts two prominent myocardial ridges were found at the anterior and the posterior wall of the left ventricle. These ridges are continuous with the atrial myocardium in the atrioventricular junction. The anterior and posterior ridges are continuous with each other at the apical side of the left ventricle. In between the anterior and posterior ridges, the fused atrioventricular cushions are attached, connecting these ridges at the atrioventricular side. During development, the muscular ridges lose their connection with the atrial myocardium and gradually loosen from the left ventricular wall to become freely movable papillary muscles that are only attached at their bases in the left ventricle. In the meanwhile the fused atrioventricular cushion tissue remodels into the aorric valve leaflet and chords, and only the chordal part of the cushion remains attached to the developing anterior and posterior papillary muscle. This developmental process explains many malformations of the mitral valve, e.g. left ventricular outflow tract obstruction by papillary muscles, anomalous insertion of papillary muscles into the aortic leaflet of the mitral valve, and asymmetric mitral valves - all abnormalities in which the papillary muscle remains abnormally long, comparable with the embryonic situation. Malattachment of the mitral valve is thought to be related with the primitive situation in which the primordial ridges are still part of the ventricular wall.

\section{P-84}

Evaluation of pulmonary anatomy in children with Blalock-Taussig shunt-innovative application of the internal mammary catheter Bermúdez-Cañete R, Bialkowski J, Herraız I, Acerete F, Goldstein L, Pawelec M, Szkutnik M, Kowalska M Hospital Ramón y Cajal, Madrid, Spain and the Silesian Center of Cardiology, Zabrze, Poland

In children with complex cyanotic heart defects, hemodynamic invesrigations and angiographic evaluation of pulmonary artery $(\mathrm{PA})$ are essential for diagnosis and further treatment. In 111 pts (mean age $5.3 \pm 3.8$ years) with complex heart defects and severe pulmonary stenosis or atresia and previous Blalock-Taussig (BT) shunt, a new technique of BT cannulation with the internal mammary catheter has been applied. There were 71 children with a single and 40 with double BTs. After a percutaneous puncture of the femoral artery or vein, the BT shunt was catheterized selectively with the aid of a hydrophilic Terumo guide wire and internal mammary catheter ( 4 or $5 \mathrm{Fr}$ ). The access was retrograde in 103 pts and anterograde in 8 . When it was possible, a catheter was introduced coaxially into the PA, but when it was not, an angiogram was made in the proximal end of the BT shunt. With this method we succeeded in introducing the guide wire through the BT shunt into the PA in all pts, and in $104(94 \%)$ we also managed to get the catheter into the PA to measure blood pressure and realize selective angiography. Mean pulmonary artery pressure in our series was $16 \pm 10 \mathrm{~mm} \mathrm{Hg}$. In the examined pts, peripheral pulmonary stenosis was found in $53 \mathrm{pts}(48 \%)$, either congenital (in $17 \mathrm{pts}, 15 \%$ ) or iatrogenic after BT shunt (in $36 \mathrm{pts}$, $32 \%)$. Several angiograms illustrating these findings will be presented. There were no major complications during the procedures in any pts. Interventions (occlusions or dilarations) at the BT area were simplified with this technique. In conclusion, catheterization of a BT shunt with the internal mammary catheter is a very effective and safe method for determination of the anatomy and physiology of the pulmonary arterial tree. 


\section{P-85}

Double outlet left ventricle with aortic valvular atresia-a case never described

Gouton M, Sassolas F, Bozio A

Hôpital Cardro-Vasculaire Louis Pradel, Lyon, France

We describe here a newly recognized type of double outlet left ventricle (DOLV) with aortic valvular atresia. DOLV is a very rare cardiopathy; only 119 cases are yet described in the international literature, but none with aortic valvular atresia. The diagnosis of this complex cardiopathy was made in utero at the 23 rd week of pregnancy. After birth, the diagnosis was confirmed by echocardiography and angiography. The atria and the ventricles were located normally, and the great vessels were both related with the left ventricle (LV). The right ventricle (RV) was hypoplastic, and contained no infundibulum. The pulmonary trunk (PT) overrode anteriorly a large ventricular septal defect (VSD). The aorta, which was hypoplastic, arose posteriorly to the left from the PT. The aortic valve was atresic, and aorta was perfused by a large patent ductus arteriosus. At echocardiography, there was mitral-aortic and mitral-pulmonary continuity, with bilateral absence of conus. The VSD was surgically patched; the native PT, arising from the LV, was anastomosed to the horizontal aorta; and an homograft linked the RV to the pulmonary arteries. Native ascendant aorta remained in place, and perfused retrogradely into the coronary arteries. The child died 3 days after surgery. The autopsy revealed no cardiac explanation for this death and confirmed the diagnosis that both the aortic and pulmonary valves were related to the LV, and the aortic atresic valve was in fibrous continuity with the mitral valve, so that the diagnosis of DOLV with aortic valvular atresia was made. No more than 5 cases of DOLV with pulmonary atresia were described previously, but this case of DOLV is the first with aortic valvular atresia. It can be discussed whether this cardiopathy could be called a DOLV, since the aortic valve is atresic. We think so, because, although atretic, the semilunar valves are related to the LV.

\section{P-86}

Long-term outcome of 110 neonates with critical coarctation Korbmacher B, Krogmann ON, Schmitt HH, Heusch A, Bourgeois M Departments of Pediatric Cardiology, Thoracic and Cardiovascular Surgery, Heinrich Heine University, Duisseldorf, Germany

The data of 110 (male: 64; female: 46) consecutive operated newborns were studied retrospectively. Mean weight was $3270 \mathrm{~g}$, mean age at operation was 14 days. Simple coarctation was present in 31 pts (group I), 32 pts had additional big VSD (group II) and 47 (group III) had complex heart disease. The preoperative heart catheterization revealed a gradient of $<20 \mathrm{~mm} \mathrm{Hg}$ in $35 \%,>20 \mathrm{~mm} \mathrm{Hg}$ in $51.4 \%$ and $>50 \mathrm{~mm}$ $\mathrm{Hg}$ in $12.9 \%$; the height of the gradient did not correspond with the morphologic severity of the stenosis; the systolicgradient was in group I versus groups II and III, significandly increased ( $\mathrm{p}=0.0007)$. The indication for repair was the conservatively untreatable heart insufficiency: $68 \%$ were ventilated preoperatively, $35 \%$ received catecholamines, $98 \%$ diuretics and $91.5 \%$ prostaglandins. In the vast majority $(n=95)$ of pts, resection and end-toend anastomosis were performed, in 31 cases using an absorbable suture, in 18 using a continuous suture line. Early mortality was $3.2 \%(n=1)$ in group I, $25 \%(n=8)$ in group II and $36.2 \%(n=17)$ in group III; after introducing prostaglandin $\mathrm{E}_{1}, 0 \%$ in group $\mathrm{I}, 15 \%$ in II and $25 \%$ in III. Seven pts died late because of complex heart disease. Recoarctation (gradient $>20$ $\mathrm{mm} \mathrm{Hg}$ ) developed in 9 ( 4 with hypoplastic arch, 2 after SFA) of the 77 long-term survivors; 6 of these were reoperated on, 5 without residual gradient, 1 with a gradient of $25 \mathrm{~mm} \mathrm{Hg}$ without clinical symptoms. In the last 3 pts, a balloon dilation was carried out without residual gradient. Mean follow-up time was 5 years. Sixty-three pts developed normally. 3 children are mentally and physically impaired. The survival rate is $96.7 \pm 3.3 \%$ for group I, $72.7 \pm 7.8 \%$ for II and $48.5 \pm 7.3 \%$ for III. Resection and end-to-end anastomosis using a continuous absorbable suture is the method of choice according to theoretical considerations and our experiences. The number of recoarctarions in neonatal age is relatively high; reinterventions can be done safely and successfully.
P-87

Left and right ventricular diastolic function in children with dilated cardiomyopathy

Alehan FK, Özkutlu S, Alehan D, Saraçlar M

Pediatric Cardiology Unit, Hacettepe University, Ankara, Turkey

Diastolic function, particularly in terms of right ventricular filling, has not been well established in children with dilated cardiomyopathy. We evaluated left and right ventricular diastolic filling by pulsed Doppler echocardiography in 16 children (mean age: 6.5 years) with dilated cardiomyopathy and in 20 healthy age-matched control subjects. The cardiomyopathy group demonstrated an abnormal relaxation pattern of the left ventricle. Peak early filling velocities $(43.3 \pm 11$ versus $60.4 \pm 11$ $\mathrm{cm} / \mathrm{s}, \mathrm{p}<0.005)$ and the corresponding velocity-time integrals $(3.3 \pm 1.4$ versus $4.6 \pm 1.2 \mathrm{~cm}, \mathrm{p}<0.01$ ) were significantly lower for the cardiomyopathy group. In addition, the ratio of peak early filling velocity to late filling velocity was significantly lower $(1.22 \pm 0.47$ versus $1.49 \pm 0.23$, $\mathrm{p}<0.05)$, whereas isovolumic relaxation time was significantly longer $(58.9 \pm 19.8$ versus $49.7 \pm 8.9 \mathrm{msec}, \mathrm{p}<0.05)$ in the cardiomyopathy group compared to normal subjects. Right ventricular diastolic filling was also impaired in children with dilated cardiomyopathy. Peak early filling velocities $(41 \pm 7.9$ versus $47.5 \pm 8.8 \mathrm{~cm} / \mathrm{s}, \mathrm{p}<0.05)$ and the corresponding velocity time integrals $(3.0 \pm 1.0$ versus $3.87 \pm 1.1 \mathrm{~cm}, \mathrm{p}<0.05)$ were significantly decreased, while isovolumic relaxation time was significantly increased $(60.6 \pm 16.3$ versus $52.2 \pm 12.8 \mathrm{msec}, \mathrm{p}<0.05)$ in the cardiomyopathy group. The study suggests that abnormalities of both right and left ventricular diastolic function may occur and should be searched for in patients with dilated cardiomyopathy.

\section{P-88}

Outcome of one-stage versus two-stage repair for interrupted aortic arch Bennink GBWE, Benatar AA, van de Wal HHJC

Pediatric Heart Centre, Wilhelmina Children's Hospital, Utrecht, The Netherlands

All patients with interrupted aortic arch (IAA) undergoing a one-or twostage repair were evaluated retrospectively for outcome morbidity and incidence of restenosis. From 1977 to 1995, 24 patients with IAA were diagnosed; 13 had type A, 11 type B. Twenty-two patients underwent surgical repair; two patients died prior to surgery from low cardiac output. An AVSD was present in 23 patients, aortopulmonary window in one, and additional cardiac lesions in 12 patients. Two patients had DiGeorge syndrome, and one a chromosomal anomaly. Prior to 1984 all patients had a two-stage repair (10 patients, Group I). Since 1987, a one-stage approach (12 patients, Group II) was adopted. Survival for Group I was $40 \%$ and Group II $58.3 \%(\mathrm{p}=0.32)$ Median age at correction was 64 (range 16 to 177) days for Group I and 9.5 (range 2295 ) days for Group II ( $p=0.30$ ). The majority of the Group I survivors $(75 \%)$ had their first-stage repairs before day five of life, while all Group II survivors were operated on after day five of life. In Group I, three out of four patients were left with a residual gradient at the sice of IAA repair, requiring re-operation in two and successful balloon dilatation in one. In Group II, four out of seven patients had a significant residual gradient across the aortic arch. Three underwent successful balloon dilatation and one re-operation. Mean follow-up in Group I is 125.5 months (range 94-157) and 43.4 months (range 7-119) in Group II. In conclusion, one-stage repair of IAA with associated lesions can be performed with improved survival, postoperative outcome and a trend ofdiminishing morbidity as compared to the two-stage repair. Restenosis at site of repair is amenable to balloon dilatation in most instances. 
P-89

Closure of residual leak after umbrella occlusion of the patent ductus arteriosus using Gianturco coils

de Moor M, Al Fadley F, Galal O

Department of Cardiovascular Diseases, King Faisal Specialist Hospital, Riyadh, Saudi Arabia

Residual leak after transcatheter occlusion of the patent ductus arteriosus (PDA) using the Rashkind double umbrella technique (umbrella) is a well documented problem. At our institution there is a $15 \%$ incidence of persistent residual leak after 12 months. Until September 1994 , residual leaks were occluded using a second umbrella device. The purpose of this study was to determine the results of (non-detachable) Gianturco coil occlusion of residual leak after a previous umbrella. From September 1994 until September 1995, 15 pts have undergone coil occlusion of the residual leak. The median age of the pts was 48 months (range 12 to 354 months), and the median weight was $13.6 \mathrm{~kg}$ (range $82-87 \mathrm{~kg}$ ). Seven pts had a continuous murmur and 8 had a short soft ejection murmur. Eleven pts had a previous $12 \mathrm{~mm}$ device and $4 \mathrm{pts}$ a previous $17 \mathrm{~mm}$ device. Twelve pts had the procedure on an outpatient basis. The median fluoroscopy time was 11 minutes (range 4-16 mins) The anterograde approach was used in 13 pts; the retrograde approach in 2 pts. Eleven pts required a single coil, 2 pts had 2 coils and 2 pts had 3 coils. Eleven pts had total occlusion demonstrated on angiography 510 minutes after coil occlusion. A further 3 pts were demonstrated on color Doppler echocardiography to have no residual leak. Only 1 pt had a small residual leak at the time of hospital discharge. There were no complications. There was no incidence of embolization of a coil down the aorta or the pulmonary artery. In two pts a snare was used to reposition the coil from an unsarisfactory position across the device. In conclusion, (non-detachable) Gianturco coil occlusion is very effective for residual leak on a previous umbrella. There have been no complications in this group of patients, and this is the least expensive method of dealing with residual leak.

\section{P-90}

Quantification of mitral regurgitation after repair of complete atrioventricular septal defect

Fischer G, Jung O, Stieh J, Onnasch DGW, Schmiel FK, Pollmeier A, Kramer $\mathrm{HH}$

\section{Pediatric Cardıology, Christian Albrechts Universität, Kiel, Germany}

Thirteen children ( $58 \pm 48$ months, $0.71 \pm 0.3 \mathrm{~m}^{2} \mathrm{BSA}$ ) with surgically repaired complete atrioventricular septal defect who had mitral regurgitation on auscultation, were investigated by two-dimensional echocardiography (E), Doppler echocardiography (D), angiographic (A); hemodynamic examinations were performed $46 \pm 42$ months (range 6-152 months) after correction with the aim to quantify its severity. A residual shunt did not exist; the mean pulmonary pressure $(18 \pm 4 \mathrm{~mm} \mathrm{Hg})$ and pulmonary resistance $\left(2.3 \pm 1.1 \mathrm{U} \cdot \mathrm{m}^{2}\right)$ were normal. The end-diastolic volume (A) of the left ventricle was increased 1 SD above the mean and correlated well ( $r=0.93$ ) with the end-diastolic diameter $(E)$. The stroke volume (A) was $1.94 \mathrm{ml} / \mathrm{kg}$ above the mean $(\mathrm{p}<0.05)$. The regurgitant stroke volume was ascertained by densitometry and also by subtraction of the forward stroke volume, which was determined by thermodilution, from the total stroke volume and reported as regurgitant fraction. The first method revealed $28 \pm 16 \%$ (range $6-62 \%$ ), the second one $37 \pm 14 \%$ (range 17-61\%). The correlation between both was $\mathrm{r}=0.61$. The mitralto-aortic velocity-time integral ratio (D) of $1.18 \pm 0.42$ was clearly above the mean of $0.66 \pm 0.13$ and enabled a safe identification of patients with mitral regurgitation rated by angiography as severe (IVth degree). In comparison, data ascertained by 2D-Doppler flow mapping (Mejboom er al., Circulation, 1988) correlated very unfavorably with the results achieved by angiography as well as with the end-diastolic diameter $(E)$, and the mitral-to-aortic velocity-time integral ratio. The latter parameters are therefore of greatest value for rating the severity of mitral regurgitation.
P-91

Mitral valve reconstruction and replacement in the repair of complete atrioventricular septal defects

Grabitz RG, Seghaye MC, Münnix L, Hügel W, Messmer BJ, von Bernuth $G$

Departments of Paediatric Cardiology, Thoracic and Cardiovascular Surgery, Aachen University of Technology, Aachen, Germany

The postoperative prognosis after CAVSD is largely determined by mitral insufficiency (MI) and stenosis (MS). We report 89 consecutive patients (pts) (trisomy 21: $n=64$ ) with cAVSD (type $A: n=55$; type B: $n=4 ;$ type $C: n=26$, indifferent: $n=4$; association with tetralogy of Fallot: $\mathrm{n}=5$ ) who underwent complete repair at the age of 3 to 95 (median 13) months by patch closure (single-parch: $n=76$, two-patch: $n=13$ ), AVvalve reconstruction and/or MVR. Early deaths were confined to $10 \mathrm{pts}$ (cAVSD and tetralogy of Fallot: $n=3$ ); 3 pts died during follow-up (median 2 years; 1 with severe MI, 2 with pulmonary hypertension $(P H))$. Six pts received MVR (primary: $n=2$, secondary for $M I: n=3$, secondary for MS 10 years after primary repair $n=1$ ). In 3 instances a second MVR was necessary after 18 to 120 months due to calcification or relative MS. No pt died after MVR. The remaining 76 surviving pts ( 6 with, 70 without MVR) had a median follow-up period of 4.2 years ( 3 months to 20 years). In 20 cases there was clinically no MI and at echocardiography (E) only trivial MI. These pts showed normal exercise tolerance and were without medication as were 47 further pts with clinical and E-MI, but withour left atrial (LA) enlargement. Cardiomegaly at chest $\mathrm{X}$-ray and/or LA enlargement at $\mathrm{E}$ were observed in 7 pts (on medication 4/7; NYHA 1-2). Beside a mild E-MI, we noticed a relevant subaortic stenosis in two cases (one corrected 32 months after primary repair). Only 2 pts, despite medication, were physically limited due to PH (mild MI). In our series a primary or secondary MVR was rarely necessary in the complete repair of cAVSD. A relevant but clinically fully compensated MI was present in only $7 / 76$ pts.

P-92

Effects of oxygen and inhaled nitric oxide on pulmonary resistance in children with congenital heart disease

Lunn RJ, Warner DO, O'Leary PW, Mair DM, Hagler DJ Departments of Anesthesiology and Pediatric Cardiology, Mayo Clinic, Rochester, United States of America

This study compared the effects of inhaled nitric oxide (INO) in room air (RA) and $100 \%$ oxygen $\left(\mathrm{O}_{2}\right)$ on pulmonary vascular resistance index (PVRI) in children with congenital heart disease (CHD). Studies were done during clinically indicated diagnostic catheterizations. Nine children ( 2 days-12 yrs) were enrolled and received general endotracheal anesthesia during the studies. All patients except one had pulmonary hypertension $(\mathrm{PH})$, most due to left-to-right shunts. Complete hemodynamic datasets were obtained sequentially as follows: $R A-1, O_{2}$, RA2, RA+INO (80 ppm), and RA-3. Cardiacoutput (CO) was determined by Fick method with measured $\mathrm{O}_{2}$ consumption. Results (mean $\pm \mathrm{SE}$ ):

\begin{tabular}{|c|c|c|c|c|c|c|}
\hline Condition & $\underset{1 / \mathbf{m i n} / \mathbf{m}^{2}}{\mathrm{QsI}}$ & $\begin{array}{c}\text { MAP } \\
\mathrm{mm} \mathrm{Hg}\end{array}$ & $\begin{array}{l}\text { MPAP } \\
\mathrm{mm} \mathrm{Hg}\end{array}$ & $\underset{1 / m ! n / m^{2}}{Q p l}$ & $\begin{array}{l}\text { PVRI } \\
\text { units } \mathrm{m}^{2}\end{array}$ & $\begin{array}{c}\text { SVRI } \\
\text { unitsem }{ }^{2}\end{array}$ \\
\hline RAl & $2.4 \pm 0.2$ & $54 \pm 4$ & $32 \pm 3$ & $4.6 \pm 0.8$ & $6.8 \pm 1.92$ & $21.3 \pm 2.6$ \\
\hline $100 \% \mathrm{O}_{2}$ & $2.2 \pm 0.2$ & $57 \pm 5$ & $32 \pm 4$ & $6.3 \pm 1.4^{*}$ & $47 \pm 0.97^{+}$ & $23.62 \pm 1.9$ \\
\hline RA2 & $23 \pm 0.3$ & $58 \pm 5$ & $36 \pm 4$ & $45 \pm 0.8$ & $7.96 \pm 25$ & $24.32 \pm 2.5$ \\
\hline NO 80PPM & $2.4 \pm 0.3$ & $59 \pm 6$ & $32 \pm 4$ & $7.0 \pm 15^{* *}$ & $4.19 \pm 089^{+}$ & $23.43 \pm 25$ \\
\hline RA3 & $2.5 \pm 0.3$ & $61 \pm 6$ & $35 \pm 5$ & $3.8 \pm 0.7$ & $7.96 \pm 205$ & $23.72 \pm 3.2$ \\
\hline
\end{tabular}

Both $\mathrm{O}_{2}$ and INO significantly decreased PVRI without changing MAP or systemic VRI, but QPI increased more with INO than $\mathrm{O}_{2}$. One patient with complete $A V$ septal defect responded to INO, but no change was seen with $\mathrm{O}_{2}$. In conclusion, INO and $\mathrm{O}_{2}$ are effective and selective pulmonary vasodilators in children with CHD. These data suggest that there is a greater increase in pulmonary blood flow with RA+INO than with $100 \%$ oxygen. 
P-93

Echocardiographic detection of coronary lesions in Kawasaki disease-204 patients from nationwide surveillance in Italy Pedroni E, Auriacombe L, Squarcia U, Perrone MS, Baldioli C, Klersy C, Burgio GR

Paediatric Cardiology, University of Pavia and Department of Pediatric Cardiology, Hôpital Necker-Enfants Malades, Paris, France

Toprevent coronary artery (CA) lesions and to instituteechocardiographic monitoring in KD, the Paediatric Cardiology Research Group of the Italian Paediatric Society proposed a KD Aneurysm Prevention Protocol and Nationwide Surveillance. From 1/2/88 to 31/9/95, 204 pts (136 M, $68 \mathrm{~F}$; mean age 31 months, range $2-156$ months), with clinical criteria of $\mathrm{KD}$ were submitted to the Coordinating Center in Pavia. All pts received $2 \mathrm{~g} / \mathrm{kg}$ IVIG at a mean time of 10.9 days from $\mathrm{KD}$ onset. CA evaluated on serial ECHOs showed: non-significant dilatation (diameter $\leq 1 \mathrm{~mm}$ of normal value NV) in $16 \mathrm{pts}$; abnormal diameters: aneurysm $(>1 \mathrm{~mm}$ of NV but $<8 \mathrm{~mm}$ ) in 21 pts, giant aneurysm $(\geq 8$ $\mathrm{mm}$ ) in 6 pts. Fourteen pts had multiple aneurysms. ECHO showed left ventricular dilatation in $5 \mathrm{pts}$ and decreased fractional shortening in 7; pericardial effusion was seen in 26 pts, with CA lesions in 16/26. In 2 pts (11 and 9 months old), with myocardial infarction, ECHO detected aneurysms but did not identify the fatal thrombosis (autopsy report) in the younger baby. The pts with aneurysms received IVIG later than the others (mean 13.6 vs 10.3 days). Thirty days after IVIG, ECHO followup showed persistence of aneurysms in all $27 \mathrm{pts}$. In conclusion, the significant difference $(\mathrm{p}<0.05)$ between mean infusion days in children with and without aneurysms emphasizes the crucial importance of early infusion. ECHO is a sensitive and specific test for detecting and monitoring proximal coronary aneurysms (? distal), but for the identifcation of coronary thrombosis more sophisticated diagnostic tests are necessary.

\section{P-94}

Transcatheter occlusion of the arterial duct with Jackson coilsearly experience

Gibbs JL, Uzun O, Parsons JM, Dickinson DF

Killingbeck Hospital, Leeds, United Kingdom

Percutaneous closure of the arterial duct using Jackson coils (Cook) was attempted on 26 occasions in 25 pts whose age ranged from 1 month to 67 years and whose weight ranged from $2.7-90 \mathrm{~kg}$. The diameter of the duct at its nartowest point ranged from $1-3 \mathrm{~mm}$. A single catheter technique was used in 8 cases; 6 had only right heart catheterization using a 4 or $5.5 \mathrm{Fr}$ catheter, and 2 had only arterial catheterization using a $4 \mathrm{Fr}$ catheter. In the remaining 18 procedures, left and right heart catheterization was used. Coil embolization to the pulmonary artery occurred during the procedure in 4 pts, the coil being easily retrieved in each case. On 15 occasions a single coil was implanted and on 11 occasions two coils were used. No more than two coils were implanted in any pt. On the 15 occasions with a single coil, 12 had complete occlusion by the following morning. Of the 3 with residual leaks, 1 still has a small shunt with a soft murmur 6 months later; the other 2 coils had embolized late to the left pulmonary artery. These were retrieved the next day; 1 pt had successful reocclusion with 2 coils but no further attempt to occlude the duct was made in the other case. When two coils were used $(n=11)$, complete occlusion was achieved in 9 cases. Fluoroscopy time in all the patients ranged from 3.7 to 57.5 mins. Overall, complete duct occlusion occurred in $22(88 \%)$ of the 25 patients. Patients who required 2 coils had mean minimum duct diameter of 2.8 $\mathrm{mm}$ and those with a single coil a mean diameter of $2 \mathrm{~mm}$. There was no evidence of any fow disturbance in the descending aorta or the branch pulmonary arteries following coil implantation, with follow-up ranging from 1 to 9 months. Occlusion of the duct with Jackson coils is safe. Complete occlusion is often achievable with a single coil if the duct is 2 $\mathrm{mm}$ diameter or less. The technique is at least as successful as the Rashkind umbrella and is possible even in neonates.

\section{P-95}

New possibilities in the evaluation of patients by combination of exercise testing and ambulatory blood pressure monitoring

Buss $M$, Woltersdorf V, Döhlemann $C$

Haunersche Kinderklinik, Munchen, Germany

To establish whether the combination of exercise testing and ABPM yields additional predictive values for interpreting blood pressure response in children and adolescents, we examined 52 pts with hererogeneous diseases mainly of cardiac, renal and central nervous etiology (33 boys, age 5-17 yrs, mean 11.7 yrs; 19 girls, age 5-23 yrs, mean 11,8 yrs). According to the blood pressure (BP) measured at rest and during exercise, our patients were divided into four groups with: (1) normal BP at rest and during exercise testing, $n=25 ;(2)$ normal $B P$ at rest and pathologic exercise testing, $n=8$; (3) borderline hypertension at rest and (a) normal or (b) parhologic exercise testing, $n=7$; (4) hypertension at rest and at exercise testing, $n=12$. The following characteristics could be established: Two pts of group 1 (post CoA-resection) showed normal BP at rest and in exercise testing but increased mean systolic pressures in ABPM. $6 / 8$ pts in group 2 suffering from renal diseases, diabetes or obesity responded with pathological BP during the exercise. Three of them also showed reduced circadian rhythm and/or elevated mean pressures in ABPM. Onept ingroup 3 with polycystic kidneys, although borderline hypertensive at rest, had normal BP response in exercise testing. Here ABPM revealed reduced circadian rhythm. In the pts in group $3 \mathrm{~b}$ ABPM showed abnormalities in mean pressure, systolic pressure peaks and circadian rhythm. 9/12 pts in group 4 underwent ABPM which, in each case, showed pathological BP behavior with characteristics as cited in group $3 \mathrm{~b}$. Echocardiography and ECG poorly correlated with these findings, being normal in all pts in group 3 and showing pathological results only in 6/12 pts (Echo) and 3/12 (ECG) in group 4. We conclude that exercise testing and ABPM used in combination are of higher reliability in assessing $B P$ response in chıldren and adolescents. In comparison Echo and ECG show low sensitivity.

\section{P-96}

Long-term outcome in simple transposition in western Sweden 1964-1983

Gilljam T, Eriksson BO, Solymar $L$

Pediatric Cardiology, Östra Sjukhuset, Göteborg, Sweden

In 1994 we surveyed all cases with transposition born in western Sweden 1964-1983. Of 108 pts, giving an incidence of $0.24 / 1,000$ births, 73 (68\%) were simple transpositions. All cases born in 1964 died since no palliation was attempted. From 1965 there was a gradual decrease in the preoperative mortality to $20 \%$ in $1979-83$. The hospital mortality was $30 \%$ in 27 Mustard (M) operations in $1970-78$ and $17 \%$ in 18 Senning (S) operations in 1978-84. One arterial switch was unsuccessful. Of 34 operative survivors, 10 were reoperared ( 4 baffle dysfunction, 3 baffle leaks, 1 valvular prosthesis, 3 pacemakers and 1 heart-lung transplant). Seven $M$ and $2 S$ pts died late. The causes of death were pulmonary venous obstruction (2), arrhyth mia (5), systemic ventricle dysfunction (1) and pulmonary vascular disease (1). The cumulative survival in all 73 pts was 0.43 at 5 and 10 years, 0.37 at 15 years, 0.32 at 20 years and 0.27 at 25 years of age. In 1988-91, all 28 long-term survivors after $M$ and $S$ operations undertaken in Göteborg were reinvestigated using echocardiography, catheterization with angiography and Holter monitoring. There were $13 \mathrm{M}$ pts, age 16.7 years (12.0-22.0) with a follow-up time of $14.2(11.0-18.0)$ years and $15 \mathrm{~S}$ pts age $9.4(7.2-12.1)$ years with a follow-up time of 8.7 (6.3-10.9) years. Three $M$ and one $S$ pt had caval obstruction; $2 \mathrm{M}$ and $2 \mathrm{~S}$ pts had atrial shunts; $2 \mathrm{M}$ pts had considerable pulmonary hypertension; $7 \mathrm{M}$ and $2 \mathrm{~S}$ pts had depressed systemic ventricular function and $3 \mathrm{M}$ and $6 \mathrm{~S}$ pts had dominantly junctional rhythm, tachyarrhythmais or atrioventricular block. $77 \%$ of $\mathrm{M}$ and $47 \%$ of $S$ had obvious hemodynamic aberrations or arrhythmias. In a population treated at a small centre during the evolvement of therapy, long-term survival was low due to a relatively high operative mortality and late death rate. In survivors operated on during the early period, the long-term status was less satisfactory. 
P-97

Posterolateral right thoracotomy for open heart surgery in children-a new surgical approach

Planché C, Houyel L, Petit J, Sousa-Uva M, Roussin R, Lacour-Gayet F, Serraf A

Marie-Lannelongue Hospital, Le Plessis-Robinson, France

Surgical closure of isolated cardiac defects via a median sternotomy is now performed early in life with a very low mortality and excellent longterm results. The only sequela of the operation is then the sternotomy scar, which can cause psychological prejudice, especially in girls. Between $07 / 93$ and 10/95, 57 consecutive children were operated on via a posterolateral right thoracotomy, selected on the possibility of repairing the defect by a right atrial (RA) approach exclusively. Cardiac anomalies were atrial septal defect (ASD) in 53: ostium secundum 47, ostium primum 1 , sinus venosus 5 , and perimembranous ventricular septal defect (VSD) in 4. Mean age was 6 yrs. Mean weight was $18.9 \mathrm{~kg}$. Surgery was performed with the child in left lateral decubitus. Incision was made through the 6th intercostal space, never trespassing the median axillary line. Caval and aortic cannulation were performed as usually. The RA was opened in a low position, parallel to the atrioventricular sulcus. VSD were closed through the tricuspid valve. Associated lesions were repaired in the same time in 7 pts: partial anomalous pulmonary venous return (5), valvular pulmonary stenosis (1), ductus arteriosus (1). Complete repair was achieved in all. Mean bypass time was $48.5 \mathrm{~min}$ (30-90), aortic cross-clamping being used in 8 pts. Mechanical ventilation duration was 7 hours $(0-19)$. Intensive care unit stay duration was 1.6 days (1-4). Mean duration of pleural drainage was 2 days (1-7). Transient complications were observed in 5 pts: pulmonary (3), AV block (1), LV failure (1). One pt has a residual shunt. All children are alive and well. This new approach offers considerable aesthetic advantages without additional risks in children with isolated cardiac defects curable by exclusive RA approach and applies particularly to young children and infants.

P-98

Long-term follow-up (10 to 17 years) after Mustard repair for transposition of the great arteries

Mezjboom F, Szatmari A, Utens EMWJ, Hess J

Department of Pediatrics, Division of Pediatric Cardiology and Department of Child Psychiatry, Sophia Children's Hospital, University Hospital Rotterdam, The Netherlands

Reliable numeric data concerning prevalence of late symptoms and sequelae after Mustard type repair are very scarce. Because cardiologists today face a large number of pts who underwent a Mustard or Senning type repair in the past, such numeric data are still required although the atrial switch repair is no longer treatment of choice for transposition of the great arteries. We therefore conducted a follow-up study of 91 consecutive pts who underwent a Mustard repair for transposition of the great arteries in our institution between 1973 and 1980 to assess the incidence and clinical importance of sequelae. Both mortality (24\%) and prevalence of supraventricular arrhythmia (65\%) was high in the group of pts operated upon in the years 1973-1977, but low for pts operated upon in the four subsequent years (respectively $2 \%$ and $19 \%$ ). In contrast, the incidence of baffle obstruction was substantially higher in this latter group. Right ventricular failure was not seen in any of the pts. The maximal exercise capacity of all pts was below normal (mean $84 \pm 16 \%$ ). In conclusion, this study, based on a consecutive series of pts, provides numeric data on the prevalence of sequelae after Mustard type repair performed in one hospital between 1973 and 1980. In contrast to the results in the earlier years, long-term survival is good and the prevalence of arrhythmia is low in the group of pts operated upon between 1977 and 1980. A substantially decreased exercise capacity seems to be inherent to the Mustard type repair.

\section{P-99}

Reoperation for isthmic aortic coarctation-immediate- and long-term results

Pomé G, Mauri L, Vignati G, Corato A, Austoni P, Figinı A, Pellegrinı A Pediatric Cardiology and Cardiothoracic Surgery, Niguarda Hospital, Milan, Italy

The aim of this study was to evaluate the results of reoperation for isthmic aortic coarctation (AC). Between 1971 and 1993, 519 patients (pts) with AC were operated in our center. Among these $36(7 \%)$ had isthmic aortic recoarctation. Their mean age at first intervention for native $A C$ was 8.1 yrs (2 days-33 yrs); 14 pts were less than one year old. $\mathrm{AC}$ was isolated in $26 \mathrm{pts}$ and associated with others cardiac anomalies in 10. The surgical technique employed in releaving native $A C$ were: resection and end-to-end anastomosis 16 pts, resection and interposition of prosthetic conduit $10 \mathrm{pts}$, subclavian flap 3 pts. The mean age at reoperation was of $16 \mathrm{yrs} ; 26 \mathrm{pts}$ had a recoarctation, 6 pts a residual coarctation and $4(11 \%)$ a false aneurysm. Twenty-five pts were symptomatic. Preoperatively all pts had an angiographic study. The mean interval between correction of native $A C$ and reoperation was $8 \mathrm{yrs}$ ( 1 day-23 yrs). The surgical techniques employed for reoperation were: patch enlargement $19 \mathrm{pts}$, prostheric conduit $15 \mathrm{pts}$, resection and endto-end anastomosis $2 \mathrm{pts}$. Operative mortality was of $5 \%$ ( $2 \mathrm{pts}$ ), one due to bleeding and the other due to low-output syndrome. Three pts had non-fatal intraoperative bleeding. During a mean follow-up of $10 \mathrm{yrs}(0$ 5-21 yrs), 5 prs died, 3 of associared heart anomalies, 1 suddenly and 1 due to extracardiac cause. Among survivors, 27 were asymptomatic; 21 pts were eval uated during follow-up with exercise stress test, echocardiogram and/or MNR, four pts had an aortography. The transisthmic mean gradient decreased from $51 \mathrm{~mm} \mathrm{Hg}$ preoperatively to $16 \mathrm{~mm} \mathrm{Hg}$ during follow-up. One pt was successfully operated recoarctation. In conclusion, the reoperation for isthmic $A C$ is feasible with a low mortality, intraoperative bleeding is not an infrequent complication. The long-term results are good except in pts with associated heart disease.

\section{P-100}

Transesophageal echocardiography during interventional catheterization in infancy and childhood

Lam J, van Oort AM, Tanke RB, Wiegman A, Ottenkamp J Academic Medical Centre, Amsterdam and Radboud Hospital, Nijmegen, The Netherlands

To establish the potential usefulness and limitations of transesophageal echocardiography (TEE) during interventional catheterizations in infancy and childhood, TEE was used in 42 pts during interventional catheterizations; valvuloplasty of the pulmonary valve $(n=15)$ or aorric valve $(n=1)$; angioplasty of recurrent coarctation $(n=6)$ or peripheral pulmonary stenosis $(n=3)$, placing a "double umbrella device" or coil in the arterial duct $(n=7)$, performing balloon atriotomy $(n=9)$ or "blade septostomy" ( $n=1)$. Age was $1 \mathrm{~d}-15$ yrs, weight was $3.0-55 \mathrm{~kg}$. Biplane TEE probes were available in almost all cases; 32 pts were studied with pediatric biplane probes. During pulmonary valvuloplasty the longitudinal scanning plane provided measurements of the diameter of the annulus comparable with angiography and superior to precordial imaging. In one $p t$ an associated ASD was detected and the dilatation was abandoned. After placement of umbrella devices, angiography was not necessary before release of the device limiting the radiation exposure time. During angioplasty of recurrent coarctation, TEE was not helpful. In all types of interventions, placement of guide wires and intravascular devices could be well controlled and complications could be prevented. No TEE related complications occurred. In conclusion, TEE monitoring is of value in pediatric interventional cardiology making the procedure safer. 
P-101

Does persistent left superior vena cava to coronary sinus influence the growth of the left heart in the fetus?

Macedo AJ, Oosthoek PW, Wenink ACG, Bartelings MM

Department of Anatomy, University of Leiden, Leiden, The Netherlands

Out of 99 fetal necropsy specimens, 10 were studied macroscopically because of an abnormal aortic (Ao) arch. Mean gestational age was 17 weeks (range 13-26 wks), 5/10 were male, extracardiac anomalies were present in 5/10. All had atrioventricular and ventriculoarterial concordance. A perimembranous ventricular septal defect was present in $2 / 10$. Hypoplasia of the Ao arch (3/10) and of the isthmus (7/10) was found, the mean ratio of isthmus to Ao being $41 \%$ (range 30-52\%). Although only 2 of the $89 / 99$ fetuses without Ao arch anomalies (not further studied) had persistent persistent left superior vena cava to coronary sinus (LSVC), in $7 / 10$ cases with arch anomalies, LSVC was found, with a huge coronary sinus orifice in $4 / 10$. The widened coronary sinus itself never bulged into the left atrium. None of these cases had Ao valvular of the left ventricular outflow tract was present in all. Right ventricular dominance and a small left ventricle was found after the age of 20 weeks (i.e. $2 / 10$ ). Abnormalities of the mitral valve were present in $5 / 10$, consisting of asymmetry and/or malattachment. It is concluded that hypoplastic Ao arch or isthmus in the fetus in middle pregnancy is not an isolated anomaly, but forms part of pathology of the left heart. Its association with persistent LSVC has not been described in this setting in a series this large. It is hypothesized that the increased flow from the coronary sinus orifice has changed the normal hemodynamics in the right atrium, leading to a smaller right-to-left shunt and diminished left ventricular flow. stenosis, but an abnormal left ventricular musculature with narrowing

P-103

Asymmetrical fetal hearts

Azancot A, Castella E, Khouhk E, Guirguis N, Magnier S, Casasoprana A, Blot $P$

Hôpital Robert Debré, Paris, France and Hôpital de Universidad de Coimbra, Portugal

Unbalanced size of the right and left ventricles (URV/LV) is a difficult problem in fetal cardiology. Is URV/LV due to fetal congenital heart disease (cc) or to functional transitory pathology? We present a retrospective study of 26 URV/LV sequentially followed pre- and postnatally, and the outcome and a possible strategy for neonatal management. Doppler Echocardiography (DE) confirmed the URV/LV according to gestational age (GA) and excluded complex congenital malformations. Patients were divided into 3 groups. Group I $(n=14)$, URV/LV was not usually the reason for the reference. The URV/LV appeared at 34-37 wks (wks). The surface area of the LV was normal while RV was enlarged $(>2 \mathrm{SD}$ ). An aneurysm of the foramen ovale (Afo) was noted in 4 and ductal narrowing in 1 , confirmed by postnatal examination with regression of RV dilatation. Group II indudes alargespectrum of "coarctation" $(n=12)$ and were subdivided into II a and IIb. Ila was detected at a range of 22-32 wks $(n=6)$ because of a small LV area and a large RV, and features such as flopping of the fo left-to-right or bidirectional, LV abnormal filling (e>a), retrograde ductal flow and tubular aspect of the transverse arch, in the complete form. These patients had surgery in the first days of life. IIb was detected at a range of $30 \mathrm{wks}$ to term $(\mathrm{n}=4)$ and presented an enlarged RV and a small LV in 2 cases; the other features of la were found rarely. The "coarctations" were evidenced at 2 to $4 \mathrm{wks}$. In conclusion: 1) URV/LV FH with dilated RV may be related to functional anomalies such as Afo or narrow ductus; 2) URV/LV FH occurring early in pregnancy can develop features of left heart obstruction related to severe tubular coarctation, requiring immediate management of the newborn; 3) Border-line forms may reveal the "coarctation" at 2 to 3 wks of life requiring close follow-up.

\section{P-104}

Infection and inflammatory response after homograft surgery in children Sunnegardh J, Atiq-Ahmed M, Berggren H, Gilljam T, Südow $G$ Pediatric Cardiology, Östra Sjukbuset, Göteborg, Sweden

Westudied ourclinical impression of a more intense, protracted fever reaction in children after homograft surgery compared to those with open heartsurgery without homograft insertion. Pertinent parameters in the perioperative period were studied in 50 children surviving homograft surgery in our institution and in 50 age-matched controls operated with open heart surgery without homograft insertion. The mean age $\pm S D$ was $5.2 \pm 4.9$ years; mean weight $19.7 \pm 16.7 \mathrm{~kg}$. Results are expressed in means $\pm S D$ unless otherwise indicated.

$\begin{array}{lccc}\text { Parameter } & \text { Conduit Pts } & \text { Control Pts } & \text { p-value } \\ \text { Fever }>38^{\circ} \mathrm{C} \text { (days) } & 7.6 \pm 35 & 3.5 \pm 2.9 & <0.001 \\ \text { Fever }>39^{\circ} \mathrm{C}(\text { days }) & 1.7 \pm 22 & 0.7 \pm 0.9 & <001 \\ \text { Days with CRP }>5 \mathrm{mg} / 1 & 11.0 \pm 6.5 & 6.7 \pm 3.1 & <0001 \\ \text { Days with CRP }>50 \mathrm{mg} / 1 & 5.4 \pm 65 & 2.8 \pm 2.8 & <0001 \\ \text { Blood leuokocyte count }>10 \times 10^{\circ} / 1 & 26 & 24 & \mathrm{NS} \\ \text { Days with thrombocytopenia }\left(<100,000 \times 10^{\circ} / \mathrm{l}\right) & 2.7 \pm 3.9 & 0.7 \pm 14 & <0.001 \\ \text { No. with posituve blood cultures } & 3 & 1 & \mathrm{NS} \\ \text { Days with antibiotic therapy } & 10.4 \pm 8.1 & 6.1 \pm 2.9 & <0.001 \\ \text { Extracorp. Circulation time }(\mathrm{ECC})(\mathrm{mun}) & 126 \pm 61 & 85 \pm 40 & <0.001\end{array}$

Except for a weak correlation $(r=0.3, p<0.05)$ in the conduit group between ECC-time and raised CRP levels, there was no correlation between ECCtime and the number of days with fever or raised CRP levels. The postoperative course after homograft surgery in children is to a grearer extent characterized by an inflammatory reaction as compared to the course after open heart surgery without homograft insertion. The increased inflammatory response does not seem to be caused by more frequent or serious infections after homograft surgery; an adverse reaction towards the homograft itself is the most probable cause. Intensified and prolonged antibiotic therapy may not be adequate treatment in homograft-operated patients with a prolonged febrile reaction.
Recombinant human erythropoietin in the perioperative management of pediatric open heart surgery

Stein JI, Haidvogl M, Engel A, Gombotz H, Rigler B, Dacar D, Beitzke A, Gamillscheg A, Suppan C

Departments of Pediatric Cardiology, Cardiovascular Surgery and Anesthesiology, University of Graz, Graz, Austria

To prevent the need for homologous blood transfusions due to postoperative anemia in pediatric open heart surgery, we studied the efficacy of recombinant human Erythropoietin in combined parenteral administration of iron $\left(\mathrm{Fe}^{3+}\right)$. RhuEPO $(300 \mathrm{U} / \mathrm{kg})$ was given up to $6 \mathrm{x}$ perioperatively, on days $-4,-3,-1,+1$, to all pts, on days $+3,+5$ only when $\mathrm{Hb}<12 \mathrm{~g} / \mathrm{dl}$ and/or $\mathrm{Hct}<34 \%$. The first administration was done intravenously, the following subcutaneously. Iron $\left(2.5 \mathrm{mg} / \mathrm{kg} \mathrm{Fe}^{3+}\right.$ in 50 $\mathrm{ml} 0.9 \% \mathrm{NaCl}$ ) was given intravenously at the same time. Hematologic and clinical monitoring were performed at the time of medication and at discharge. Twenty pts aged 2-12 years with congenital heart disease (17 acyanotic lesions, 3 reoperations) who underwent corrective cardiac surgery with the usual perioperative management entered the study. There were no major or unusual complications or side effects due to the study medication. Intraoperative hemodilution was possible in $18 / 20$ pts to gain 10-20 ml $/ \mathrm{kg}$ autologous blood. Preoperative $\mathrm{Hb}$ and $\mathrm{Hct}$ did not increase significantly preoperatively, decreased as expected postoperatively but was up again on day $3(10.6 \pm 1.6 \mathrm{~g} / \mathrm{dl})$ without the need for further treatment. A single blood transfusion $(5-16 \mathrm{ml} / \mathrm{kg})$ became necessary within $24 \mathrm{hrs}$ postoperatively, mainly autologous transfusions; in only 2 pts was homologous blood given. RhuEPO combined with parenteral iron administration is able to reduce homologous blood transfusions, to ease intraoperative hemodilution and to prevent excessive postoperative anemia even in pediatric open heart surgery. 


\section{P-105}

Diagnostic value of echocontrast agent SHU 454 in congenital heart disease

Baysal K, Uysal S, Çetinkaya F, Küçüködük S, Akgün N, Saraçlar M Ondokuz Mayis University School of Medicine, Samsun and Hacettepe University School of Medicine, Ankara, Turkey

The echocardiographic contrast agent SHU 154 provides microbubbles of defined size (median $3 \mu \mathrm{m}$ ) in a solution of galactose. Minute gas bubbles are known to have only a limited stability in fluids. For this reason these are absorbed in the capillaries of the lung after intravenous injection and do not reach the left side of the heart. Thus the agent can be used by peripheral venous injection for the detection of tricuspid and pulmonary valve insufficiency as well as detection of intracardiac shunts or for anatomical identification in complex congenital cardiac disease. Thirty-one patients were involved in the study (age range 2 months- 13 years). The subjects had right heart lesions such as tricuspid valve insufficiency atrial or ventricular septal defects, patency of arterial duct, tetralogy of Fallot and congenitally corrected transposition. The echocardiographic examination was performed to examine apical fourchambers, parasternal short- and long-axis views with an electronic sector scanner with $2.5,3.75$ and $5 \mathrm{MHz}$ transducers. In our study the aim was to demonstrate whether peripheral venous injection of SHU $454(0.5 \mathrm{ml} / \mathrm{kg} /, \max 10 \mathrm{ml} / \mathrm{inj}$ five times) permits a precise diagnosis. SHU 454 gave great information about the outlet of the right ventricle, pulmonary vascular structure and anatomy of coronary artery in tetralogy of Fallot and about surgical indication in ASD, VSD and silent PDA, and about valve morphology in the other anomalies. SHU 454 became evident in the left ventricle in patients with tachycardia and pulmonary hypertension. Each patient received a single injection. Five patients complained about modest feeling of warmth or cold during the injections. SHU 454 is significant enough to advance this technique not only to replace the other conventional methods of diagnosis but also to create new diagnostic capabilities.

\section{P-106}

The congenital isolated apical ventricular septal defects

Atalay S, Imamoglu A, Dilek L, Altug N, Gimis H, Özkutlu S Pediatric Cardiology Department, Ankara University, Ankara, Turkey

The diagnosis of apical ventricular septal defects (VSD) is difficult and the ratio of spontaneous closure is not well known. Our purpose is to describe echocardiographic findings and the ratio of spontaneous closure in a series of 22 children with apical VSD. Twenty-two patients were identified by color-flow imaging as having a small defect at the apical portion of the ventricular seprum. The age range was 1 day to 13 years (median age 0.53 months) All patients were asymptomatic with normal telecardiogram and electrocardiogram. Small VSDs could have been established in only 6 patients by $2-D$ echocardiography. In all cases, narrow and short colored jets were seen at the apex of the heart, distal to the moderator band. Of the 16 patients who had follow-up examinations, 3 months to 3.5 years (median duration 10.5 months), there was spontaneous closure in five (31.25\%). To our knowledge, this report is thelargest series of isolated apical VSD. We can say that although closure of apical VSD is not the rule, the prognosis is excellent.
P-107

Inhaled nitric oxide for the management of increased pulmonary vascular resistance after Fontan type operations

Gamillscheg A, Zobel G, Dacar D, Stein JI, Rödl S, Berger J, Metzler H, Beitzke A, Rigler $B$

Departments of Pediatric Cardiology, Pediatrics, Cardiovascular Surgery and Anesthesiology, Graz, Austria

A reactive increase of PVR is a major cause for morbidity and mortality in the perioperative period after Fontan-type operations. To evaluate the effects of inhaled NO on low cardiac output due to increased PVR in patients after Fontan-type procedures, 10 children aged 1.5 to 17 years $(m=6.5)$ with double inlet left ventricle $(n=4)$, double outlet right ventricle $(n=3)$, tricuspid atresia $(\mathrm{n}=2)$ and criss-cross heart $(\mathrm{n}=1)$ were treared with NO after performing a total cavopulmonary anastomosis $(n=7)$ or bidirectional Glenn anastomosis $(n=3)$. Inhaled NO was applied using a microprocessor-controlled delivery system (Pulmonox, Messer Griesheim, Austria). Inhalation of NO in a mean dose of $4.5 \pm 2.5 \mathrm{ppm}(1-10)$ was started at a CVP-LAD pressure gradient $>10 \mathrm{~mm} \mathrm{Hg}$ or at a CVP $>20 \mathrm{~mm} \mathrm{Hg}$ after failure of conventional therapy.NO wasmeasuredcontinuouslyusingthechemiluminiscencemethod. Methemoglobin levels measured 34 times a day were $1.4 \pm 0.3 \%$ (0.7-3.2).

$\begin{array}{lccc} & \text { NO off } & \text { NO on } & \text { p-value } \\ \mathrm{CVP}(\mathrm{mm} \mathrm{Hg}) & 22.8 \pm 3.2 & 19 \pm 2.4 & <0001 \\ \mathrm{LAD}(\mathrm{mm} \mathrm{Hg}) & 82 \pm 3.3 & 9.6 \pm 3.2 & 0.3 \\ \mathrm{ZVD}-\mathrm{LAD}(\mathrm{mm} \mathrm{Hg}) & 146 \pm 2.6 & 7.5 \pm 2.7 & <0.001 \\ \mathrm{MAP}(\mathrm{mm} \mathrm{Hg}) & 631 \pm 129 & 67.8 \pm 120 & <0.01 \\ \mathrm{HF}(\text { beats/mn) } & 130 \pm 19 & 131 \pm 19 & 0.4 \\ \mathrm{SaO}_{2}(\%) & 824 \pm 6.1 & 89 \pm 6.4 & <0.001 \\ \mathrm{SvO}_{2}(\%) & 63 \pm 9.2 & 70.3 \pm 8.4 & <0.015\end{array}$

No adverse effects were observed during inhaled NO therapy. One parient died because of cerebral hemorrhage. In conclusion, inhaled NO even in low doses may prove an excellent agent for the postoperative management of low cardiac output secondary to increased PVR in children who have undergone Fontan-type operations.

P-108

Kearns Sayre syndrome presenting with complete heart block Özbarlas N, Bingöl G, Altinbasak S, Kiucuikosmanoglu $O$, Aķäoren $Z$ Department of Pediatric Cardiology, Cukurova University School of Medicine, Adana, Turkey

A 13-year-old boy was admitted to the hospital because of headache, vomiting and seizures. He had developed intellectual deterioration and his school performance became poor for the last six months. He also had suffered from hearing loss for one year. His height and weight were below the 3rd percentile. On examination the pulse was 40 beats $/ \mathrm{min}$ and regular. An ECG showed the existence of complete atrioventricular block. There was no morphological abnormaliry on echocardiographic examination. At the seventh day of hospitalization, a permanent pacemaker was implanted because of frequent Adams-Stokes episodes. During the following two years he developed external ophthalmoplegia and pitosis with facial paralysis. He showed progressive ataxia with weak right step. Fundoscopic examination revealed pigmentary retinopathy. Protein level of cerebrospinal fluid was elevated $(220 \mathrm{mg} / \mathrm{dl})$. At the age of 14, he was hospitalized again because of diabetic ketoacidosis. He also had goiter with normal thyroid hormone levels. Plasma lactic acid level was normal. Ragged red fibers was seen in muscle biopsy. We concluded that Kearns-Sayre Syndromeand other mitochondrial cardiomyopathies must detected in patients with heart block with unknown etiologies. 


\section{P-109}

Clinical experience with a new steroid-eluting screw-in electrode in children

Çeliker A, Alehan D, Tokel K, Oto A, Ozme $S$ Pediatric Cardiology Unit, Hacettepe University, Ankara, Turkey

Congenital cardiac malformations and postoperativechanges in anatomy and tissue characteristics may limit available sites for lead placement in children. Active fixation of leads allow the implanter to choose the exact site where the lead should be placed. However, in some reports, active fixation pacing leads have higher thresholds, pacing and sensing. New steroid-eluting electrodes, which have been shown to have lower chronic thresholds and improved sensing, are recommended to overcome this problem. The Telectronics electrode model 033-212 (Accufx II Dec) is a bipolar ventricular active fixation polyurethane pacing lead (a porous platinum/iridium electrode) with steroid (dexamethasone sodium phosphate) eluting collar. The electrode was implanted by transvenous route in $16 \mathrm{pts}, 11$ male, mean age $8.7 \pm 4.9$ yrs. Fourteen of the pts had VVIR, 1 had DDD and 1 had VVI pacemakers. Four pts, 2 of whom had corrected transposition of the great arteries, had congenital A-V block; and 12 parients had surgical complete A-V block. Follow-ups were undertaken at 1 week, 1 month, 3 months, and 9 months after implantation. There were no lead related complications and no lead displacement. Pacing thresholds were determined at $0.5 \mathrm{msec}$ pulse durations. Impedance was assessed by telemetry.

$\begin{array}{lccccc}\text { Data } & \text { Implantation } & \text { 1 Week } & \text { 1 Month } & 3 \text { Months } & 9 \text { Months } \\ \text { Threshold }(V) & 0.52 & 047 & 0.55 & 0.48 & 0.55 \\ \text { Impedance }(\Omega) & 589 & 607 & 644 & 642 & 684\end{array}$

During long-term follow-up, the steroid-eluting screw-in electrode had stable pacing thresholds and an increase of impedance. The lower chronic threshold and higher impedance allows confident use of lower amplitude outputs from the pulse generator thus increasing longevity. This will be a useful advantage particularly in pediatric patients.

\section{P-110}

Clinical anatomy of coronary circulation anomalies in congenital malformed heart

Korneti-Pekevska K, Kargovska-Klisarova A, Nikolovski M, Duganova S Institute of Anatomy, Institute of Pathology, Medical Faculty, University of Skopje, Republic of Macedonia

The authors present a morphologic survey on the modalities and surgical anatomical significance of the coronary circulation anomalies found in 26 selected cases with congenital malformed heart out of 70 newborns and infant newborns. An inspection-dissection method for profound morphological analysis postmortem has been used. Coronary artery modalities found in 9 cases were as follows: dystopic origin of anterior descending $(\mathrm{AD})$ and circumflex $(\mathrm{Cx})$ branch from the right coronary $(\mathrm{RC})$, having an aberrant preinfundibular $(\mathrm{AD})$ or retroaortic $(\mathrm{Cx})$ course; transposed origin of the left main (LM) from the right sinus and its preinfundibular course, with an interventricular rise of $A D$ and $C x$, dystopic peritruncal course of the initial part of LM (retroaortic) and RC (preinfundibular) until entering the AV sulcuses; single coronary or one with coronary luminal fistula between $\mathrm{AD}$ and right ventricle; $\mathrm{RC}$ or LM "high take off" ascending aorta. The origin, course and development of the coronary sinus (CS) within another 11 cases have shown: left horn sinus venosus persistens continued to the $C S$ with the ostium being dilated or atretic; an "unroofed" CS (lack of wall between CS and left atrium; an extremely rare case with total anomalous pulmonary venous connection to the CS. Five cases had both arterial and CS anomalies previously described. The last (26th) case had a major coronary anomaly, i.e. pulmonary origin of the left coronary artery. This study stresses that aberrant topography of coronary circulation anomalies may complicate the reconstructive (corrective) surgery of the relevant primary cardiac malformation.
P-111

Coagulation and fibrinolytic activation in infants and children after cardiopulmonary bypass with aprotinin-a prospective study Kehl HG, Kececioglu D, Nekarda T, Vielhaber H, Asfour B, Gehrmann J, VogtJ, Nowak-Göttl $U$

Westfalische Wilhelms Universität, Münster, Germany

The aim of the study was to prospectively evaluate coagulation and fibrinolytic activation after cardiopulmonary bypass with aprotinin $(2 \cdot 17000 \mathrm{U} / \mathrm{kg})$ and to correlate these findings to the clinical outcome. Forty children aged 0.1-15 years with correction or palliation of congenital heart disease were investigated. Prothrombin fragments $\mathrm{F} 1+2\left(\mathrm{Fl}+2 ; \mathrm{nmol} / 1 \cdot 10^{-1}\right.$; Behringwerke, Germany), antithrombin IIIserinesterase-complex (ATM; ng/ml; Stago, France), D-Dimer (D-D; $\mu \mathrm{g} / \mathrm{l}$; Behringwerke), plasminogen-activator-inhibitor-1 (PAI; $\mathrm{ng} / \mathrm{ml}$ Chromogenix, Sweden), tissue-type plasminogen-activator (tPA; ng/ $\mathrm{ml} \cdot 10^{-1}$; Biopool, Sweden) and Cl-inhibitor ( $\mathrm{Cl}-\mathrm{I} \cdot 10^{-3} \mathrm{~g} / \mathrm{l}$; Behringwerke) were measured before (Preop), directly after the operation (Postop), and on postoperative days 1, 4-6, and 7-9 together with a pool of healthy children (Ref). Results are shown in the Table.

$\begin{array}{lcccccc} & \text { Preop } & \text { Postop } & \text { Day 1 } & \text { Days 4-6 } & \text { Days 7-9 } & \text { Ref } \\ \text { F1+2 } & 9 \pm 5 & 17 \pm 9 & 14 \pm 1 & 18 \pm 8 & 16 \pm 2 & 4-11 \\ \text { ATM } & 101 \pm 37 & 38 \pm 27 & 30 \pm 20 & 93 \pm 72 & 63 \pm 52 & <30 \\ \text { D-D } & 17 \pm 8 & 17 \pm 3 & 29 \pm 17 & 131 \pm 14 & 113 \pm 9 & 5-48 \\ \text { PAI } & 66 \pm 17 & 167 \pm 20 & 136 \pm 39 & 136 \pm 54 & 140 \pm 57 & <100 \\ \text { tPA } & 45 \pm 10 & 52 \pm 50 & 41 \pm 18 & 52 \pm 29 & 137 \pm 65 & <12 \\ \text { C1-I } & 263 \pm 75 & 217 \pm 21 & 252 \pm 25 & 288 \pm 99 & 288 \pm 25 & 200-350\end{array}$

D-D and PAI were clearly elevated in the postoperative period. PAI was significantly (Spearman rank: $\mathrm{p}<0.05$ ) correlated with $\mathrm{F} 1+2$ and ATM. Vascular leak syndrome was present in 8 pts; 6 of them showed high levels of PAI and low levels of C1-I and 3 died with multiple organ failure. Inconclusion, cardiopulmonary bypass operations with aprotinin in infants and children are followed by activated coagulation and fibrinolysis. Enhanced levels of PAI were often associated with secondary organ dysfunction.

\section{P-112}

Primary mitral valve prolapse syndrome in children

Krzystolik-Ladzinska J, Rokicki W, Golba E, Goc B Department of Paediatric Cardiology, Silesian Medical University, Katowice, Poland

Eighty children (50 girls and 30 boys) suffering from primary mitral valve prolapse (MVP) were studied. The age of these subjects was 5 to 18 years (mean 12.5 years). MVP was diagnosed on the basis of physical and echoca rdiographic examination. In every case, the following studies were performed: routine and 24-hour ECG (according to Holter method), echocardiography, and physical performance test (according to Bruce protocol). The cause of referring the child to a cardiologist were: cardiac murmur with/or mid-systolic click in $92 \%$, chest pain in $35 \%$, feeling of dyspnea in $10.5 \%$ (there were often more than one symptom in the same subject). Familial inheritance of MVP was suggested in over $15 \%$ of our patients. Asthenic constitution with orthopedic disorders (e.g. important scoliosis, pectus excavatum, etc.) was found in about $20 \%$ of studied children. In $25 \%$ of subjects, mitral valve leaflet inspection indicated thickening and redundancy. In $67 \%$, routine ECG showed abnormalities of cardiac rhythm, conduction or repolarization. During Holter monitoring, rhythm disturbances were detected in $25 \%$ of parients. Physical performance test results were normal in every case. 


\section{P-113}

Respiratory function tests in children with acyanotic congenital heart malformations

Mazurek B, Krzywiecki A, Rokicki W, Oklek K

Department of Paediatric Cardiology and Phthisiopneumology, Silesian Medical University, Katowice, Poland

Respiratory function tests are of particular value when applied as diagnostic procedures of cardiovascular system and congenital heart diseases. These tests allow evaluation of changes in the respiratory system according to the natural history of congenital heart diseases and to study pulmonary function after surgical correction of cardiac malformation. Thirty-four children with congenital heart disease (only left-to-right shunt) were examined in this study. Static and dynamic index of ventilation, Reff, Cst, DCOSB for CO were estimated. Examination was repeated in 8-10 months after the surgical intervention. The parameters of VC, TLC before and after operation were within the normal limits. Statistically insignificant abnormal results of dynamic indices were noted. In 13 children (38\%), slightly abnormal results of studied parameters in both examinations (before and after surgery) were observed. Significant changes in DCOSB values were noted. In 17 children, the values of this parameter were below the normal range before surgery and in 29 subjects $(85 \%)$ after the procedure. It was concluded that static and dynamic indices of ventilation were not influenced by type of congenital heart disease and size of left-to-right shunt. Low values of DCOSB might be associated with extracorporeal circulation or with other, unclear, factors.

\section{P-1 14}

Exercise testing in children with pacemakers VVIR and DDD Calzolari A, Drago F, Pastore E, Turchetta A, Ragonese P

The aim of the study was to evaluate cardiovascular response during exercise testing (ET) in children with pacemaker (PM) and to compare PM VVIR and DDD. We have examined two groups of children. Group I: 7 (3 males, 4 females), mean age 12 years (range 9-14) (4 complete atrio-ventricular block (AVB), 8 corrected great vessels transposition (CGVT)) with PM VVIR. Group II: 7 ( $6 \mathrm{M}, 1 \mathrm{~F})$, mean age 11 years (range 7-14) (3 AVB, 1 CGVT, 1 cardiomyopathy, 1 leftisomerism) with PM DDD. All children have been submitted to ET on treadmill (Bruce protocol). Parameters studied included time duration (TD), maximal heart rate (HRM), systolic blood pressure max (BPM), cardiac output at rest and at peak of exercise (CO). Results have been expressed as percentage of theoretical values obtained in a group of healthy children in the Table below; we have also calculated the percentage increase of $\mathrm{CO}$.

$\begin{array}{lcccc} & \text { TD } & \text { HRM } & \text { BPM } & \text { CO increase } \\ \text { Group I } & 77.8 \pm 7.2 & 81.7 \pm 7.9 & 89.0 \pm 4.0 & 991 \pm 50.1 \\ \text { Group II } & 67.7 \pm 12.6 & 74.8 \pm 18 & 89.5 \pm 10.0 & 64.5 \pm 47.0 \\ & \mathrm{p}=0.09 & \mathrm{p}=0.38 & \mathrm{p}=\mathbf{0 . 8 9} & \mathrm{p}=\mathbf{0 . 2 1}\end{array}$

GI had a better exercise tolerance to $\mathrm{ET}$ and increase of $\mathrm{CO}$. The results demonstrate that VVIR stimulation in children, more simple from a technical point of view, causes an optimal adaptation to exercise, and a tendency to a better increase of CO in comparison to DDD stimulation.
P-115

Growth of children after heart surgery

Buljevic AD, Malcic I, Buljevic $B$

Department of Pediatric Cardiology, University Hospital Rebro, Zagreb, Croatia

We analyzed the increase in body mass (BM) and body height $(\mathrm{BH})$ after heart surgery in the study group of 185 children (G1) with congenital heart disease (CHD). We compared BM and $\mathrm{BH}$ after surgery with the correspondent values before the surgery. The control group (G2) of 50 normal subjects in sex and age did not differ from the patients [ $p>0.05$ ]. We defined BM and BH using the percentile values of The National Center for Health Statistics of the USA(NCHS) published in 1976. The measurement was performed after surgery at an interval between 85 days-9.7 yrs (mean 3.7 yrs). The study group was divided in subgroups according to the nature of the CHD: diseases with the left-to-right shunts (G1.1), primary cyanotic diseases (G1.2) and diseases with rightor left-sided obstructive lesions (G1.3). Different influences of palliation and complete correction were also studied. Differences between groups were assessed by Student's t-test for numerical data and Chisquare test for categorical parameters. Differences were considered as significant if the error probability was equal to or less than $p<0.05$. The difference in $\mathrm{BM}$ and $\mathrm{BH}$ between the study groups before theoperation and the control group was always significant $(\mathrm{p}<0.01-0.05)$. Having compared the subgroups of patients after the surgery with the healthy children, we did not find a statistically significant difference in BM ( $p>0.05$ ). However, all three subgroups of patients were statistically significantly backward as to $\mathrm{BH}$ after the operation. The results between the sexes did not differ before or after surgery $(p>0.05)$. In the majority of patients, there was a significant increase in $\mathrm{BM}$ and $\mathrm{BH}$ after palliative operation as well as after total correction, which could be recognized by the shift to the higher percentile group. Surgically treated children achieve normal body mass, but their body height remains in the lower percentile group in comparison with healthy children.

\section{P-116}

Results of echo-stress test in children after correction of tetralogy of Fallot

Kawalec W, Kubicka K, Daszkowska J, Dluzewska J Child Health Center, Warsaw, Poland

Echo-stress test was performed in 15 children after the total correction of tetralogy of Fallot. Mean age at operation was $5.1 \pm 2.5 \mathrm{yrs}$, at the study $12.7 \pm 2.7 \mathrm{yrs}$ and $7.7 \pm 1.3 \mathrm{yrs}$ at follow-up. The stress test was performed using a bicycle in the supine position. Results are presented in the Table.

$\begin{array}{lcc} & \text { Before stress test } & \text { After stress test } \\ \text { RV-PA gradient }(\mathrm{mm} \mathrm{Hg}) & 18.1 \pm 18 & 22.7 \pm 22 \\ \text { RV-RA gradient }(\mathrm{mm} \mathrm{Hg}) & 31.3 \pm 11.5 & 37.6 \pm 9.3^{*} \\ \text { Estimated RV pressure }(\mathrm{mm} \mathrm{Hg}) & 40.7 \pm 19 & 47.5 \pm 21.3^{*} \\ \text { TV-E/A rato } & 1.1 \pm 0.4 & 1.05 \pm 0.3 \\ \text { MV-E/A ratio } & 2.08 \pm 0.63 & 1.89 \pm 0.51 \\ \text { * Statistically significant } & & \end{array}$

After the stress test, tricuspid regurgitation increased in two patients and pulmonary regurgitation in five patients. We conclude that stress tests after correction of tetralogy of Fallot increases RV pressure, but does not increase RV-PA gradient. 


\section{P-117}

Ejection fraction of right ventricle and left ventricle in long-term follow-up after Senning operation for transposition of the great arteries

Ziólkowska L, Kowalik G, Buernatowicz M, Kawalec W, Ksiazyk J, Kubicka $K$

\section{Child Health Center, Warsaw, Poland}

EFRV and EFLV were calculated by first passage radionuclide angiocardiography technique in the group of 62 pts, 3-13 yrs (mean 7 yrs) after Senning operation for TGA. The atrial correction results were good; systemic and pulmonary vein obstruction as well as interatrial gradient were observed in none of the pts. On the basis of the results of radionuclide angiocardiography there were two groups of pts. Group I $(n=53,85.5 \%)$ with normal EFRV and Group II $(n=9,14.5 \%)$ with decreased EFRV. Clinical status according to NYHA, echocardiography, ECG, 24-hr Holter, exercise test and MRI were determined for each group. Group I: mean EFRV and EFLV were $52 \pm 5 \%$ and $63 \pm 6 \%$ respectively; 48 pts were in NYHA class I and 5 pts in class II; tricuspid insufficiency (TI) was found in 10 pts (18.8\%) and severe arrhythmias (SSS in 1 and AF in 1) in 2 pts. Group II: mean EFRV and EFLV were $34 \pm 5 \%$ and $65 \pm 7 \%$ respectively (compared to Group I, the lower EFRV was significant, $\mathrm{p}<0.001$ ); 8 pts were in NYHA class $\mathrm{I}$, one pt died suddenly (probable complex ventricular arrhythmia); TI in 5 pts $(55.5 \%)$ and severe arrhythmia in 2 pts (SSS in 1 and VA in 1). We conclude that in long-term follow-up after Senning operation for TGA, there is progressive dysfunction of the systemic RV which is indicated by $\mathrm{TI}$ and decreasing EFRV.
P-119

Cardiac evaluation by multigated radionuclide ventriculography and echocardiography

Tunaoghu S, Olguntïrk R, Oguz D, Vural G, Ünlï M, Gücüyener K, Köse $G$ Gazı University Medical Faculty, Departments of Pediatric Cardiology. Neurology and Nuclear Medicine, Ankara, Turkey

Cardiomyopathy, congestive heart failure and dysrhythmias are the major cardiac causes of death in the terminal stage of muscular dystrophies diseases. A total of 29 patients with muscular dystrophy (MD) aged 1.5-17 years (mean 8.2) and 29 healthy controls aged 3-14 years (mean 8.3) were prospecrively studied to investigate their cardiac involvement, using clinical parameters and EKG, telecardiogram, Holter monitoring, left ventricular systolic and diastolic functions determined by $\mathrm{M}$-mode and pulsed Doppler echocardiography, multigated radionuclide Tc99 scanning (MUGA) and signal averaged-EKG to determine the potential value of late potentials as a preclinical marker in the diagnosis of life-threatening dysrhythmias. None of the patients had cardiac symptoms, but the fractional shortening $32.21 \pm 5.65$ vs $39.86 \pm 5.7 \%$, ejection fraction (EF) $60.71 \pm 7.51$ vs $70.73 \pm 6.39 \%$, stroke volume $40.04 \pm 21.13$ vs $49.5 \pm 14.3$, $\mathrm{E}$ (early filling velocity) $0.92 \pm 0.14$ vs $1 \pm 0.15 \mathrm{~m} / \mathrm{sec}$, E acceleration time $89.2 \pm 18.2$ vs $98.8 \pm 15.8 \mathrm{msec}$, pulmonary venous diastolic velocity $0.52 \pm 0.09$ vs $0.62 \pm 0.08 \mathrm{~m} / \mathrm{sec}$, pulmonary venous systolic velocity $0.54 \pm 0.12$ vs $0.47 \pm 0.09 \mathrm{~m} / \mathrm{sec}$, pulmonary venous systolic/diastolicvelocity ratio $1.07 \pm 0.3 \mathrm{vs} 0.77 \pm 0.17$ showed significant differences between the two groups. MUGA recordings revealed out significant differences in EF $46.4 \pm 6.2$ vs $55.4 \pm 4.5 \%$ and TES (time to end-systole) $270.1 \pm 29 \mathrm{vs} 294.1 \pm 29.7 \mathrm{msec}$ as systolic indices. There were no differences between the diastolic parameters. Early detection of cardiac involvement leading to prophylactic measures may lengthen the survival.

\section{P-120}

Postraumatic rupture of the ventricular septum in a 15 -year-old girl Erecinski J, Sabiniewicz R, Aleszewicz-Baranowska J, Chejnicki M, Dymnicka S, Anisimowicz L, Rogowski J Department of Pediatric Cardiology, Medical University of Gdansk, Gdansk, Poland

Posttraumatic rupture of the ventricular septum (traumatic VSD) following non-penetrating chest trauma is rare in children. Only 18 such cases have been reported in the medical literature. Ten of these died before or after the operation. A 15 -year-old girl was in a car accident with multiorgan trauma. A loud systolic murmur iv/vi was heard on the left sternal border, a progressive congestive heart failure was observed. Echocardiography revealed a large VSD $(2 \mathrm{~cm})$ in the middle of the ventricular septum and tricuspid insufficiency. She was successfully operated six days after the trauma. A ventricular septal defect and rupture of the papillary muscle were found. A Dacron patch was placed over the torn septum and the papillary muscle was reconstructed. After this procedure the condition of the patient improved markedly. In conclusion, surgery is the method of choice following postrraumatic large VSD and echocardiography is very useful in the diagnosis of such lesion and in the postoperative follow-up. 
P-121

Midazolam - a safe and efficient sedative for children to minimize anxiety and fear during noninvasive procedures

Ljung B, Andréasson $S$

Departments of Pediatric Clinical Physiology and Pediatric Anesthesia, Östra Sjukhuset, Göteborg, Sweden

Children's anxiety during hospital visits is often underestimated. Pediatric cardiology often implies repeated examination and it is essential to reduce the distress of the child. The technical quality of noninvasive procedures like echocardiography will be impaired if the child is unable to cooperate. In order to evaluate intranasal midazolam as a sedative during noninvasive procedures, 233 children, $0.5-13$ yrs old (mean 2.7), were given midazolam (iv formulation $5 \mathrm{mg} / \mathrm{ml}$ ) as nasal drops; 143 children, $0.5-15$ yrs old (mean 4.7) were given midazolam as nasal spray. The dosage was $0.2 \mathrm{mg} / \mathrm{kg}$ ( $\max 5 \mathrm{mg}$ ). The effect of midazolam as nasal drops was good or very good in $180(77 \%)$, sufficient in $42(18 \%)$ and not sufficient in 11 children (5\%). The results were very promising, but the administration sometimes caused problems. The effect of midazolam as nasal spray was good or very good in $124(87 \%)$, sufficient in $11(8 \%)$ and not sufficient in 8 children $(6 \%)$. In spite of lower dosage with the spray, the effect seems to be favorable, probably due to better absorption in the nasal mucosa. The time of onset was approximately seven minutes in each group. Nasal administration of midazolam is a reliable and efficient way of sedating children in a diagnostic unit. Spray administration provides considerable advantages over nasal drops.

\section{Notes}

